\title{
Computational modeling of neuronal circuits: heterogeneous connectivity and nonlinear transformation in olfactory processing
}

\author{
Dissertation \\ for the award of the degree \\ "Doctor rerum naturalium" \\ of the Georg-August-Universität Göttingen \\ within the doctoral program \\ Theoretical and Computational Neuroscience \\ of the Georg-August University School of Science (GAUSS)
}

submitted by

Wen-Chuang Chou

from Taipei, Taiwan

Göttingen 2014 


\section{Thesis Committee}

Prof. Dr. Marc Timme

Network Dynamics, Max Planck Institute for Dynamics and Self-Organization

Prof. Dr. André Fiala

Dept. of Molecular Neurobiology of Behaviour, Schwann-Schleiden Research Centre

Prof. Dr. Ulrich Parlitz

Biomedical Physics Group,

Max Planck Institute for Dynamics and Self-Organization

\section{Members of the Examination Board}

Referee: Prof. Dr. Marc Timme

Network Dynamics, Max Planck Institute for Dynamics and Self-Organization

$2^{\text {nd }}$ Referee: Prof. Dr. André Fiala

Dept. of Molecular Neurobiology of Behaviour, Schwann-Schleiden Research Centre

\section{Further members of the Examination Board}

Prof. Dr. Dr. Detlev Schild

Department of Neurophysiology and Cellular Biophysics,

Göttingen University Medical School

Prof. Dr. Florentin Wörgötter

Third Institute of Physics Biophysics, Georg-August University Göttingen

Prof. Dr. Fred Wolf

Theoretical Neurophysics, Max Planck Institute for Dynamics and Self-Organization

Date of oral examination: $7^{\text {th }}$ May, 2014 
To my great parents and my beloved wife, 



\section{Contents}

1 Introduction $\quad 1$

1.1 Sensory coding in olfactory systems . . . . . . . . . . . . . . . 1

1.2 Neurons in networks . . . . . . . . . . . . . . . 2

1.3 Thesis overview . . . . . . . . . . . . . . . . 2

2 Model of recurrent networks 5

2.1 Overview.............................. 5

2.2 The antennal lobe in Drosophila . . . . . . . . . . . . . . . . . . 5

2.2.1 Olfactory sensory neurons ............... 5

2.2.2 Projection neurons and local neurons . . . . . . . . . . . 7

2.3 The role of local neurons in recurrent networks . . . . . . . . . . . 8

2.3.1 Heterogeneous interglomerular connections . . . . . . . . . . 8

2.3.2 Lateral connections - excitatory or inhibitory? . . . . . . . . . 10

2.4 Conserved architecture between insects and vertebrates . . . . . . . . 11

2.5 Model of antennal lobe . . . . . . . . . . . . . . . . . . 12

2.5.1 Neuron spiking with the Poisson process . . . . . . . . . . . . 12

2.5.2 Conductance-Based spiking model . . . . . . . . . . . . . . . 14

2.5.3 Presynaptic inhibition . . . . . . . . . . . . . . . 16

2.6 Input-output intraglomerular function . . . . . . . . . . . . . . . 18

3 Concentration-invariant odor representations 23

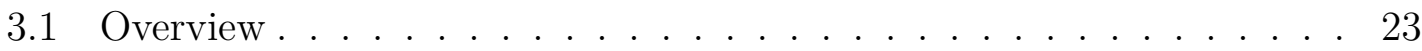

3.2 Encoding quantitative information in OSNs . . . . . . . . . . 23

3.2.1 Combinatorial coding in quantity and quality . . . . . . . . 25

3.3 Concentration-invariant coding . . . . . . . . . . . . . 26

3.3.1 Representation invariant to concentration fluctuation . . . . . 28

3.3.2 Concentration invariance within confined ranges . . . . . . . . 29

3.4 Results in theoretical investigations . . . . . . . . . . . . . . . 31

3.4 .1 Model setup . . . . . . . . . . . . . . . . . . 31

3.4.2 Experiment: single odor quality . . . . . . . . . . . . . 32

3.4.3 Weights of lateral connections . . . . . . . . . . . . . 37

3.4.4 Empirical OSN response profiles . . . . . . . . . . . . . 42

3.4.5 Experiment: two odor qualities and more inhibitory LNs . . . 47

3.5 Discussion ............................ 52

4 Discrete representations of odor mixtures $\mathbf{5 5}$

4.1 Overview . . . . . . . . . . . . . . . . . 55 
4.2 Mixture odors in olfactory processing . . . . . . . . . . . . . . 55

4.2.1 Responses of OSNs and PNs to odor mixtures . . . . . . . . 55

4.2.2 Processing odor mixtures in recurrent networks . . . . . . . . 58

4.2.3 Representations of odor mixtures . . . . . . . . . . . . 59

4.3 Results in theoretical investigations . . . . . . . . . . . . . 61

4.3.1 Model setup . . . . . . . . . . . . . . . . . . 61

4.3.2 Experiment: Odor morphing between dissimilar odors . . . . . 61

4.3.3 Experiment: Odor morphing between similar odors . . . . . . 72

4.4 Discussion . . . . . . . . . . . . . . . . . . . . . . . 78

5 Olfactory coding in modulating separation between odors 83

5.1 Overview . . . . . . . . . . . . . . . . 83

5.2 Increasing separation between representations . . . . . . . . . . . 83

5.3 Imaging data analysis . . . . . . . . . . . . . . . . . . . . . . . 85

5.4 Results in theoretical investigations . . . . . . . . . . . . . 89

5.4.1 Effects of recurrent connectivity on separability . . . . . . . 89

5.4.2 Increasing and decreasing separation in the same wiring of lateral connectivity . . . . . . . . . . . . 92

5.5 Discussion . . . . . . . . . . . . . . . . . . . . 94

6 Discussion and conclusions $\quad 99$

6.1 Discussion . . . . . . . . . . . . . . . . . . . . . 99

6.1.1 Local computation in olfactory processing . . . . . . . . . 99

6.1.2 Interglomerular interactions in olfactory circuits . . . . . . . . 100

6.1.3 More characteristics of olfactory processing . . . . . . . . . 101

6.2 Conclusions . . . . . . . . . . . . . . . . . . 102

$\begin{array}{ll}\text { Summary } & 105\end{array}$

$\begin{array}{ll}\text { Acknowledgments } & 109\end{array}$

$\begin{array}{ll}\text { Bibliography } & 111\end{array}$ 


\section{List of Figures}

2.1 Schematic diagram of the antennal lobe . . . . . . . . . . . . 6

2.2 Responses of synaptically connected OSNs and PNs in the same glomerulus. . . . . . . . . . . . . . . . . . 9

2.3 Spike trains generated by the Poisson process . . . . . . . . . . . . 13

2.4 Modeling the electrical activity of a neuron as a resistor-capacitor circuit .......................... 15

2.5 Nonlinear intraglomerular transformation and the effect of parameters 20

3.1 Responses of OSN types to distinct quality and quantity . . . . . . . 24

3.2 Discontinuous distribution of odor concentration in transmission medium ............................ 27

3.3 Concentration-invariant representations in discrete clusters . . . . . . 30

3.4 Olfactory neuron responses to odors when the strength of odor input increases linearly with concentration in logarithmic scale . . . . . . 34

3.5 Effects of increasing weights of partially-connecting local neurons . . 38

3.6 Effects of increasing weights of fully-connecting local neurons . . . . . 39

3.7 Effects of increasing weights of all local neurons . . . . . . . . . . . . 41

3.8 Olfactory neuron responses to odors when the strength of odor input is based on empirical data . . . . . . . . . . . . . . . . . 43

3.9 Improving olfactory processing by tuning lateral connectivity . . . . . 45

3.10 Weak global inhibition at high concentration helping generalization . 46

3.11 Olfactory neuron responses in networks with complicated lateral connectivity . . . . . . . . . . . . . . . . . . . . 48

3.12 Olfactory neuron responses to the second odor when the strength of odor input is based on empirical data . . . . . . . . . . . . . 49 49

3.13 The response patterns of odor $\mathrm{B}$ and odor $\mathrm{C}$ represented in the same PCA space . . . . . . . . . . . . . . . . . . 50

4.1 Definition of four interactions between constituent odors . . . . . . . 56

4.2 Clustered representations in morphing between dissimilar odors . . . 60

4.3 OSN responses to one component in different ratios in a mixture . . . 63

4.4 Olfactory neuron responses in morphing between dissimilar odors for addictive interactions in OSNs . . . . . . . . . . . . . . . . 64

4.5 Olfactory neuron responses in morphing between dissimilar odors for

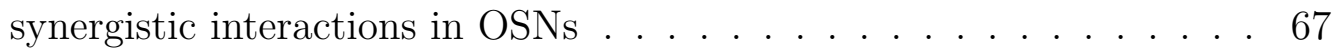

4.6 Olfactory neuron responses in morphing between dissimilar odors for hypoadditive interactions in OSNs . . . . . . . . . . . . 69 
4.7 Improving olfactory processing by tuning lateral connectivity in morphing between dissimilar odors . . . . . . . . . . . . . 70

4.8 Neurophysiological results of morphing between dissimilar odors . . . 71

4.9 Morphing between dissimilar odors based on our theoretical model . . 71

4.10 Clustered representations in morphing between similar odors . . . . . 73

4.11 Neurophysiological results of morphing between similar odors . . . . . 74

4.12 Olfactory neuron responses in morphing between similar odors for hypoadditive interactions in OSNs . . . . . . . . . . . 76

5.1 Calcium activity in the antennal lobes evoked by four odors . . . . . 85

5.2 Quantitative analysis of odor-evoked calcium activity . . . . . . . 87

5.3 Relative Euclidean distances between the calcium activity patterns of each odor pair . . . . . . . . . . . . . . . . . . 8 88

5.4 Effects of recurrent connectivity on separability . . . . . . . . . 90

5.5 Modulating the separation between odors . . . . . . . . . . . . . 93

5.6 Schematic illustration of separation and generalization in the same wiring of recurrent networks . . . . . . . . . . . . . . 96 


\section{List of Tables}

2.1 Parameters of intraglomerular transformation . . . . . . . . . . . . 19

3.1 Parameters of LNs in the antennal lobe network . . . . . . . . . . . 32

3.2 Combinatorial responses to odor A and lateral connectivity . . . . . . 33

3.3 Combinatorial responses to odor B and lateral connectivity . . . . . . 42

3.4 Combinatorial responses to odor B and C and lateral connectivity . . 47

3.5 Summary of experiments under different conditions . . . . . . . . . . 51

4.1 Combinatorial responses to odor D and E and lateral connectivity . . 62

4.2 Combinatorial responses to odor F and $\mathrm{G}$ and lateral connectivity . . 75

4.3 Summary of experiments under different conditions . . . . . . . . . . 77

5.1 Combinatorial responses to four odors and lateral connectivity . . . . 92 



\section{Introduction}

\subsection{Sensory coding in olfactory systems}

How the brain senses odors has long received attention from scientists. All the olfactory processing starts in olfactory receptors which translate odor stimuli appearing in the environment into odor signals in the form of electrical current [1]. The sensory information encoded in electrical signals is transferred between neurons as if neurons talk to each other in a forum. The study of sensory coding focuses on the relationship between external stimuli and neuronal responses in neural ensembles, and the interactions between neurons in a neuronal population or between neural ensembles across hierarchical layers.

In this thesis, we focus on how the sensory information is encoded and processed in the olfactory system. Visual information is represented on the dimension of light wavelength in the visual system, and different types of photoreceptors detect the light intensity at different wavelength bands [2]. Similarly, the sound received by ears is represented on the dimension of frequency in the auditory system where different types of hair cells sense the sound intensity at different frequency bands $[1,3]$. In contrast to these two sensory modalities, the dimension of olfactory modality is hardly described by any physical features $[4,5]$, and the odor space is constructed by different types of odorant receptors defining discrete input dimensions $[6,7]$. The number of odorant receptor types can be up to one thousand [8], resulting in extremely high dimensionality in the olfactory system as compared with any other sensory systems. Therefore, this suggests the strategies used in the olfactory modality may be different from those in other modalities.

Such distinct characteristics of olfactory processing particularly arouse our interest so that in this thesis we investigate how the olfactory system encodes and processes olfactory information represented in the sensory space with high dimensionality. The odor signals are processed in a hierarchical neural networks from peripherals to central nervous systems and are transformed several times across multiple layers [1]. How does the olfactory system exploit high-dimensional odor information across layers to integrate useful messages to identify odor quality and quantity? How do the high-dimensional signals support olfactory circuits to achieve specific olfactory strategies? Such unique olfactory processing may provide new insight into the neural mechanisms underlying sensory processing. 


\subsection{Neurons in networks}

The basic processing units in sensory systems are neurons. A neuron receives signals from other neurons via its dendrite and sends out processed signals to other neurons along its axon. A neuron can process and transmit information through electrical and chemical signals. Sensory systems implement neural ensembles consisting of large numbers of same or different types of neurons to process and represent sensory information [1]. To understand how neural ensembles work for sensory processing is a central topic in neuroscience.

The complexity of neuronal networks in terms of neuron number makes it difficult in investigating the interactions between neurons in a larger neural ensemble. It is indeed an arduous work to measure a simple relationship of neuronal responses to the input from a neighboring neuron because a neuron simultaneously interact with several neurons in networks. It is also difficult to simultaneously record the signals of all neurons in a neural ensemble because neurons are so tiny, and it is unlike to place electrodes on each neuron to measure their activity. If we can silence or activate specific neurons in networks, it could help us know local interactions in neuron pairs or specific connections.

Over the last decades, the research in neuroscience is advanced by the development of several revolutionary techniques. Optical imaging recordings can simultaneously measure the activity in large numbers of neurons $[9,10]$. Genetic tools allow experimentalist to label the neurons they are interested in [11] or to disable the functions of particular neurons [12]. In optogenetic approaches, light is used to activate or inactivate specific neurons [13]. Scientists have implemented these tools to investigate the olfactory system and gained great amount of valuable experimental data. However, there is much effort to understand how the olfactory system works based on these observations in experiments. The exact neural mechanisms underlying the olfactory processing are hitherto unknown. In this study, we will propose a theoretical model confined by biological constrains to interpret some principal functions of the olfactory system.

\subsection{Thesis overview}

Here, we make an overview of what we will study in the thesis and what we will investigate in each chapter. The reports in the field of neurophysiology, anatomy, and theoretical neuroscience studying the olfactory systems of insects as well as invertebrates will be largely cited in this study. We start with introducing the physiological properties of olfactory neurons and the connectivity of olfactory networks and proposing our mathematical model to describe the activity of neurons and neuronal interactions (Chapter 2). On the basis of this model, we explain three distinct 
phenomena in the olfactory system that have not been clearly elucidated. Each issue is discussed in individual chapters, and our main contribution in this study is composed of these three chapters.

First, we explain how the olfactory system is robust against odor concentration fluctuation and why the responses of animals to odors in different quantity but in the same quality are similar (Chapter 3). Next, we ask how odor information of multiple qualities is processed in the olfactory system and discuss why odor mixtures are perceived holistically as configural odors, not as several mixed components (Chapter 4). Third, we show that the same neural circuits can generalize different odor quality, whereas such results are distinct from the putative discrimination function in sensory processing, and ask why this kind of counterintuitive function can emerge and coexist with the discrimination function in the same wiring of connectivity (Chapter 5). Finally, we make final discussion and conclusions of our study in the olfactory system (Chapter 6). 



\section{Model of recurrent networks}

\subsection{Overview}

In this study, we focus on the first stage of odor information processing in the olfactory system: the antennal lobe in insects and the olfactory bulb in vertebrates. We first describe the physiological properties and the connectivity of olfactory neurons in the antennal lobe of insects. We also briefly talk about the architecture of the olfactory bulb and compare it with the antennal lobe. Next, we propose a mathematical model to formulate the electrical activity of different types of neurons in the antennal lobe and their interactions in recurrent networks. In this model, we demonstrate the simulation results of the input-output function in a single unit and show the properties of this function.

\subsection{The antennal lobe in Drosophila}

\subsubsection{Olfactory sensory neurons}

Three main types of neurons were discovered in the antennal lobe of an insect: OSNs (olfactory sensory neurons) at the input layer, PNs (projection neurons) at the output layer, and local neurons (LNs) [14,15] (Fig. 2.1). The odorant molecule detectors, odorant receptors, are located on the surface of the dendrites of OSNs and evoked by odorant chemicals in natural environments. The odorant receptors transfer the molecular structure of odorants into electrical signals and contribute to the activation of OSNs. In Drosophila, nearly 60 types of odorant receptors have been identified in OSNs in the antenna and maxillary palp, the olfactory sensory organs of insects [16-19]. In contrast, another species of insects, honeybees, have 160 170 types of odorant receptors [20], and in mammal, the number of odorant receptor types in mice, for example, is nearly up to one thousand [8]. Compared to approximately two million OSNs in the olfactory system of a mouse [21], Drosophila has only nearly 1300 OSNs in its antennal lobe [18,19,22-24]. The hugely reduced numerical complexity makes the investigation of the olfactory system in Drosophila easier than in other species. Therefore, many neurobiologists studying olfactory 


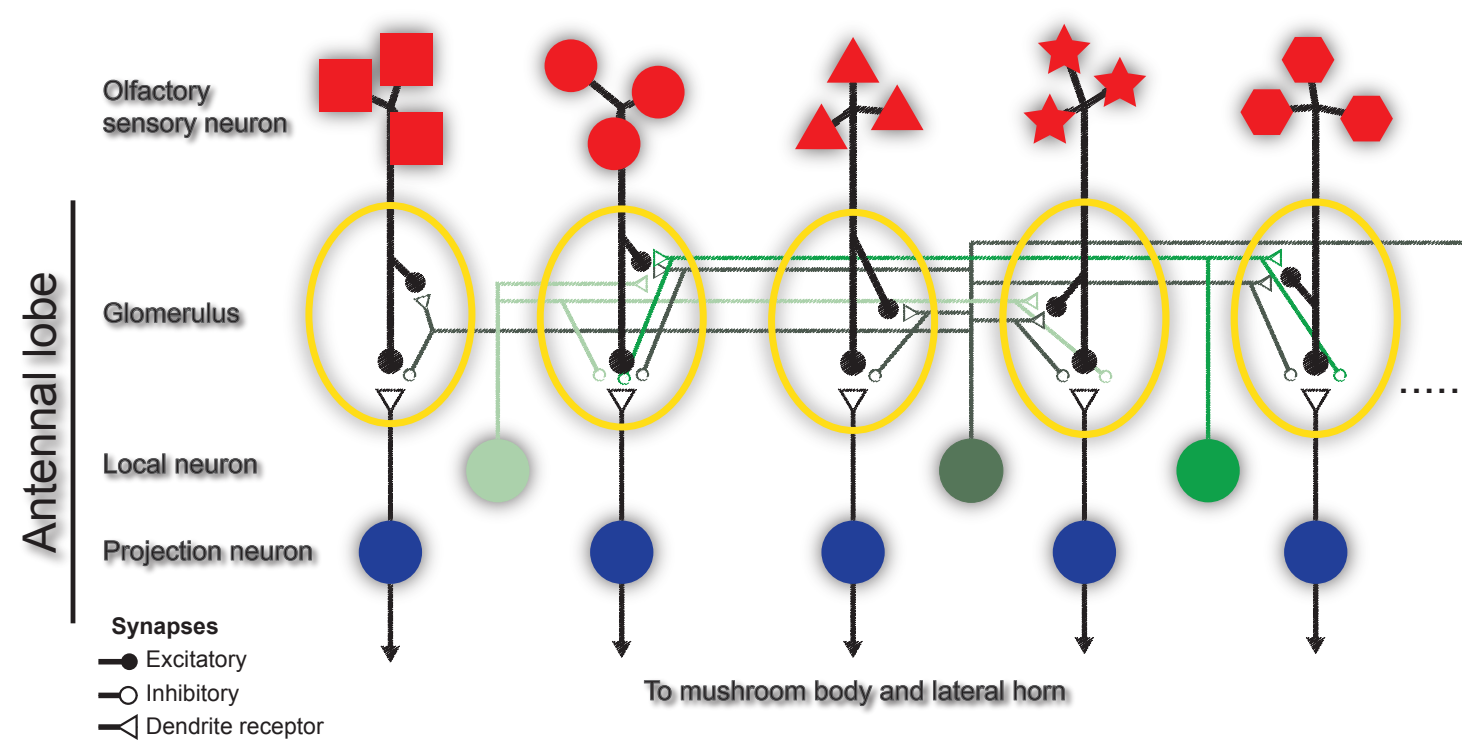

Figure 2.1: Schematic diagram of the antennal lobe. Different red symbols represent different types of olfactory sensory neurons (OSNs). The responses of different OSN types to the same odor are distinct. The same type of OSNs converges their axons to the same glomerulus. The projection neurons (PN) are the output neurons of the antennal lobe and relay the results of olfactory processing in the antennal lobe to the mushroom body and the lateral horn. PNs send their dendrites and make synaptic connections with the OSNs projecting their axons onto the same glomeruli. Local neurons (LN) laterally innervating several glomeruli receive excitatory input from OSNs and inhibit PNs in these glomeruli. Note that the connectivity of each $\mathrm{LN}$ is heterogeneous.

systems conducted experiments on Drosophila to understand the neurobiological and anatomical facts of olfactory circuits. In this thesis, we propose our model on the ground of the better-understood neural circuits of Drosophila.

A mapping in Drosophila from 45 odorant receptors to 38 OSNs was established by several researchers $[25,26]$, and it has been found that only six OSN types expressed more than one receptor [25]. The general rule for this mapping indicates one neuron expressing one receptor [27]. Interestingly, this rule also applies to mammals (mice) [28-30]. For the output of OSNs, these neurons project their axons onto parallel and stereotyped neuropil compartments called glomeruli (Fig. 2.1). The number of glomeruli in Drosophila is counted to about 50 [25,31,32]. In contrast to Drosophila, the antennal lobe in honeybees is composed of 160 170 glomeruli [33,34], and, astonishingly, the olfactory bulb in mice consists of 1600 1800 glomeruli, 35 times than the number of glomeruli in Drosophila [35].

The same OSN type expressing the same odorant receptor converges onto the same glomeruli $[25,26,30,36]$, and each glomerulus only receives the odor signals from one OSN type $[25,26]$. It has been verified the connections between 38 glomeruli and 
corresponding OSN types [25]. Some studies showed that other ten OSN types expressing ionotropic receptors $[37,38]$ are mapped to single and individual glomeruli, and only one exception, IR64a OSN type, is mapped to two glomeruli [38]. A nearly complete mapping from OSN types to glomeruli is then thoroughly explored in Drosophila.

The responses of at least 33 OSN types to more than 120 natural or artificial chemical odorants have been extensively recorded by either electrophysiological technique or calcium imaging (24 OSN types in [39], 7 OSN types in [40], and 2 OSN types in [41]). These studies suggest that each type of OSN could be activated by part of testing odorant molecules, but not by all of them, and an odorant molecule could elicit different responses for different OSN types in terms of spiking rates. Remarkably, each odorant can evoke a unique activation pattern represented by a particular combination of several OSN types. The distinct odor-evoked patterns encoding corresponding olfactory information can provide meaningful and substantial cues to downstream sensory circuits.

\subsubsection{Projection neurons and local neurons}

PNs are the output of the antennal lobe, and these neurons in each glomeruli make synaptic connections with one particular type of OSNs projecting their axons onto the same glomeruli (Fig. 2.1). In other words, PNs receive olfactory signals from their presynaptic OSNs in glomeruli where the terminals of the same OSN type converge. Because of the parallel structure of glomeruli, PNs in each glomeruli receive different odor-evoked responses from their presynaptic OSNs.

The synapses between OSNs and PNs are excitatory and very strong [12,42-45]. A report indicated that the activation of $\mathrm{PN}$ could be driven by three nearly simultaneous arrival of spikes from OSNs [45]. As the output of neural circuits, PNs relay the results of olfactory information processed in the antennal lobe to the mushroom body and the lateral horn of the protocerebrum in insects $[4,46]$.

LNs laterally innervate several glomeruli and inhibit PNs via these glomerular innervations (Fig. 2.1). The glomerular connections are reciprocal dendrodendritic synapses, meaning that the activation of LNs is dependent on the OSN responses sending out to the glomeruli where those LN lateral innervate. In other words, LNs receive odor signals from different OSN types via several glomeruli and aggregate partial odor information from specific part of glomeruli in the neural networks. The PNs are inhibited by the LNs laterally innervating glomeruli where these PNs send their dendrites. Therefore, the activation of LNs is determined by the excitatory OSN activity projected onto glomeruli where the LNs connect, and the feedback inhibition elicited by the activated LNs forms recurrent networks in the antennal lobe. Unlike the direct interactions between OSNs and PNs confined in individual 
glomeruli, LNs perform interglomerular computation collecting odor signals from multiple glomeruli and affecting the activity of PNs in several glomeruli. To summarize, LNs interact with several OSN types and the postsynaptic PNs of these OSNs in multiple glomeruli.

The inhibition from LNs onto PNs is suggested to be presynaptic, not postsynaptic $[47,48]$. The presynaptic mechanisms targeting the synapses between input neurons and output neurons were also found in the olfactory bulb of other species [49-52]. The presynaptic inhibition can modulate the strength of synapses transmitting the neuronal signals from the axons of OSNs to the dendrites of PNs. We will include such computational function in our model.

Remarkably, stronger input from OSNs does not promise stronger PN output in the same glomerulus $[12,48,53-55]$. The comparison between the responses of synaptically connected OSNs and PNs in the same glomerulus suggests that stronger input from presynaptic neurons does not elicit stronger postsynatic output (Fig. 2.2). For example, in glomeruli VM2, the OSN responses to Ethyl acetate or Ethyl butyrate are stronger than those to 1-Butanol, but the PN responses to Ethyl acetate or Ethyl butyrate are weaker than those to 1-Butanol. Because the synapses between OSNs and PNs are indeed excitatory and strong, another neuronal interactions or neural mechanisms must be involved to affect the activity of PNs. We have not talked much about the role of LNs in the neural circuits, and in the next section, we will discuss the functions and connectivity of LNs in the antennal lobe.

\subsection{The role of local neurons in recurrent networks}

\subsubsection{Heterogeneous interglomerular connections}

The LNs interconnect several glomeruli, and the interglomerular connections can be basically categorized into two types in insects. [32,56-61]. One LN type innervates all glomeruli, and the other LN type connects only particular glomeruli forming local recurrent networks $[32,56-64]$. The local connections of each LN to glomeruli are different and heterogeneous $[32,56-61,63]$. In one antennal lobe, the number of the LNs nearly connecting all glomeruli is more than the LNs locally connecting glomeruli $[32,59,61]$, and it seems that global LNs should play a role in the recurrent circuits.

Surprisingly, in the presence of odors, the LNs globally innervating glomeruli decrease their responses whereas other LNs innervating fewer glomeruli largely increase their neuronal activity [32]. These findings may suggest that global LNs are not mainly involved in the odor processing. Moreover, measuring the LN responses to odors reflected that the LN responses are odor specific $[58,63,65]$. If the LNs 


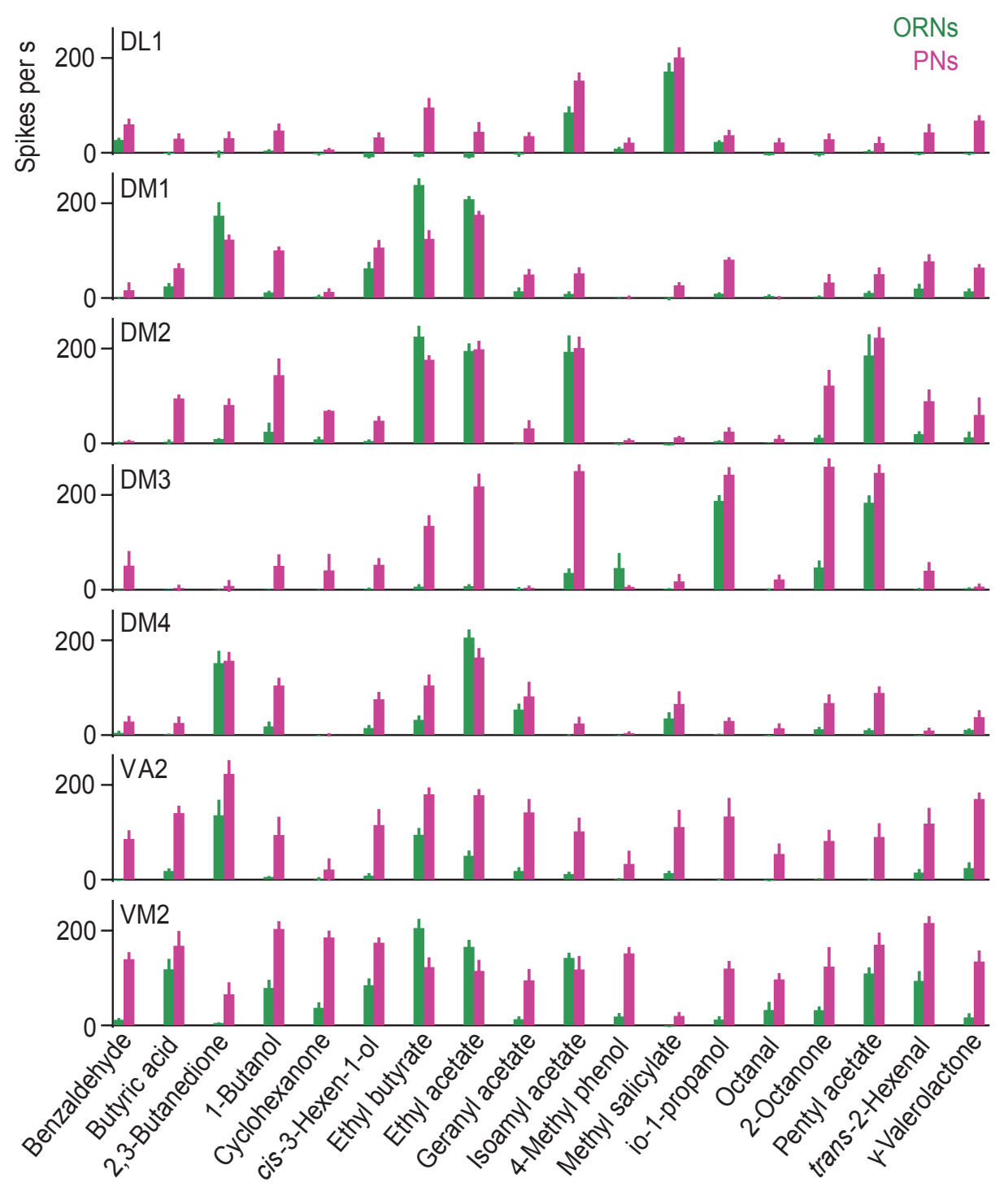

Figure 2.2: The responses of synaptically connected OSNs and PNs in the same glomerulus (green and magenta respectively). Neurons were measured in seven glomeruli in Drosophila. Different odors evoke different responses of OSNs in different glomeruli. In the same glomerulus, stronger input from OSNs does not promise stronger PN output. For example, in glomeruli VM2, the OSN responses to Ethyl acetate or Ethyl butyrate are stronger than those to 1-Butanol, but the PN responses to Ethyl acetate or Ethyl butyrate are weaker than those to 1-Butanol. Because the synapses between OSNs and PNs are excitatory and strong, another neuronal interactions or neural mechanisms must be involved to affect the activity of PNs. Reprinted by permission from Macmillan Publishers Ltd: Nature neuroscience [53], copyright (2007). 
receive neuronal signals from most of glomeruli and process odor information in the network of global connectivity, the LN should respond to most odors, not specifically to a few odors. In another study, an antagonist was used in glomeruli to suppress the reaction of inhibition receptors to the inhibition from LNs [63]. The results show that PNs in part of glomeruli increased their responses whereas the responses of PNs in other glomeruli were intact when the inhibitory pathways were interrupted. It may suggest that the effect of inhibitory LNs is glomerulus-specific, and LNs process odor signals in local recurrent networks. The glomerulus-specific was also verified by directly measuring the activity strength of LNs in several glomeruli $[58,64]$. Another recent report showed that reducing the synapses of LNs caused behavioral change on olfactory perception [66]; therefore, the activity of LNs indeed effects the odor processing in the recurrent networks of the antennal lobe.

Recent studies in the olfactory bulb of vertebrates debated whether the interactions between inhibitory neurons and output neurons are distance-dependent $[52,67,68]$ or distant-independent $[69,70]$. Notably, several works indicated that their results in computational simulations are consistent with neurophysiological data by implementing distance-independent inhibition [71,72]. Similarly, in insects, the strength of inhibition between LNs and glomeruli cannot be predicted by their spatial distances either [32, 56-61,63].

The inhibitory LNs [73] and their specific connectivity between glomeruli [74] are suggested to play an important role in olfactory processing. Therefore, we assume that the neural computation in the olfactory network mainly relies on local heterogeneous connections, not global ones.

\subsubsection{Lateral connections - excitatory or inhibitory?}

Although some experimental results indicated the lateral connections from LNs to glomeruli are excitatory $[42,43]$, another study repeating the same experiments but making minor modification showed that the lateral inhibition is the main impact in the antennal lobe [48]. The same study also reported that attenuating lateral input by removing OSNs in other glomeruli can broaden the odor tuning of PNs [48]. If lateral connections are excitatory, PNs can be activated via lateral connections by evoked OSNs in other glomeruli although the presynaptic OSNs of these PNs do not respond to the same odor. Eliminating the lateral connections will block the lateral excitation to activate PNs and make PNs narrowly tuned. Conversely, if lateral connections are inhibitory, attenuating lateral input causes PNs more easily activated by their cognate OSNs and broadens their tuning. Therefore, on the basis of their experimental results, lateral connections should be inhibitory.

A study found that the percentage of inhibited glomeruli increases as the function of odor concentration from low to intermediate dose [64,75], suggesting that increasing 
sensory input can evoke much more inhibition than excitation either from direct OSN input or lateral input until stimulus concentration reaching at intermediate level. The increasing odor concentration also results in the inhibition of a subset of PNs in specific glomeruli $[12,76]$. Moreover, the results that removal of OSNs in other glomeruli increases PN responses indicates that the lateral mechanisms should be inhibitory [48]. Another evidence showed that increasing OSN input in other glomeruli can decrease the PN responses [55]. Such inhibitory and heterogeneous architecture provide flexibility for specific interactions between parallel glomerular units in the antennal lobe [77].

\subsection{Conserved architecture between insects and vertebrates}

In the olfactory bulb of vertebrates, the output neurons mitral cells (MCs) are responsible for relaying the results of olfactory processing to higher brain areas [78]. Granule cells like LNs interconnect different MCs and mediate inhibition onto the dendrites of MCs $[79,80]$. The olfactory bulb is divided into large numbers of compartments, also called glomeruli, and these structures are the basic units in the olfactory bulb [73]. The OSNs expressing the same odorant receptor converge their axon terminals onto the same glomeruli in the olfactory bulb [81] as in the antennal lobe. The responses of MCs connecting to the same glomerulus are similar [82]. Therefore, insects and vertebrates share striking similarities in the organization of the olfactory pathways [46, 83-86].

Drosophila provides an attractive model for studying the neurophysiology of olfactory systems because of the large number of practicable genetic tools and its genome sequence being better known than other animals. Until now there has been substantial progress in understanding the anatomy and neural mechanisms underlying the olfactory processing based on the experiments in Drosophila. In this thesis, we will use these solid and widely recognized findings in Drosophila to establish our olfactory model. Most neurophysiological evidence stated in the thesis is found in Drosophila if we do not specify the animals used in the experimental studies. However, the knowledge investigating the olfactory system based on Drosophila does not mean that our results in Drosophila are not applicable to other animals. Conversely, the similar ways of processing olfactory representations and animal behavioral outputs observed in experiments across different species, coupling with the conserved olfactory architecture between insects and vertebrates suggest that the olfactory systems of animals in several phyla may share common strategies for olfactory processing [83-85].

In a recent theoretical study, a unifying network modeling the olfactory bulb and the antennal lobe was implemented to investigate the olfactory processing [87]. Here, we 
implement our model based on the neural circuits of the antennal lobe in Drosophila, but the conserved architecture between insects and vertebrates should allow us to explain common olfactory strategies.

\subsection{Model of antennal lobe}

In this thesis, we propose a mathematical model limited by biological constrains yet exhibiting the phenomena and the functions of the olfactory systems observed in recent studies. We first model the OSNs as the input of the recurrent networks and then describe the behavior of the second-order neurons PNs and other neurons LNs interconnecting glomeruli.

\subsubsection{Neuron spiking with the Poisson process}

Olfactory receptors in OSNs transfer chemical stimuli into electrical spikes. The spike rate (number of spikes per time) of an OSN indicates its preference for odor stimuli and the strength of neuronal activation. Such resulting spike rates (or called firing rates) to different odor stimuli were systematic recorded in neurophysiological experiments and published in many reports $[12,39,53,88-90]$. Once knowing each firing rate of different OSN types responding to different odors, we can then use these data as the inputs in the networks of the antennal lobe in future experimental and theoretical research.

A simple idea for simulating the neuron spiking for a given spike rate is to emit a spike periodically with constant inter-spike intervals. For example, if the spike rate of a neuron responding to an odor is set at $50 \mathrm{~Hz}$, we can represent this neuron that emits a spike every $20 \mathrm{~ms}$. However, the spiking of neurons is not normally periodic but always noisy, and they actually behave in a stochastic way. Here, we express a spike event as $x(t),\{x(t): x=\{0,1\}, t \in[0, \infty)\}$ assuming that $x(t)=1$ means a spike being emitted at time $t$, and $x(t)=0$ means no spike at time $t$. Next, we want to know the probability of emitting one or several spikes $n$ at a time interval $\Delta t$, and this probability can be denoted by $P\left(\sum_{t}^{t+\Delta t} x(t)=1\right.$ or $\left.n\right)$. To obtain this probability, we divide the time interval $\Delta t$ equally into enough small $k$ sub-intervals where at most one spike occurs and assume that the probability that one spike occurs during this sub-interval equals $p$. This is a typical form of a Bernoulli trial with exactly two possible outcomes: one spike or no spike. Therefore, the probability of $n$ spikes at a time interval $\Delta t$ can be given by the following equation:

$$
p\left(\left(\sum_{i=t}^{t+\Delta t} x(i)\right)=n\right)=\frac{k !}{n !(k-n) !} p^{n}(1-p)^{k-n} .
$$



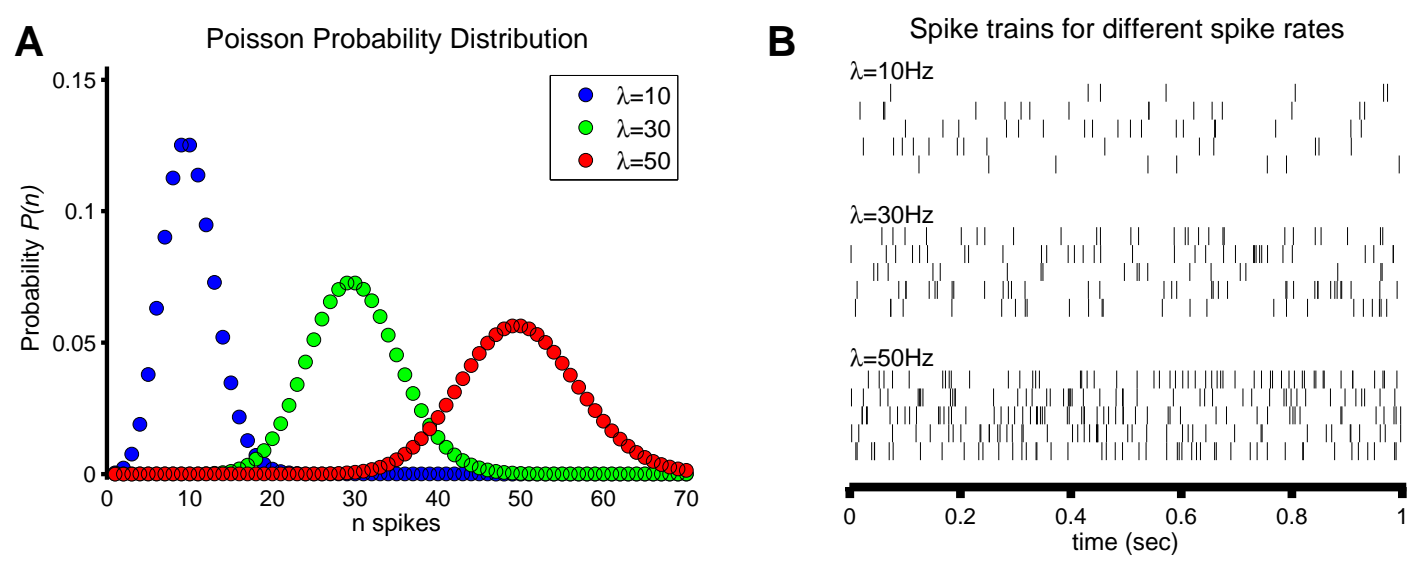

Figure 2.3: A. The probability mass function of Poisson distribution given different spike rates $\lambda$. Different colors represent the distribution with different spike rates. The highest probability always occurs at the spike rate. B. Spike trains generated by the Poisson process in different spike rates. Each row is one trial base on the given spike rate, and each spike rate runs for five times. The small vertical lines indicate spikes emitted by a neuron. Although the spike rate is identical, stochastic Poisson process generates these spike trains with great variability.

If we get the firing rate $\lambda$ from experiments, the probability $p$ of emitting a spike in a sub-interval can be replaced by $\frac{\lambda \Delta t}{k}$. Next, we further divide the time interval $\Delta t$ into an infinite number of intervals, and let $k$ be approached to $\infty$, the Eq. 2.1 can be simplified into:

$$
P(n ; \lambda \Delta t)=e^{-\lambda \Delta t} \frac{(\lambda \Delta t)^{n}}{n !}
$$

The probability distribution of Eq. 2.2 is known as Poisson distribution, and the stochastic process we described above is named as homogeneous Poisson process [91]. When we observe a neuron response in one second $(\Delta t=1 \mathrm{~s})$, the mass probability distributions as a function of spikes in one second at different firing rates are shown in Fig. 2.3A. After knowing the probability function of generating $n$ spikes within a time interval $\Delta t$, we can then numerically generate spike trains. We demonstrate simulations of generating spike trains using different firing rates, and in each different rate, the spike trains are generated for five times in Fig. 2.3B. In simulation results, each bar indicates a spike emitted by a neuron, and each row represents one trial. The higher the firing rate, the more spikes the neuron will emit. Specifically, given the same firing rate neurons will not generate exactly the same spike pattern because of the stochasticity of neurons. The source of neuronal variability or noises in this study only comes from this stochastic Poisson process.

The timing of the ith OSN spikes generated by the stochastic process is represented by a series of number $T_{i}^{s p}=\left\{T_{i, 1}^{s p}, T_{i, 2}^{s p}, \ldots, T_{i, u}^{s p}, \ldots, T_{i, k}^{s p}\right\}$. The voltage trace or action 
potential of the $i$ th OSN can be formulated by:

$$
V_{i}^{O S N}(t)=V_{0}^{O S N}+\sum_{k} \alpha\left(t-T_{i, k}^{s p}\right) \Delta V
$$

where $\alpha(n)$ is a unit function:

$$
\alpha(n)= \begin{cases}1, & \text { if } n=0 \\ 0, & \text { if } n \neq 0\end{cases}
$$

if we implement neuron activity in a discrete simulation. Here, $\Delta V$ indicates the Voltage increase when a spike is emitted by the $i$ th OSN, and $V_{0}^{O S N}$ represents the resting voltage $[92,93]$.

OSN activity modeled with Poisson statistics allows us to use firing rates elicited by specific odors recording in experimental data to generate spike trains, and these spike trains are then turned into the input to the antennal lobe. This Poisson process is commonly utilized in investigating neural network dynamics, and it has been likewise applied to model OSN activity as the input to olfactory systems in several reports [94-96].

\subsubsection{Conductance-Based spiking model}

After formulating the spiking behavior of OSNs, we make use of fundamental knowledge of electrical circuits to describe the neuronal electrical activity of PNs and LNs in this section.

Neurons are surrounded by a huge number of ions, and the variation of ions along the membrane or through the membrane channels causes the current flow. The current flow can be driven by the electrical potential difference initiated by an external input such as presynaptic current or spontaneous neuronal activation. First, we suppose a neuron acts as a capacitor and is being charged from an external current passing into it. If we set the neuron membrane capacitance $C_{m}$, membrane electrical potential (or voltage) $V(t)$, and an external current $I_{\text {ext }}(t)$, we can get a basic equation determining the voltage of this neuron as:

$$
C_{m} \frac{d V(t)}{d t}=I_{\text {ext }}(t)
$$

In this first order differential equation, the presence of external currents will charge the neuron and increase the electrical potential of neuron membrane. However, it is unlikely that the neuron capacitor boundlessly holds all electrical charge and retains the potential difference between two sides of membrane without losing any 


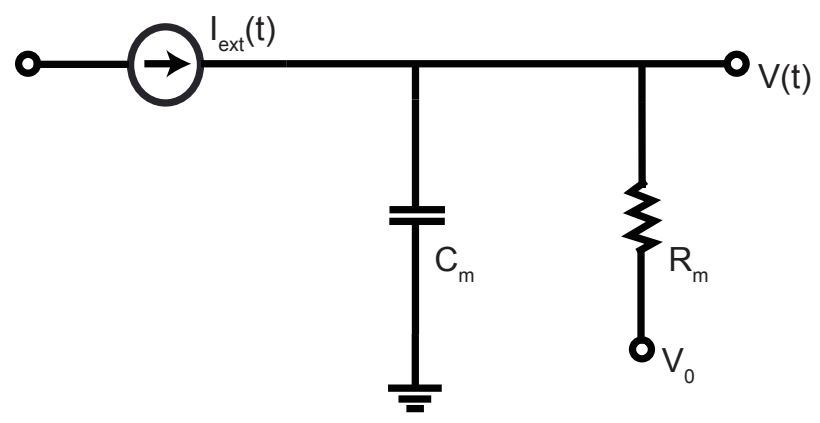

Figure 2.4: The electrical activity of a neuron can be interpreted by a resistor-capacitor coupling circuit. The membrane electrical potential $V(t)$ is driven by an external current $I_{e x t}(t)$ from synapses. Two electrophysiological parameters $R_{m}$ and $C_{m}$ are the membrane resistance and the membrane capacitance of this neuron respectively. $V_{0}$ is the resting potential of membrane. If the input current $I_{\text {ext }}(t)$ disappears, $V(t)$ will eventually return to the baseline $V_{0}$.

charge to the places at a lower electric potential. A more realistic way to express the neuronal dynamic is a resistor-capacitor circuit ( $\mathrm{RC}$ circuit) shown in Fig. 2.4. Then we have the expression of neuronal voltage based on Kirchhoff's current law in this RC circuit:

$$
C_{m} \frac{d V(t)}{d t}=-\frac{\left(V(t)-V_{0}\right)}{R_{m}}+I_{e x t}(t)
$$

where $R_{m}$ and $V_{0}$ are the resistance and the resting potential of neuron membrane respectively. Next, if we multiple membrane resistance $R_{m}$ on both sides of Eq. 2.5 and define $R_{m} C_{m}$ as membrane time constant $\tau_{m}$, the equation is then rewritten as:

$$
\tau_{m} \frac{d V(t)}{d t}=-V(t)+V_{0}+R_{m} I_{e x t}(t)
$$

Eq. 2.6 is commonly named as leaky integrate-and-fire model [92,93,97]. The leaky current results from the term $-V(t)+V_{0}$ meaning that the neuron capacitor will lose electrical charge when there is no or not enough external current to support the potential difference. When it occurs, the membrane potential will exponentially drop to the resting potential $V_{0}$. The decreasing rate is decided by the membrane time constant, and it takes $\tau_{m}$ to drop the voltage from $V\left(t^{\prime}\right)$ at time $t^{\prime}$ to $\frac{V\left(t^{\prime}\right)}{e}(e$ is Euler's number).

However, Eq. 2.6 cannot explicitly display all neuronal dynamics. When an external current causes a neuron to depolarize and to reach the threshold potential $V_{t h}$, the neuron will emit a spike. After reaching the threshold, the membrane potential will not adhere to Eq. 2.6. The potential abruptly surges up and quickly plunges to the resetting potential $V_{\text {reset }}$ below the resting potential. The probability of spiking dramatically decreases to zero for a short period because neurons cannot densely emit several spikes in a few milliseconds. This inactive period is called absolute 
refractory period $t_{r}$. After the absolute refractory period, neurons are available to emit the next spike, and its potential begins from resetting potential and again follows the leaky integrate-and-fire equation (Eq 2.6).

In the antennal lobe, LNs receive the external current from OSN axons innervating glomeruli where those LNs laterally connect, and so the amplitude of the external current to LNs results from the spike OSNs generate. Assuming that the strength of the postsynaptic response to each spike is identical, the magnitude of $I_{\text {ext }}(t)$ equals a constant value $I_{i}^{O S N}$ when an LN receives a spike from the $i$ th OSN. For a short period, the LN membrane $R_{m, L N}$ barely changes; therefore, we get a constant value $R_{m, L N} I_{i}^{O S N}$ to express the external input term in Eq. 2.6 when the $i$ th OSN emits a spike at $t=T_{i, k}^{s p}$. In addition, the external input $I_{e x t}(t)$ becomes zero when no spike occurs. The lateral connectivity of the $l$ th $\mathrm{LN}$ is represented as $w_{\kappa \rightarrow l}$, the weight of the lateral connection to the $\kappa$ th glomerulus where the $i$ th OSN projects. This arrow in $w_{\kappa \rightarrow l}$ indicates that OSN signals are transmitted from the $\kappa$ th glomerulus to the $l$ th LN. All these features characterize the membrane potential $V_{L N}(t)$ of this lth LN modified from Eq. 2.6:

$$
\tau_{m, L N} \frac{d V_{L N}(t)}{d t}=-V_{L N}(t)+V_{0, L N}+\sum_{i} w_{\kappa \rightarrow l} R_{m, L N} I_{e x t}(t)
$$

where $\tau_{m, L N}$ and $V_{0, L N}$ are the membrane time constant and the resting potential of the $l$ th LN respectively [92]. Only the $i$ th OSN projects its axons to the $\kappa$ th glomeruli where the $l$ th LN laterally innervates can send out encoding odor information to this $\mathrm{LN}$ in the form of spike trains.

The PN dynamics is more complicated than LN's because PNs receive both excitatory input from OSNs and inhibitory input from LNs. In Eq. 2.6, we replace the input term with a synaptic current $I_{s y n}(t)$ representing the interaction between excitatory OSN input and presynaptic inhibition from LNs. If we define $\tau_{m, P N}$ and $V_{0, P N}$ are the membrane time constant and the resting potential of a $\mathrm{PN}$ respectively, then the membrane potential $V_{P N}(t)$ of a $\mathrm{PN}$ is cast into the form:

$$
\tau_{m, P N} \frac{d V_{P N}(t)}{d t}=-V_{P N}(t)+V_{0, P N}+I_{s y n}(t)
$$

$[87,94]$. The input term $I_{\text {syn }}(t)$ incorporates the constant $R_{m}$ in Eq. 2.6 into a constant inside $I_{\text {syn }}(t)$ and will be interpreted in detail in the next subsection.

\subsubsection{Presynaptic inhibition}

In a glomerulus, the dendrites of PNs make a synaptic connection with the axons of OSNs. The reciprocal dendrodendritic synapses from LNs target onto the synapses 
between OSNs and PNs and apply presynaptic inhibition to the OSN-PN connections. The strength of feedback inhibition in the antennal lobe is related to the LN potential governed by Eq. 2.7. The LN equation Eq. 2.7 characterizes a strong association between the inhibition strength and OSN input from several glomeruli. Therefore, via the recurrent connections, the PN responses are affected not only by the presence of excitatory OSN spikes in the same glomeruli but also by the inhibition activated by OSN input from multiple glomeruli.

First, we model the synaptic current $I_{\text {syn }}(t)$ with a conductance $g_{i}^{\text {syn }}$ of synapses between the PN and the $i$ th OSN in the same glomerulus through the equation:

$$
I_{s y n}(t)=\sum_{i} g_{i}^{s y n}\left(V_{i}^{O S N}(t)-V_{E, s y n}\right)
$$

where $V_{E, \text { syn }}$ is the reversal potential of synapses [94,98]. If we assume the resting voltage of OSNs $V_{0}^{O S N}$ is equal to the reversal potential of synapses $V_{E, \text { syn }}$, the Eq. 2.9 can be reduced by substituting $V_{i}^{O S N}(t)$ with Eq. 2.3 and expressed as follows:

$$
I_{s y n}(t)=\sum_{i} \sum_{k} g_{i}^{s y n}(t) \alpha\left(t-T_{i, k}^{s p}\right) \Delta V .
$$

[95]. Therefore, each spike from the $i$ th OSN can generate a synaptic current to stimulate its postsynaptic PNs and to initiate PN spiking.

Moreover, the spike from $i$ th OSN to its postsynaptic PNs can increase synaptic conductance $g_{i}^{\text {syn }}(t)$ by $N_{i}^{\text {syn }}(t) p_{i}^{\text {rel }}(t) q$. Here, $N_{i}^{\text {syn }}(t)$ is the number of releasable vesicles, $p_{i}^{r e l}(t)$ is the probability of vesicular release, and q is a quantal size representing the postsynaptic response to a vesicle $[92,94,99,100]$. Because this spike uses releasable vesicles to generate a synaptic current cross the synaptic cleft, the amount of releasable vesicles $N_{i}^{s y n}(t)$ is decreased by $N_{i}^{s y n}(t) p_{i}^{r e l}(t)$.

Conversely, when the $i$ th OSN is silent, the $g_{i}^{s y n}(t)$ decays exponentially with time constant $\tau_{g}$ following the equation:

$$
\frac{d g_{i}^{s y n}(t)}{d t}=-\tau_{g} g_{i}^{s y n}(t)
$$

$[92,94,95]$. At the same time, the synapse repackages releasable vesicles for the next release, and this mechanism can be represented by an exponential recovery with another time constant $\tau_{N}$ and a maximal number of releasable vesicles $N_{0}$ :

$$
\frac{d N_{i}^{s y n}(t)}{d t}=\tau_{N}\left(N_{0}-N_{i}^{s y n}(t)\right)
$$

[94].

The presence of spikes can increase the conductance of synapses but has to spend some vesicles transmitting neural signals. As a result, the descending number of 
available synaptic vesicles will decrease the incremental amount of synaptic conductance resulting from the next spike for repetitive spiking. In contrast, although the absence of arrival spikes reduces the synaptic conductance, the synapse regains releasable vesicles at the axon terminal due to inactive neural transmission.

Next, the lateral inhibition is represented by decreasing the release probability exponentially proportional to the summation of spike rates of all LNs innervating the $\kappa$ th glomerulus. Because the lateral connections are via reciprocal dendrodendritic synapses $[15,101]$, these synapses transmit both feedforward signals from glomeruli to LNs and feedback signals in the opposite direction. In other words, mediating signals by the same synapses can allow us to assume that the weights of feedback connections $w_{l \rightarrow \kappa}$ are the same as the weights of feedforward connections $w_{k \rightarrow l}$. Then the release probability $p_{i}^{r e l}(t)$ of a synapse from the $i$ th OSN in the $\kappa$ th glomerulus is governed by the equation of an exponential form:

$$
p_{i}^{r e l}(t)=P_{\max } \exp \left(-\alpha \sum_{l} w_{l \rightarrow \kappa} f_{l, L N}(t)\right)
$$

where $P_{\max }$ is the maximum release probability, $f_{l, L N}(t)$ is the firing rate of the $l$ th LN innervating the $k$ th glomerulus, and $\alpha$ is a constant [94]. In this case, if presynaptic inhibition is applied to the synapse, the decrease of the release probability will follow the exponentially decreasing function (Eq. 2.13) and result in attenuating the increase of synaptic conductance initiated by input spikes from OSNs.

In the absence of spikes from OSNs, the synaptic conductance will keep decaying exponentially. Therefore, the extremely low synaptic conductance implies the low probability of postsynaptic PN spiking.

\subsection{Input-output intraglomerular function}

Before coming to the part of explaining how the olfactory system performs the observed phenomena, we start with looking into the relationship between OSN responses and their postsynaptic PN responses in the networks and leave aside lateral inhibition. Several recent reports indicated that the synapse between OSNs and $\mathrm{PNs}$ is very strong and can vigorously amplify weak OSN responses $[12,44,53]$. One reason of the strong connections is that glomeruli connect several dozens of OSNs with a few PNs $[19,23]$. Even though the spike rates of OSNs responding to an odor is low (e.g. 10Hz), the total spike number of an OSN population transmitted to their postsynaptic PNs can be hundreds of spikes per second. With this highconverged connectivity the odor weakly activating OSNs is still capable of causing their postsynaptic PNs to spike.

Another reason explaining this input-output amplification is likely the high release probability of vesicles at the synapses between OSNs and PNs. A recent study [44] 
Table 2.1: Parameters of intraglomerular transformation.

\section{A. PN}

\begin{tabular}{lll} 
Name & Value & Description \\
\hline \hline$\tau_{m, P N}$ & $5 \mathrm{~ms}$ & membrane time constant \\
$V_{0, P N}$ & $-60 \mathrm{mV}$ & resting potential \\
$V_{r e s e t, P N}$ & $-80 \mathrm{mV}$ & resetting potential \\
$V_{t h, P N}$ & $-45 \mathrm{mV}$ & threshold potential \\
$t_{r, P N}$ & $1 \mathrm{~ms}$ & absolute refractory period \\
\hline
\end{tabular}

\section{B. Synapse}

\begin{tabular}{lll} 
Name & Value & Description \\
\hline \hline$\Delta V$ & $8 \mathrm{mV}$ & potential increase evoked by a neuron spike \\
$\tau_{g}$ & $2 \mathrm{~ms}$ & conductance decay time constant \\
$\tau_{N}$ & $100 \mathrm{~ms}$ & vesicle recovery time constant \\
$q$ & 1.07 & quantal size \\
$N_{0}$ & 51 & maximal number of releasable vesicles \\
$P_{\max }$ & 0.79 & maximum release probability \\
$\alpha$ & 0.032 & constant for lateral inhibition \\
\hline
\end{tabular}

estimating this probability suggested that this value $P_{\max }$ could be up to 0.79 in Drosophila, and the number of release sites was estimated to be about 51. Another study also confirmed this unusually high release probability of OSN terminals in the olfactory bulb of rats $\left(P_{\max } \geq 0.8\right)[102]$.

According to the estimated parameters in Drosophila, we can calculate the number of releasing vesicles triggered by a spike by the release probability times the number of release sites. Therefore, an OSN spike can initiate the release of dozens of vesicles from the axon terminals of an OSN to its postsynaptic PN dendrites and elicit strong responses of the PN. In contrast, the excitatory synaptic contact in rat barrel cortex probably only releases one vesicle when the activation from one presynaptic fiber occurs [103]. Therefore, although an odor evoked weak responses from OSNs, of which spiking rate is about 30 spikes/s, several experiments showed that the responses of their postsynaptic PNs could spike at more than 100 spikes/s [44, 48, $53,55]$.

The available number of vesicles per synapse in a short period is limited. A high release probability usually causes short-term synaptic depression because it is more likely for these synapses to exhaust almost all releasable vesicles and to cause the 

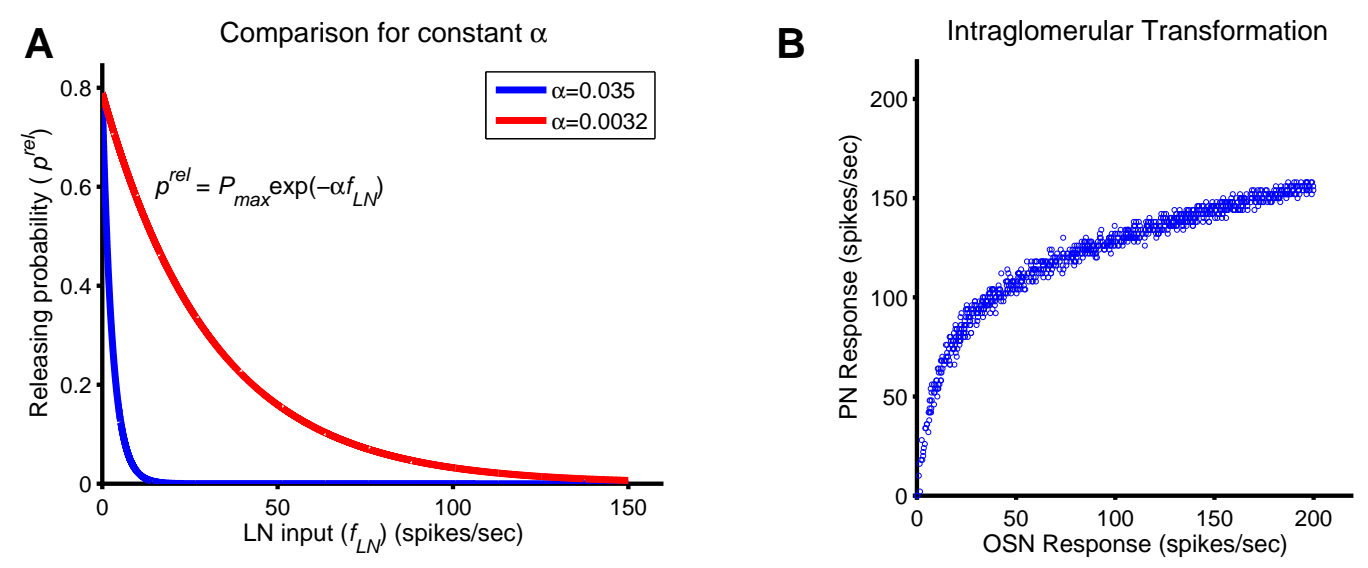

Figure 2.5: Nonlinear intraglomerular transformation and the effect of its parameter $\alpha$. A. The effect of different constants $\alpha$ on the releasing probability. In the simulation we set constant $\alpha$ to 0.0032 instead of 0.035 for exhibiting moderate and realistic inhibition. B. The input-output signal transformation in a glomerulus. Each circle indicates a simulation trial. After running one thousand trials, we clearly see that the relationship between OSN responses and PN responses in the same glomerulus is nonlinear. Weak OSN responses can be boosted at the level of PNs, but strong OSN responses only drive PNs to saturating responses.

depletion of vesicles when several subsequent presynaptic spikes arrive. This kind of synaptic depression has been found in OSN-PN synapses when the presynaptic stimuli were presented at different rates from $15 \mathrm{~Hz}$ to $50 \mathrm{~Hz}$ [44]. The same study suggested that the higher rate of stimuli leads to stronger depression, and the synapse could not amplify input responses at high spiking rates as much as those at low spiking rates. Therefore, the signal transformation of the input-output in a glomerulus becomes nonlinear, and this intraglomerular nonlinearity was recognized in several experiments $[44,47,48,53,55]$. Through such OSN-PN signal transformation, weak OSN responses can be boosted at the level of PNs, but strong OSN responses only drive PNs to saturating responses.

Now we already knew that this resulting nonlinear transformation is achieved by two neuronal dynamics: abundant vesicles released at synapses for weak OSN input and synaptic depression for strong OSN activation. Here we implement the model proposed in Sec. 2.5 to exhibit this excitatory intraglomerular transformation without including the effect of lateral inhibition. In this simulation, 30 OSNs and 3 PNs are connected within a glomerulus according to the report of Root and his colleagues studying the antennal lobe of Drosophila [12]. We list all used parameters of the simulation in table 2.1. Most values of parameters refer to the theoretical study reported by Oizumi et al. [94], but we decrease one constant $\alpha$ from 0.35 in their report to 0.032. Considering an exponentially decreasing function $p^{r e l}=P_{\max } \exp \left(-\alpha f_{L N}\right)$ simplified from Eq. 2.13, we get a very low vesicle releasing probability $p^{\text {rel }}$ at 0.03 
based on their constant $\alpha$ at 0.35 , even though the spike rate of LN input $f_{L N}$ is set to very low at only $10 \mathrm{~Hz}$. In other words, the input from LNs at any intensity can tremendously inhibit PNs, and strong inhibition will always occur at the synapses between OSNs and PNs. Because the inhibition in their experiments is too strong and unrealistic, we use $\alpha$ at 0.032 instead so that vesicle releasing probability decreases to 0.04 when the input of spiking rate from LNs increases to $100 \mathrm{~Hz}$. The effect of different constants $\alpha$ is shown in Fig. 2.5A.

According to the results in electrophysiological experiments, the resting potential of olfactory neurons is approximately $60 \mathrm{mV}$ in Drosophila $[45,104]$ and, this value is adopted in all neurons in our simulations. Other parameters including $q, N_{0}$, and $P_{\max }$ are based the estimations suggested by Kazama and Wilson [44]. The sampling rate is $5000 \mathrm{~Hz}$, and so the size of each time step in simulations yields $0.2 \mathrm{~ms}$.

We randomly select the spike rate of OSN responses from 0 to $200 \mathrm{~Hz}$ as input (sampling from a uniform distribution), measure the spike rates at the PN level, and repeat this experiments for one thousand times. In concert with experimental results $[44,48,53,55]$, the relationship between OSN responses and PN responses in our simulation is nonlinear at lower concentration input and becomes less correlated as the input increases to higher concentrations (Fig. 2.5B). Such nonlinear intraglomerular transformation controls the interaction between OSNs and PNs and plays an important role in the olfactory processing. The simulation results consistent with other experimental data confirm that the high release probability of vesicles at the synapses between OSNs and PNs, together with the synaptic depression caused by the vesicle depletion, can establish the nonlinearity in the input-output function within a glomerulus. 



\section{Concentration-invariant odor representations}

\subsection{Overview}

The odors in the external worlds are represented in the ways of diverse quality (the chemical structures of odor molecules) and great disparity in quantity (odor concentrations or intensity). However, how animals efficiently recognize a large repertoire of odors in terms of quality and quantity has not been reached a final conclusion. In this chapter, we begin with discussing what phenomena have been captured in the olfactory process for encoding quantitative information in the antennal lobe or the olfactory bulb. Then we use our proposed model to show that the recurrent networks with specific inhibition feedback is sufficient to account for the coding strategies found in many neurobiological experiments. We also implement different kinds of odor response profiles and change the weights and patterns of recurrent connection to characterize the model by comparing the results of simulations. Finally, we show that the proposed model can serve as a basis for how the olfactory system can encode odor quantity and distinguish odor quality simultaneously.

\subsection{Encoding quantitative information in OSNs}

Odor stimuli used for behavioral experiments in the olfactory system often range over six orders of magnitude in concentration. All these stimuli from low to high concentrations can drive animals to perform distinct behavioral responses including attraction and aversion to given odors, and higher concentrations often elicit responses different from low concentrations [105-107]. In contrast to the responses of animals in behavioral experiments over a broader concentration range, the dynamic of OSN responses is limited in a narrower range of concentrations [39, 105, 108]. Specifically, the OSN response has become saturated since testing concentration has increased to only approximately two or three orders from the intensity to which this neuron started to respond. [39, 105, 108]. 

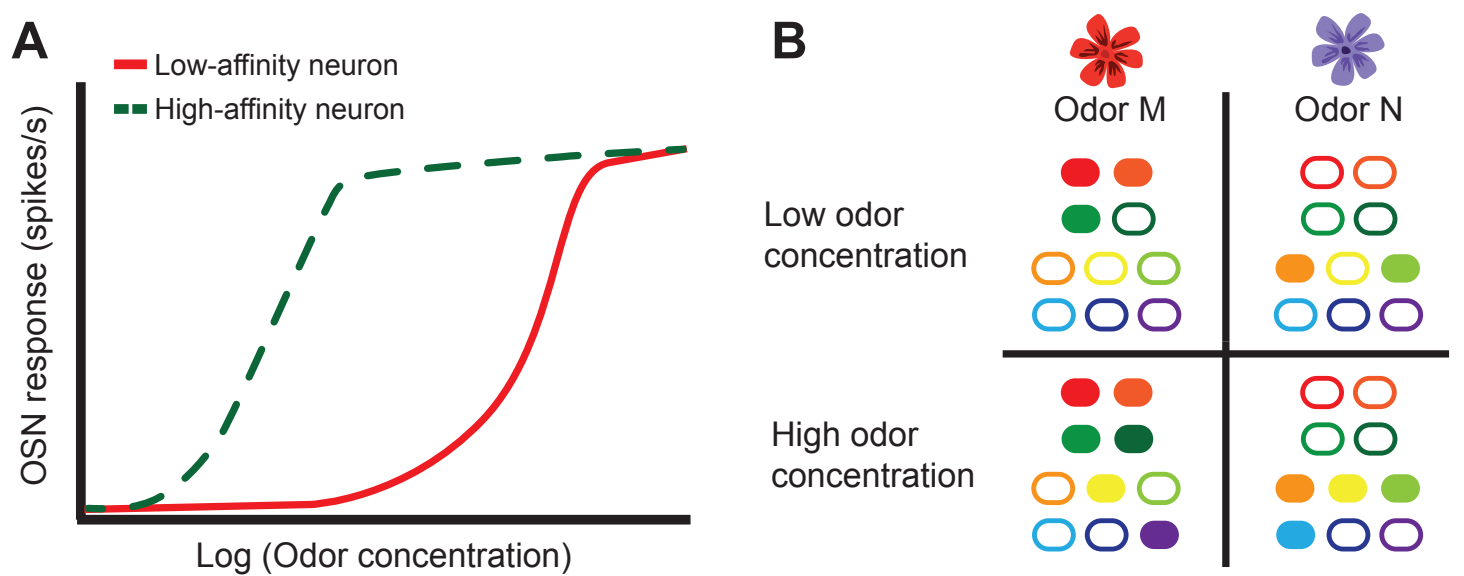

Figure 3.1: Schematic illustration of odor representations at the OSN level. A. Concentration-response profiles for two neurons preferring the same odor but having different affinities. The dynamic ranges of OSNs are narrower than the concentration range of stimuli (horizontal direction). High-affinity neurons respond differently only at lower concentrations (green dash line) whereas the responses of low-affinity neurons are distinguishable only at higher concentrations (red solid line). Combining both types of neurons can extend the dynamic range of olfactory receptive field. B. Odor-evoked patterns for different concentrations (upper and lower) and different odor qualities (left and right). Ten different OSN types are individually represented by ten different color ellipses. A filled ellipse denotes this OSN being evoked. These different patterns are several combinations of responses of different OSN types and are thus called combinatorial codes. In the same odor quality, higher concentration can activate more types of OSNs. In the same odor quantity, different odor qualities are represented by different combinations of OSN types. The difference of odor-evoked patterns between qualities is much larger than the difference between quantities.

How can the olfactory system relying on limited dynamic of an OSN (e.g. the concentration response curve depicted as the green dash line in Fig. 3.1A) represent odor stimuli in much broader range of concentration (e.g. all possible concentrations along the horizontal axis in Fig. 3.1A)? It might be that the activation of OSNs is merely used for detecting if there is in presence of a particular odor. In this case, although the olfactory system will lose part of quantitative information when the activity of OSNs reaches saturation, the perception of smelling a specific odor still functions in a straightforward way depending on whether one or several corresponding OSNs are activated.

However, a recent study indicated that the mechanisms of olfactory coding are much more complicated than the above assumption [105]. This study suggested that the antennal lobe could use two types of OSNs preferring the same odor but having 
different affinities to establish a much wider concentration receptive field (e.g. two neurons in Fig. 3.1A). In other words, although one OSN type reaches saturating responses, the differential responses from the other OSN type can still be capable of encoding different odor concentrations into different patterns. Specifically, the OSN type sensitive to weak odors is informative at lower concentrations (high-affinity neuron, green dash line in Fig. 3.1A) because different odor quantities at lower concentrations can be reflected only by the discriminating responses of the high-affinity OSNs. Similarly, the other type is much more informative at higher concentrations (low-affinity neuron, red solid line in Fig. 3.1A). If the olfactory system combines both high-affinity and low-affinity types of OSNs together to represent stimulus intensity, the dynamic range of olfactory receptive field can be largely extended. At lower concentrations, low-affinity neurons are inactive, and the odor representations will be highly dependent on the responses of high-affinity neurons whereas lowaffinity neurons are responsible for encoding the intensity at higher concentrations where high-affinity neurons are not sensitive. Therefore, the quantitative information can be jointly represented by the neuron responses of two different types. Here, the strategy of recruiting multiple OSN types to encode odors is commonly called combinatorial coding.

\subsubsection{Combinatorial coding in quantity and quality}

The combinatorial coding scheme utilizing two OSNs with different sensitivity to concentrations effectively extends the range of encoding odor concentrations in the antennal lobe. Different odor intensities are encoded into distinct patterns based on different sensitivity ranges of two OSN types, and downstream neurons should be capable of decoding intensity information from those distinguishable patterns. Although the combinatorial coding indeed solves the problem caused by narrow sensitivity ranges of OSNs, it could also bring the adverse effect, increasing the complexity in the olfactory coding space.

It has been found that in the olfactory systems across species, different odors elicit different activation patterns in OSN assemblies [10,29,75, 108-113], and the odor quality is thus preserved in the distinction between neural representations. Therefore, odor qualitative information is encoded in the same way (combinatorial coding) as odor quantitative information at the OSN level. The qualitative and quantitative information is then mixed up in the input olfactory representations generated from peripheral OSNs. As a consequence, higher brain areas have to solve another crucial problem that judging the change of representations is initiated by the change of odor quality or quantity. Could the new presence of a distinct representation mean an arrival of a new odor or exactly the same odor but its concentration being altered?

The question can be replied by many studies suggesting that the difference of combinatorial codes between qualities is much larger than the difference between quan- 
tities (Fig. 3.1B) [10,12,39,75, 107]. An increasing concentration can often recruit two or three more OSN types whereas different odors are encoded in a variety of combinations out of large numbers of distinct OSN types, for example, about 50 for Drosophila.

If the activity of one OSN type represents one dimension in olfactory space, the high-dimensional olfactory coding space should be large enough to accommodate a wide variety of olfactory quality and quantity. The dimension of olfactory space in mammals is much larger than the dimension in insects. For example, in human beings the dimension of OSN representations is several hundred [114,115] and up to one thousand for mice and rats $[8,116]$. Such high dimensionality should be capable of satisfying the demand for allocating all olfactory representations appearing in the natural environment.

To sum up, OSN patterns are concentration dependent and are represented in combinatorial codes. Odors at a low concentration only elicit responses of a few types of OSNs whereas most OSN types are inactive. The increasing spike rates of OSNs with odor concentration progressively morph neuronal activity patterns. As concentration increases, the responses of some OSN types reach saturation, and some new OSN types may be recruited in the olfactory coding.

The response sensitivity to an odor depends on the ligand-receptor affinity of an odor receptor, and different types of odor receptors contribute to different response profiles of OSN types. An odor at low concentration can elicit the responses of OSNs carrying the receptors with high ligand-receptor affinity while increasing odor concentration can progressively recruit OSN ensembles with low ligand-receptor affinity. The dynamic range of high-affinity OSNs spans mostly over lower concentrations, and low-affinity OSNs are more active at higher concentrations (Fig. 3.1A). Two OSNs with different affinity working together can integrate their concentration-response profiles to extend the dynamic range of encoding olfactory input.

In this chapter, we focus on how neural circuits process and encode quantitative information, and how the antennal lobe uses the combinatorial codes to achieve specific schemes in olfactory processing. We will continue to cover the topics regarding odor quality coding and discuss how the antennal lobe can distinguish odor quality based on combinatorial codes in the following chapters.

\subsection{Concentration-invariant coding}

The odor signal travels through the medium of air or water to the peripheral olfactory system. Because the unstable properties of fluid, the signal transmission medium is indeed unsteady with several kinds of turbulence of which structures 


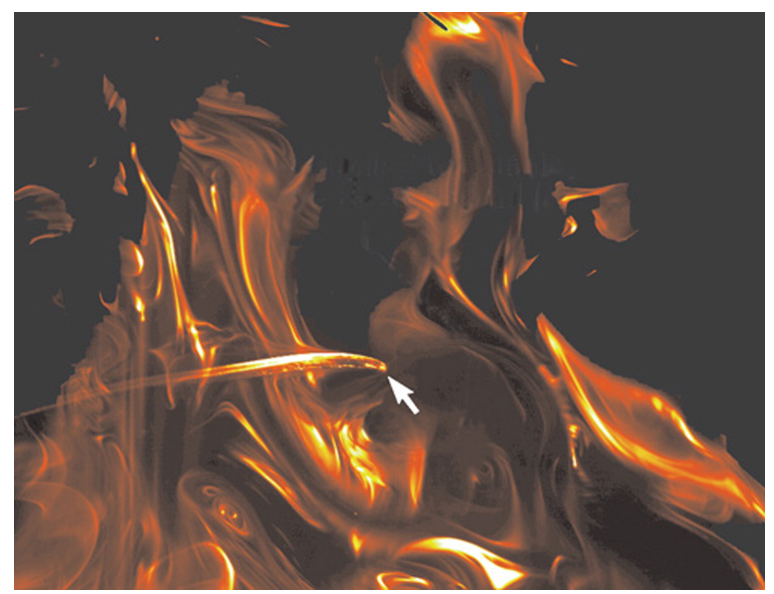

Figure 3.2: Odor concentration is discontinuously distributed in the air or water space because of turbulent flow and stochastic odor molecule diffusion. Brighter intensity indicates higher odor concentration. The arrow denotes an antennule of a lobster exposed to this odor. ${ }^{1}$ (Photographing image taken from [85])

vary in sizes. Odor signals are also transported and spread in the way of molecular diffusion $[117,118]$. These varying physical factors always change how and how many odor molecules are transported to the peripheral area of olfaction. The OSNs in the peripheral area will therefore sense different scales of concentration fluctuation in hundreds of milliseconds. Similarly, the odor concentration is discontinuously distributed in the air or water medium (Fig. 3.2), and when animals move around this environment, the concentration their OSNs sense would always also vary. This contrasts with the visual modality where photoreceptors are often stimulated by nearly constant light intensity of visual objects in normal environment. For example, the light intensity of words you are currently reading almost keeps constant either on the paper or on the screen. In general, most change of received odor signal strength results from the effect of turbulence in transport medium and the stochastic odor molecule diffusion instead of the concentration variation of odor sources.

We earlier talked about that combining two types of OSNs with different affinity could extend the dynamic range of the system to respond to broader range of input concentration. This means that OSN ensembles can mostly encode varying input concentration and relay this concentration dependent information to higher brain areas. However, if the olfactory system keeps tracking any tiny change of odor concentration, neural systems would spend vast neural resource representing and processing this quantitative information, and it could hugely increase the amount of unnecessary and redundant sensory information in the brain. Even worse, most varying concentration information is created by random diffusion of odor molecules or turbulent flow and is unrelated to the true quantity of odor sources. In contrast

${ }^{1}$ Reprinted from Neuron, 48(3), B. W. Ache and J. M. Young, Olfaction: diverse species, conserved principles, p418, Copyright (2005), with permission from Elsevier. 
to other modalities, the olfactory modality needs to solve this specific and onerous issue when processing quantitative information.

\subsubsection{Representation invariant to concentration fluctuation}

How do the neural circuits filter out the input concentration fluctuation during the olfactory processing? A solution found across different species in nature is that odor stimuli in similar concentrations are generalized as the same representation in olfactory computation. Many studies suggested that the representations can be classified in the same clusters at the output of the antennal lobe or the olfactory bulb even when the concentration of input odors fluctuates [72,75, 76, 106, 108, 119-124]. In one of these studies, Stopfer et al. used five concentrations over a 10,000-fold range of three odors in experiments and recorded the responses of PNs in locusts. Their data showed that the response patterns of PNs changed little within 10 or 100-fold concentration range of all testing odors [76]. How wide a range of input odor concentrations (10 or 100-fold concentration) can be considered as similar odor stimuli depending on which odor quality is presented.

Another report in investigating honeybees also indicated that the antennal lobe encoded odors into separated clusters at the PN level when the animals were stimulated by odor concentrations diluted from $10^{-7}$ to $10^{0}$ [75]. Similar results of invariant output responses have been also found in vertebrates. In the olfactory bulb of zebrafish, the responses of output neurons (MCs) to 10 or 100-fold change in concentration were similar, and the response patterns were generalized into two clusters visualized by a linear dimensionality reduction method, principal component analysis (PCA) [125] as input odor concentration was increased from $10^{-7}$ molar concentration (moles per liter, $\mathbf{M})$ to $10^{-3} \mathbf{M}[119]$.

More evidence comes from the analysis of input odor-evoked patterns. In rats, several reports suggested that although the raising of odor concentration could recruit the activation of new OSNs and intensify the responses across activated OSNs tuned to that odor, performing normalization on odor-evoked OSN patterns alleviated the effect of increasing concentration, and these normalized patterns remained almost constant across different concentrations $[72,120,121]$. The normalization operation is calculated by the mean of odor-evoked signals (e.g. the mean of entire imaging signals in their studies) subtracted from the value of each basic unit of signals (e.g. the intensity of each pixel in an image), then divided by the variance of all signals:

$$
x_{i}=\frac{s_{i}-\mu_{s}}{\sigma_{s}}
$$

where $s_{i}$ is the signal for $i$ th basic unit (e.g. for the $i$ th pixel); $\mu_{s}$ and $\sigma_{s}$ are the mean and variance of signals respectively. 
Consistent results have been shown in the olfactory bulb of turtles when the same operation was applied to the images of input activity patterns. The normalized odorevoked patterns also changed little over at least a 200-fold concentration range [108]. The mechanism against concentration fluctuation may be therefore achieved by the normalization operation. Such operation requiring the subtraction of the mean from input strength implies that feedback inhibition should play a main role in the olfactory processing of the recurrent systems.

Results of behavioral experiments also support the assumption of such concentrationinvariant strategy. A study indicated that the larva of Drosophila exhibited consistent attraction to an odor from $60 \mu \mathrm{M}$ to $30 \mathrm{mM}$, and the concentration-invariant behavior preserved over a concentration range up to $2.7 \mathrm{log}$ units [106]. Another study reported that honeybees behaved similarly between the conditioning odor and the same odor in 100-fold concentration [123]. These honeybees were conditioned by odors at an intermediate concentration, and after being conditioned, they learned and preferred to extend their proboscises in the conditioning odors. Surprisingly, the honeybees showed repulsive behaviors to the conditioned odors at a lower concentration as well as to novel odors at an intermediate concentration. The results of their study suggests that the concentration-invariant recognition may be in a limited concentration range.

Comparable results have also been observed in rats. The presence of a novel odor raised significantly differential responses of rats than the presence of a habituating odor did at a different concentration [121]. All these behavioral results suggest that in olfactory systems, the quantitative information could be generalized for the same odor quality, but it is likely to encode each odor quality as distinct representations. Similarly, as rats could generalize the same odor at different concentrations, they identified odor mixtures according to the ratios of components and had consistent responses to over at least ten-fold range of concentration [122]. From neurophysiological data to behavioral experiment results, it becomes clear that concentrationinvariant coding is well established in the neural computation of the antennal lobe or the olfactory bulb incorporating feedback inhibition so that the olfactory system can suppress any concentration fluctuation.

\subsubsection{Concentration invariance within confined ranges}

So far, many pieces of evidence corroborate that the olfactory coding can transform olfactory information into stable representations independent of small concentration variations. However, the odor intensity still reflects significant information from external environment and may convey vital messages such as the distances between animals and their food sources or even their predators. Indeed, the odor intensity is Gaussian-like distributed from the center of the odor source and decreases symmetrically because of the way of odor molecule diffusion [106]. The olfactory 


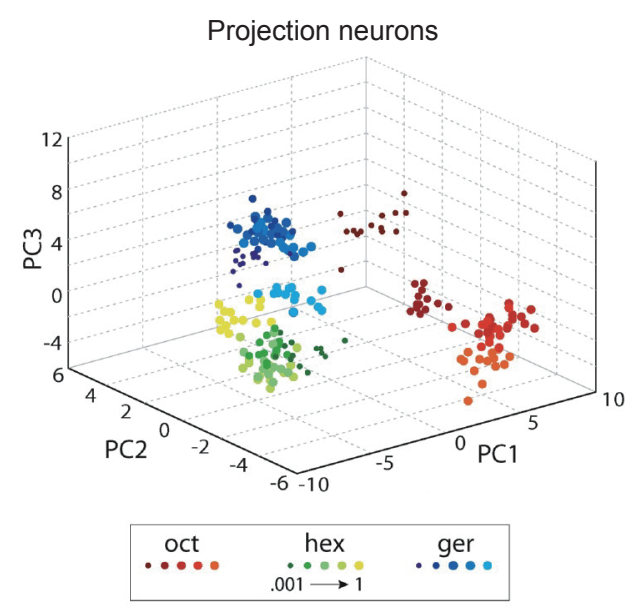

Figure 3.3: Concentration-invariant representations in discrete clusters. The neural circuits categorize odors at five different concentrations into two discrete clusters at output layer. Odors at lower concentrations are classified to one cluster, while odors at higher concentrations are classified to the other. Within each cluster, the odor representations are invariant to concentration change. ${ }^{2}$ (Figure modified from [76])

modality should be capable of decoding sensory information to know the relative distances from odor signals based on how much odor intensity is received. Moreover, odor-evoked patterns in OSN ensembles already bear and encode quantitative information, and it is unlikely that all of this useful information is thoroughly overlooked in the olfactory processing. In contrast to the visual modality, measuring how far nearby objects are from individual animals is basically based on the geometric size of these objects instead of their light intensity. If animals want to exploit any clues as to how far odor sources are from themselves, it is reasonable that they smell out the relative distances from odor signals in environment based on the strength of neuronal activation in odor-evoked patterns.

In fact, several studies in section 3.3.1 already showed that the antennal lobe or the olfactory bulb generalizes the same odor at a series of concentrations into two clusters $[75,76,106,119]$. One cluster represents lower concentration odors, and the other accounts for odors at higher concentrations (Fig. 3.3) [76]. Some reports did not mention similar phenomena in their data probably because the concentration range they used for odor stimulation is narrower than the range one cluster generalizes. In addition, the idea, concentration invariance within a few clusters, is supported by more evidence from the experiments in Drosophila and human beings. One study investigated behavioral responses to different odor intensities in Drosophila, showing that weak and strong concentrations of the same odor can elicit attraction and aversion behavior respectively [107]. In human psychophysical

2 Reprinted from Neuron, 39(6), M. Stopfer, V. Jayaraman, and G. Laurent, Intensity versus identity coding in an olfactory system, p995, Copyright (2003), with permission from Elsevier. 
experiments, subjects reported that they smelled dissimilar odors while the same odor was presented at different concentrations [126,127]. For example, odors such as civet and ambergris smell unpleasant and even fecal at high concentration, but they become pleasant and are used to manufacture perfume when these odors are diluted. Therefore, these data suggest that different quantitative information may be encoded as different odor quality in the olfactory processing. As different odors encoded into different representations, odors at higher concentrations and at lower concentrations are likely generalized into respective representations, and higher brain areas can still distinguish different intensities of the same odor.

Although the olfactory system implements the mechanism of concentration-invariant coding, this mechanism is indeed partial and within a confined range of concentration. The same odors over a large concentration range are generalized into a few clusters, composed of one representative for a strong odor and the other representative for a weak odor. This means that there are only a few distinct odor representations for one odor quality in olfactory output neurons; therefore, the generalization mechanism may allow classification of odor intensity more efficient in higher brain areas. Quantitative information represented at the OSN level can be still relayed to higher processing areas in discrete and binary representations, and the brain can be aware of odor quantity change from the olfactory peripheral.

In the next section, we will demonstrate how the odor representations can be invariant to the concentration of odor stimuli in the olfactory modality based on our theoretical model. We will also show that how the neural circuits generalize similar odor intensity and encode weak and strong odors into discrete representations.

\subsection{Results in theoretical investigations}

The antennal lobe in insects and olfactory bulb in vertebrates share conserved architecture and implement similar signal transformation between intra- or interglomeruli $[46,84]$. To show how sensory information is evolved and processed in the olfactory system, here we demonstrate the olfactory computation in the antennal lobe, and PNs are used as the output neurons instead of MCs.

\subsubsection{Model setup}

In all simulations, the antennal lobe network consists of 30 OSNs and 3 PNs per glomerulus as the setting we used in section. 2.6 Several LNs are implemented to build the recurrent connections, and totally 9 glomeruli compose the recurrent networks of the antennal lobe. The parameters of equations describing LN membrane 
Table 3.1: Parameters of LNs in the antennal lobe network.

\begin{tabular}{lll} 
LN & & \\
Name & Value & Description \\
\hline \hline$\tau_{m, L N}$ & $5 \mathrm{~ms}$ & membrane time constant \\
$V_{0, L N}$ & $-60 \mathrm{mV}$ & resting potential \\
$V_{r e s e t, L N}$ & $-45 \mathrm{mV}$ & resetting potential \\
$V_{t h, L N}$ & $-80 \mathrm{mV}$ & threshold potential \\
$t_{r, L N}$ & $1 \mathrm{~ms}$ & absolute refractory period \\
$R_{m, L N} I^{O S N}$ & $8 \mathrm{mV}$ & external input when a spike arrives \\
\hline
\end{tabular}

potential are listed in Table 3.1. The values of parameters are the same as the parameters we use in the PN equations. During simulation, each neuron generates its spike sequences based on equations in section 2.5. Because we focus on the olfactory processing in terms of spatial information of evolving neural representations and neuronal interactions within and across several glomeruli in this thesis, the responses of neurons are presented by spike rates. However, it does not mean the temporal information is not important in the olfactory processing. Instead, our approach is a spike-based model and can be flexibly extended to demonstrate more details in the temporal domain such as oscillatory synchronization $[128,129]$.

The neuronal variability of OSN spiking comes from the Poisson process. To minimize the effect of OSN spiking variability from each single trial, all results of neuron responses we show in this study are presented by the averages over thirty trials. We confirmed that the averaged data are consistent for each simulation. Moreover, the central limit theorem states if the sampling size is large enough, typically the size of thirty samples is sufficient, the sampling distribution will be approximately a normal distribution, and we can estimate the mean of the sampling distribution by the mean of sampling data. Thus, we choose the sampling size as thirty because of the balance between the statistical significance and the consumption of computational resources.

\subsubsection{Experiment: single odor quality}

In all simulations, we implement nine glomeruli and several inhibitory LNs including one or two LNs laterally connecting to part of glomeruli and one LN connecting to all of glomeruli in the recurrent networks; therefore, the connectivity patterns of LNs are heterogeneous. In the first simulation experiment, the response profiles for each OSN type in corresponding glomerulus and their maximal responses to odor $\mathrm{A}$ are listed in Table 3.2 and illustrated in Fig. 3.4A. The OSN activity is 
Table 3.2: OSN responses to odor A in each glomerulus and LN lateral connections to glomeruli.

\begin{tabular}{cccccccccc}
\hline Glomerulus No. & 1 & 2 & 3 & 4 & 5 & 6 & 7 & 8 & 9 \\
\hline \hline $\begin{array}{c}\text { OSN responses to } \\
\text { odor A }\end{array}$ & + & ++ & + & $\bullet$ & +++ & + & + & $\bullet$ & ++ \\
\hline $\begin{array}{c}\text { LN1 connection }^{b} \\
\quad\left(W_{L N 1}: 2.4\right)\end{array}$ & & $\mathrm{x}$ & & $\mathrm{x}$ & & & & & $\mathrm{x}$ \\
$\begin{array}{c}\text { LN2 connection } \\
\left(W_{L N 2}: 0.2\right)\end{array}$ & $\mathrm{x}$ & $\mathrm{x}$ & $\mathrm{x}$ & $\mathrm{x}$ & $\mathrm{x}$ & $\mathrm{x}$ & $\mathrm{x}$ & $\mathrm{x}$ & $\mathrm{x}$ \\
\hline
\end{tabular}

a The symbols represent the maximal response to odor A at the highest concentration: "•" 5 spikes/sec; "+" 50 spikes/sec; "++" 100 spikes/sec; "+++" 150 spikes/sec.

${ }^{b}$ The "x" means the connection between the LN and this glomerulus. The weight of LN1 $\left(W_{L N 1}\right)$ connecting to part of glomeruli is 2.4 , and the weight of LN2 ( $\left.W_{L N 2}\right)$ connecting to all glomeruli is 0.2 .

assumed to increase linearly with concentration in logarithmic scale $[65,75,130]$. In each glomerulus (each column of Table 3.2), there are 30 OSNs of the same type converging their axons onto their corresponding glomerulus, and 3 PNs receive the odor signals from their 30 cognate OSNs in the same glomerulus. LN1 connects to glomeruli No. 2, 4, and 9 meaning that LN1 only receives the neuronal activity from the OSNs in glomeruli No. 2, 4, and 9 and specifically inhibits the PNs in same glomeruli if this LN is activated.

The responses of OSNs and PNs to odor $\mathrm{A}$ at different concentrations are shown in respective PCA spaces (Fig. 3.4B and 3.4C). The neuron responses are measured by calculating the spike rates in the period of odor stimulation. OSN representations at different concentrations are almost equally distributed along a line in the space whereas PN representations are generalized into two clusters. The PN responses to 0.01 and 0.1 concentrations form a cluster, and the PN responses to higher concentrations are classified in the other cluster.

Another measurement we use here is Pearson correlation coefficient [131], which measures the linear dependence of pairs between odor responses to different concentrations. This correlation coefficient index is remarkably irrelevant to response magnitude. For natural signals, the correlation is between 0 and 1 ; 0 for no correlation between pairs, and 1 for pairs being completely linearly dependent. The Pearson correlation coefficient $\rho_{x, y}$ between odor response patterns at concentrations $x$ and $y$ is defined as:

$$
\rho_{x y}=\frac{\operatorname{Cov}\left(R_{x}, R_{y}\right)}{\sigma_{R_{x}} \sigma_{R_{y}}}
$$


A

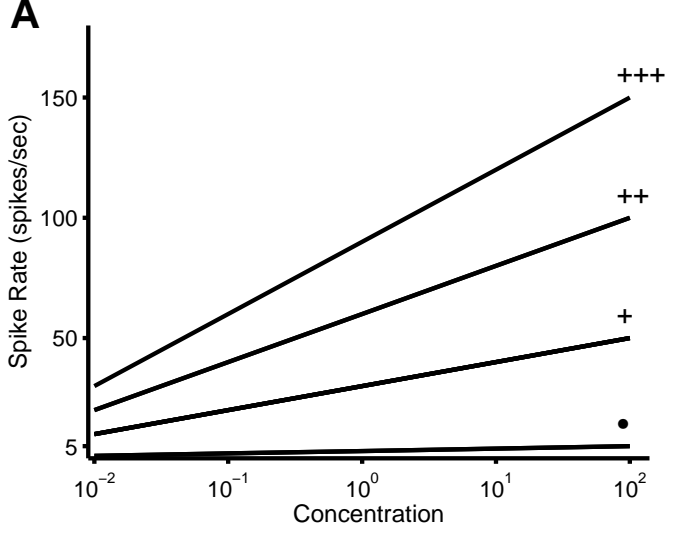

C Projection neurons

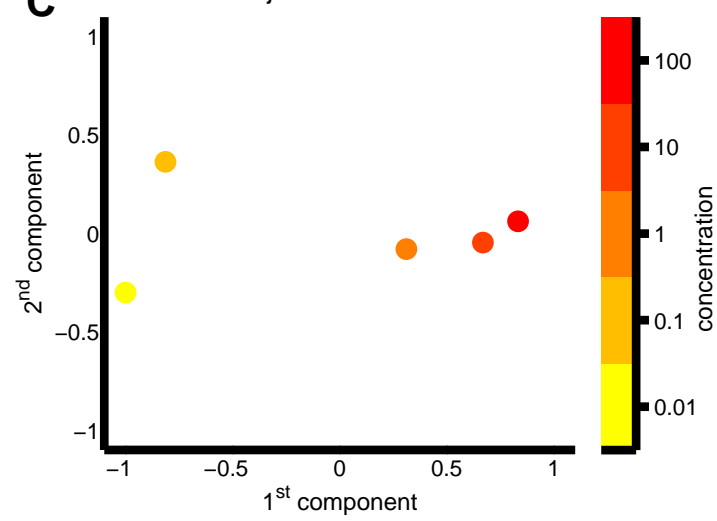

E

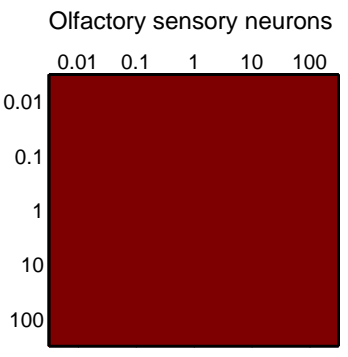

Pearson correlation coefficient

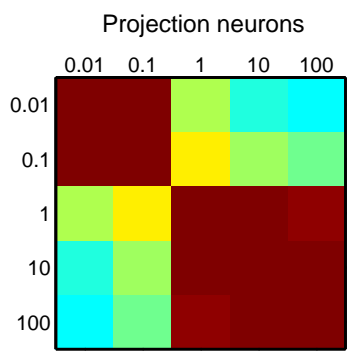

$\begin{array}{lll}0.25 & 0.5 & 0.75\end{array}$
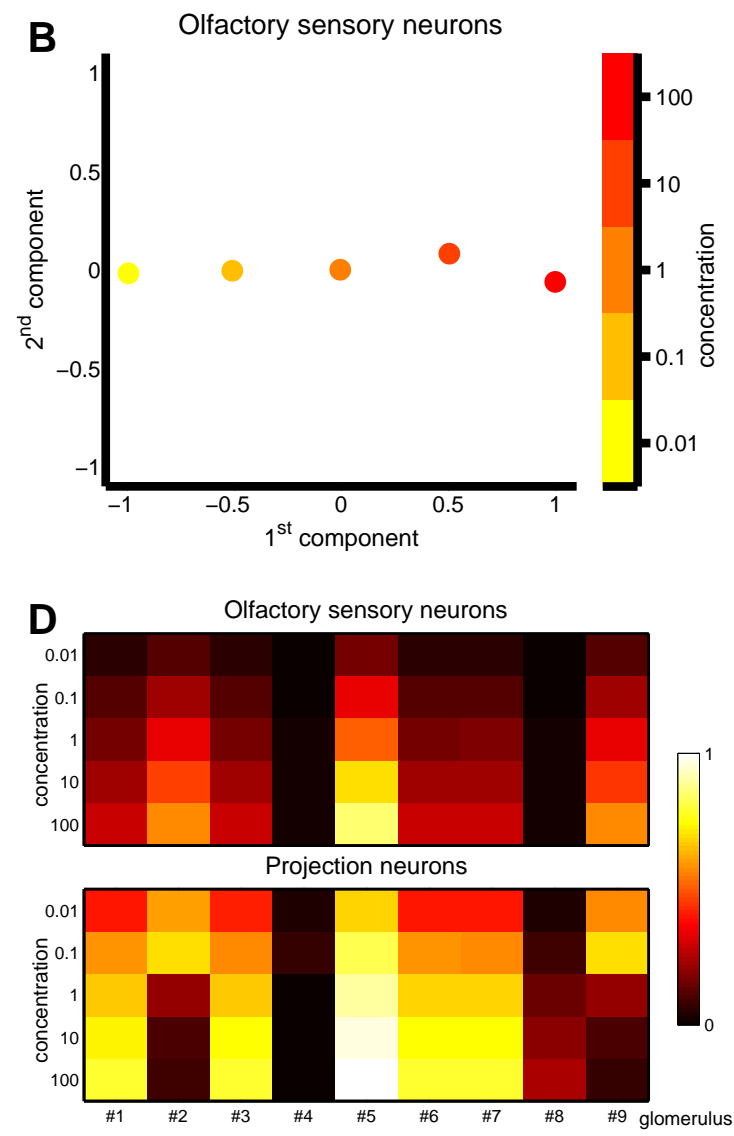

$\mathbf{F}$

Local neurons

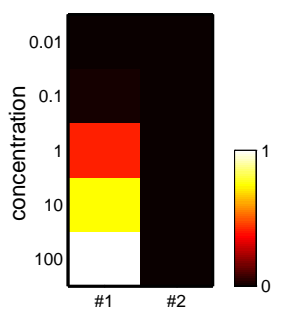


where $C o v$ is covariance, $\sigma$ is standard deviation, and $R_{x}$ and $R_{y}$ are the response patterns at concentration $x$ and $y$ respectively. $R_{x}$ and $R_{y}$ are vectors, consisting of the neuronal activity along glomeruli, and the neuronal activity in each glomerulus is represented by the spike rates averaged over all neurons in individual glomeruli. The correlation we refer in the thesis is Pearson correlation coefficient.

The correlation matrices in Fig. 3.4E depict the pairwise pattern similarity between concentrations. The Pearson correlation coefficients between different concentrations at the OSN level are close to one meaning that these OSN representations are highly correlated. It is not surprising because all the patterns are linearly related based on the assumed response profiles of OSNs (Fig. 3.4A). Compared to the highly correlated patterns at the level of input neurons, the antennal lobe decorrelates these pattern so that PN activity patterns representing higher concentrations are separated from those representing lower concentrations. Although the decorrelation indeed occurs, the antennal lobe still renders the concentration-invariant

Figure 3.4 (previous page): Olfactory information is separated into two clusters at output PNs whereas the activity of input OSNs is continuous and dependent on concentration. Lateral connection weights: $W_{L N 1}=2.4 ; W_{L N 2}=0.2$. A. Response profiles for each OSN type. The OSN activity increases linearly with concentration in logarithmic scale. B. OSN responses projected onto the space defined in the first two principal components. C. PN responses projected onto the space defined in the first two principal components explaining $98.8 \%$ variance of original data. The $\mathrm{PN}$ responses are separated whereas the OSN responses in $(\mathbf{B})$ are almost equally distributed. Note that only when the generalization of data points appears in the first two principal component space, we need to specify the variance of original data represented in the first two principal components. This is because low variance could indicate that the data points clustered in the space spanned by the first two principal components disperse in other dimensions. Generally speaking, a PCA space should explain at least $80 \%$ variance of original data to reflect the data structure. D. Response matrices showing the responses (spike rates) of OSNs and PNs in each glomerulus (column) to each concentrations (row). The represented responses are the averaged responses of all neurons in the same glomeruli and normalized to the highest response in the response matrices. Note that the responses of the PNs in glomeruli No. 2 and No. 9 have abrupt transition over concentration. E. Correlation matrices representing the pairwise similarity between OSN responses and between PN responses to different concentrations. The two red blocks indicate that concentrations are separated into two clusters. The correlations between concentrations within the same clusters are close to 1 , and the correlations between concentrations from two different clusters are much lower. F. A matrix showing the responses of LNs (column) to each concentration (row). The responses are normalized to the highest response in the response matrix. 
coding within confined ranges.

The abrupt transition between patterns at concentrations 0.1 and 1 attributes to the activation of the LN (LN1) connecting part of glomeruli. When the LN is activated due to concentration increase (Fig. $3.4 \mathrm{~F}$ ), the strong lateral inhibition starts to act on PNs in glomeruli No.2, 4, and 9. With the effect of inhibition, the activity of these PNs becomes diminished, and these neurons no longer send information out of the antennal lobe as much as they receive after the concentration of odor A exceeds 1 (Fig. 3.4D). In contrast, PNs in glomeruli No. 2 and No. 9 have intermediate responses at concentrations 0.01 and 0.1 . Therefore, such difference of PN responses substantially transforms the output patterns when glomerulus-specific lateral inhibition begins involving in the olfactory processing. Surprisingly, the lateral inhibition also generates invariant representations at the PN level because the PN activity keeps stable and silent across higher concentrations in the effect of inhibition.

Moreover, the intraglomerular nonlinear transformation also plays a significant role in concentration-invariant coding. Other PNs intact from the lateral inhibition such as those in glomeruli No. 1, 3, 5, 6, and 7 increase their responses not so much as their cognate OSNs at concentrations from 1 to 100 . This is because the PN responses already approach saturation or become saturating at higher concentrations whether their cognate OSNs have intermediate responses in glomeruli No. 1, 3, 6, and 7 or stronger responses in glomerulus No.5. The nonlinear transformation is already shown in Fig 2.5B. As a consequence, invariant representations across higher concentrations also stem from nearly stable activity of PNs in glomeruli No. 1, 3, 5, 6 , and 7 .

Interestingly, the intraglomerular signal transformation also helps the antennal lobe generalize odors into two clusters. The low spike rates of OSNs in glomeruli No. 2 and No. 9 at concentrations 0.01 and 0.1 are amplified at nonlinear transformation glomeruli, and the activity of their postsynaptic PNs is highly boosted. When the concentration is increased to 1 , the activation of LN1 suppresses the responses of PNs in glomeruli No. 2 and No. 9. Two opposite mechanisms of boosting activation and suppression on the same PNs make response patterns more distinct between lower concentrations and higher concentrations. Through the nonlinearity in the intraglomerular transformation coupling together with lateral inhibition, the antennal lobe can separate two clusters even further and generate abrupt transition between representations of linearly related input signals.

Results of the first experiment are consistent with the idea of concentration-invariant coding. We have shown that the interglomerular inhibition and nonlinear intraglomerular transformation indeed achieve such strategy in the antennal lobe. The activation of an LN is associated with the OSNs in the glomeruli where this LN laterally innervates. Among all these OSNs, when an odor is presented, this LN has more interaction with the OSN types preferring this odor. For example, given the stimulation of odor A, the responses of LN1 highly depend on the OSNs in 
glomeruli No. 2 and No. 9 than those in No.4. Similarly, the responses of PNs are stronger in glomeruli No. 2 and No. 9 than in No. 4. On this basis, if we increase the concentration of odor stimulus from the lowest concentration, we can see that the activity of PNs in glomeruli No. 2 and No. 9 quickly rises. After the concentration is across a threshold of activating LN1, LN1 begins providing feedback inhibition. So the responses of the same PNs diminish and become silent. The dramatic change of PN responses in glomeruli No. 2 and No. 9 between lower and higher concentrations results in abrupt transition between pattern morphing.

Which neurons will be involved in the neural computation is the collective consequence of the interaction between the combinatorial odor-evoked OSN activity and the lateral connectivity of LNs. Although the glomerulus No. 4 is laterally connected by LN1, the low sensitivity of OSNs in glomerulus No. 4 to odor A excludes these OSN from joining the olfactory processing of odor A. The interaction between the LN and specific OSNs, for example, OSNs in glomeruli No. 2 and No. 9 in this simulation, determines how the antennal lobe separates different odor concentrations into clusters. Notably, such specific interaction will be further applied in the later experiments.

PNs in other glomeruli are still important in olfactory coding. Most of them are also responsible for generating representations independent of odor quantity at higher concentrations because they already reach saturation.

\subsubsection{Weights of lateral connections}

To exam the effect of lateral connection strength in the antennal lobe network, we use the same OSN response profiles and lateral connections of LNs as the previous setting, and only change the weights of lateral connections in the following experiments. First, we only raise the weights of LN1 connection from 2.4 to 3.2. The increase of connection strength makes this LN more easily activated. The olfactory network with new connection weights still generalizes the odor in different concentrations into two clusters (Fig. 3.5A and 3.5C), but one olfactory representation is shifted from one cluster to another. Due to the increased connection weights of LN1, the response range of LN1 becomes broader and is across four orders of concentrations instead of three orders in the previous experiment (Fig. 3.5D). Therefore, the response patterns of PNs at concentrations from 0.1 to 100 are generalized because PN populations are inhibited by LN1 in this concentration range.

In comparing Fig. 3.5B with Fig. 3.4D, the responses of PNs in glomeruli No. 2, 4, and 9 at concentration 0.1 in this experiment are lower than the responses in the earlier experiment and are suppressed instead of being boosted. The point representing concentration 0.1 in PCA moves towards the cluster generalizing strong odors because the recurrent network at concentrations from 0.1 to 100 is all dominated by 

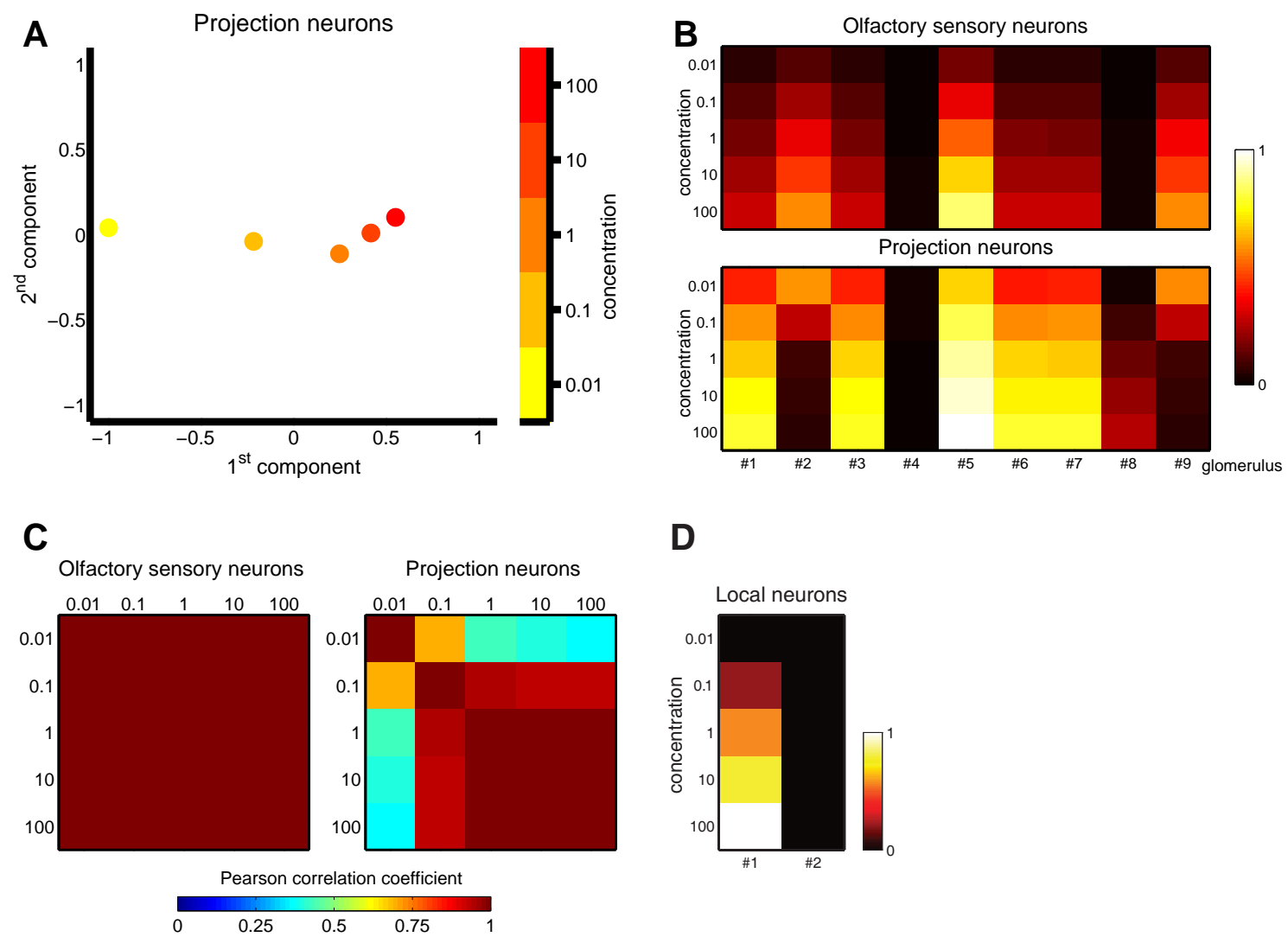

\section{D}

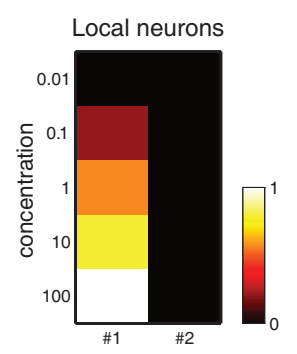

Figure 3.5: One olfactory representation is shifted from one cluster to another when the weights of the partially-connecting LN1 are increased from 2.4 in Fig. 3.4. Lateral connection weights: $W_{L N 1}=3.2 ; W_{L N 2}=0.2$. A. PN responses in the PCA space explaining $99.9 \%$ variance of original data. The representation of concentration 0.1 in PCA moves towards the cluster generalizing strong odors B. Matrices showing the responses of OSNs and PNs, respectively. C. Correlation matrices representing the pairwise similarity between OSN responses and between PN responses to different concentrations. The PN outputs representing concentrations from 0.1 to 100 are highly correlated. D. A matrix showing the responses of LNs. Because the increased weights cause LN1 activated starting from concentration 0.1, the output cluster representing strong odors now include the odor at concentrations 0.1. Plot representations are the same as Fig. 3.4.

the lateral inhibition. Therefore, this type of $\mathrm{LN}$ is responsible for quantitatively categorizing odors, and whether an odor concentration is categorized into the group standing for higher concentrations depends on if the LN is activated at this concentration. On the other hand, the strength of LN lateral connections determines how much odor concentration LNs need to be activated, and the connection weights indirectly modulate the separation line between two clusters. 

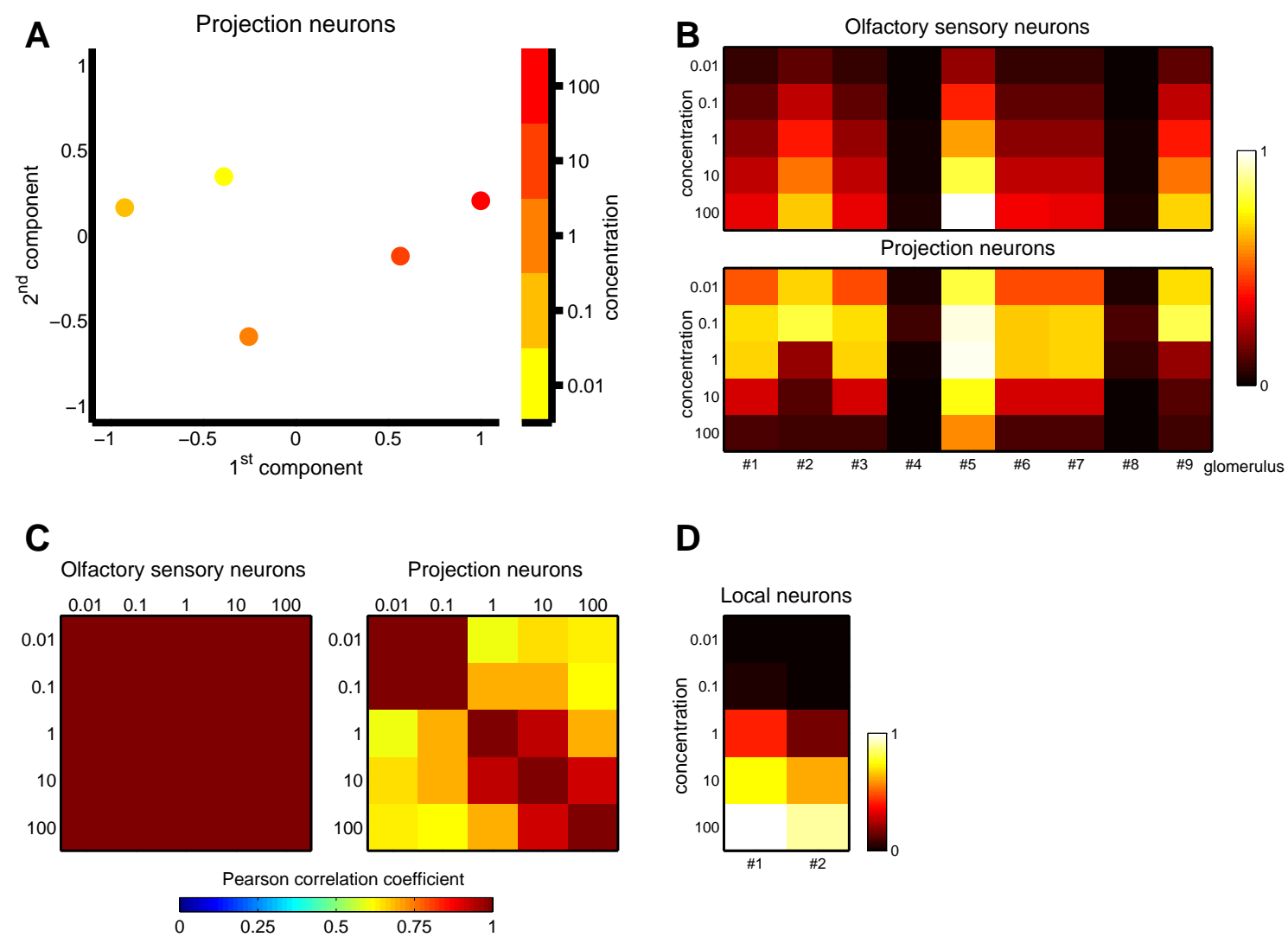

D

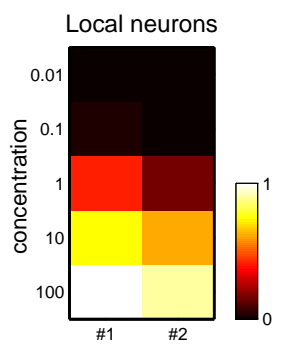

Figure 3.6: The concentration-invariant representations do not appear at the output level when the weights of the fully-connecting LN2 are increased from 0.2 in Fig. 3.4. Lateral connection weights: $W_{L N 1}=2.4 ; W_{L N 2}=0.8$. A. PN responses in the PCA space. B. Response matrices of OSNs and PNs. C. Correlation matrices representing the pairwise similarity between OSN responses and between PN responses to different concentrations. D. Response matrix of LNs. The activation of LN2 provides feedback global inhibition. The concentration-invariant representations vanish at the output level when global inhibition is recruited by increasing weights of fully-connecting local neurons. Plot representations are the same as Fig. 3.4.

Now we consider the antennal lobe as a dynamic system. Small concentration change produces no qualitative change in representations at the output level until a critical concentration is reached. At this critical point, the output representations change qualitatively, and we can say that the antennal lobe has gone through a bifurcation in terms of a dynamic system. Furthermore, according to the experimental results, the bifurcation point is a function of the connection strength of partially-connecting LNs.

Next, we retain all parameters the same as the first experiment, but only increase 
the weights of fully-connecting LN2 connection from 0.2 to 0.8 to see the effect of increasing global inhibition. Because the number of glomeruli connected by LN2 is three times as many as those connected by LN1, the increase of input strength to LN2 is more than that to LN1 given the same increase of input OSN spikes. More specifically, if one more spike is emitted by one OSN per glomerulus, LN2 then receives nine more spikes times quadruple weights, and LN1 only receives three more spikes times unchanged weights. Therefore, the global inhibition should be more actively involved in the olfactory processing than previous experiments.

The concentration-invariant representations do not appear at the output level when we enlist strong global inhibition by increasing the weights of fully-connecting LN2 (Fig. 3.6A and 3.6C). The global inhibition is the negative feedback based on the summation of all glomerular inputs, and the input from OSN responses increases with the rise of odor concentration. Therefore, the inhibitory strength should be also increased as odor concentration rises. The global inhibition results from the activation activity of LN2, and the increasing responses of LN2 in Fig. 3.6D indicate that the inhibition strength is indeed increased by odor concentration).

The negative effect of the global inhibition could be to suppress the PNs originally boosted to saturation and kept in a stable status at higher concentrations. If the strength of global inhibition changes little and is weak enough that PN responses remain saturating, global inhibition would not interfere with the mechanisms for transforming concentration dependent information into concentration-invariant representation. However, the strength of the global inhibition rises as concentration is increased, and makes PN responses different at higher concentrations (Fig 3.6B).

Concentration invariance achieved by partially-connecting LNs is to suppress part of PN responses completely, and these silent and stabilized PN neurons can result in invariant representations. We then ask: is it possible that global inhibition performs the same mechanisms as local inhibition by completely inhibiting PNs? Because this kind of inhibition is global and aims at all glomeruli, the consequence for implementing inhibition on all PNs will be no output representations at higher concentrations. The idea of representing stronger stimuli with a silent response pattern is not feasible because it means that no odor stimulus will be encoded the same way as stronger stimuli at the output level of the antennal lobe. Moreover, it would be impossible for higher brain areas to distinguish stronger odors with different qualities when all stronger odors are represented by silent response patterns. In contrast to the first experiment, the little response of LN2 shows that the global inhibition is not involved in the concentration-invariant coding (Fig. $3.4 \mathrm{~F}$ ).

Finally, we investigate the effect when both local inhibition and global inhibition are intensified. In this experiment, we raise the weights of partially-connecting LN1 to 3.2 (the same as the second experiment in this section) and the weights of fullyconnecting LN2 to 0.8 (the same as the third experiment). Similar to the results in the second experiment (Fig. 3.5A), the antennal lobe decorrelates different odor 

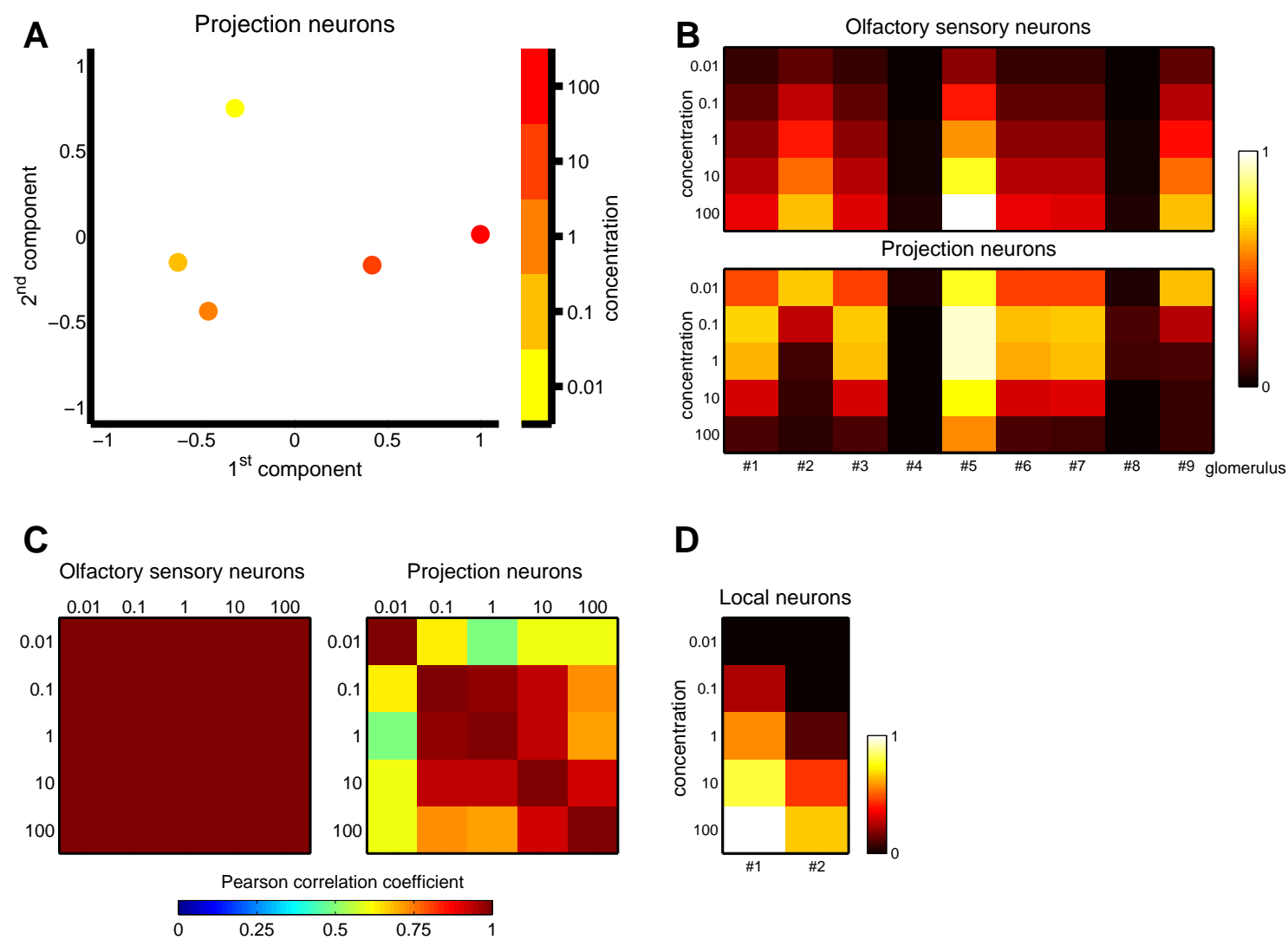

D

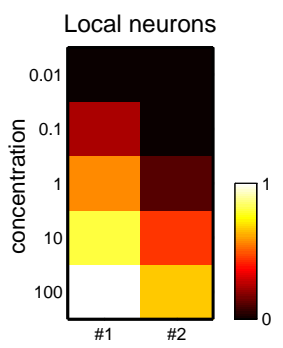

Figure 3.7: The antennal lobe generalizes odors at concentrations from 0.1 to 100 , but the mechanisms of concentration invariance are attenuated when the weights of the partially-connecting LN and the fully-connecting LN are increased from 2.4 and 0.2 in Fig. 3.4 respectively. Lateral connection weights: $W_{L N 1}=3.2 ; W_{L N 3}=0.8$. A. PN responses in the PCA space. B. Response matrices of OSNs and PNs. C. Correlation matrices representing the pairwise similarity between OSN responses and between PN responses to different concentrations. D. Response matrix of LNs. The activation of LN2 provides feedback global inhibition. Odors at concentrations from 0.1 to 100 are generalized because the weights of the partially-connecting LN increase from 0.2 to 0.8 , but the mechanism of concentration invariance is weakened by the global inhibition. Plot representations are the same as Fig. 3.4.

concentrations and generalizes odors at concentrations from 0.1 to 100 (Fig. 3.7A). However, the increased global inhibition implicitly attenuates the mechanisms of concentration invariance, and the output representations in the stronger odor cluster more spread out than the output representations categorized into the stronger odor cluster in the second experiment (comparing Fig. 3.7A with Fig. 3.5A). The correlation coefficients within the stronger odor cluster in Fig. 3.7C lower than those within the stronger odor cluster in Fig. 3.5C also indicate that the mechanisms of 
generalizing higher concentrations are undermined. On the basis of all above experiments in this section, we can conclude that global inhibition should not be the candidate for achieving the concentration-invariant coding.

\subsubsection{Empirical OSN response profiles}

Table 3.3: OSN responses to odor B in each glomerulus and LN lateral connections to glomeruli.

\begin{tabular}{cccccccccc}
\hline Glomerulus No. & 1 & 2 & 3 & 4 & 5 & 6 & 7 & 8 & 9 \\
\hline \hline $\begin{array}{c}\text { OSN response } \\
\text { profile to odor B }^{a}\end{array}$ & V & III & IV & I & IV & V & IV & III & II \\
\hline $\begin{array}{c}\text { LN1 connection }^{b} \\
\quad(\text { weight:2.4) }\end{array}$ & & $\mathrm{x}$ & & $\mathrm{x}$ & & & & & $\mathrm{x}$ \\
$\begin{array}{c}\text { LN2 connection } \\
\text { (weight:0.2) }\end{array}$ & $\mathrm{x}$ & $\mathrm{x}$ & $\mathrm{x}$ & $\mathrm{x}$ & $\mathrm{x}$ & $\mathrm{x}$ & $\mathrm{x}$ & $\mathrm{x}$ & $\mathrm{x}$ \\
\hline
\end{tabular}

${ }^{a}$ Nine glomeruli have individual OSN types with five different response profiles (I to V) to odor B. The details of response profiles over concentrations for each OSN types are depicted in Fig. 3.8A.

$b$ The " $\mathrm{x}$ " means the connection between the LN and this glomerulus.

In the following experiments, we change the OSN response profiles from a simplified linear relationship with logarithmic concentration to more realistic responses to concentration. On the basis of several neurophysiological data $[10,39,75,90,105,109$, $112,130,132]$, the response profiles of OSNs are generalized to have the following features:

1. Not each OSN type with the same odor preference has the same dynamic range. The dynamic range of high-affinity OSNs spans mostly over lower concentrations (e.g. OSNs in glomerulus No. 4 in Fig. 3.8A) whereas low-affinity OSNs are more active at higher concentrations (e.g. OSNs in glomerulus No. 9 in Fig. 3.8A).

2. Except for the OSN type preferring a given odor, other OSN types should respond very little at low concentration and respond more at very high concentration because the olfactory receptors are strongly stimulated.

3. Some OSN response profiles can be described as a linear relationship with logarithmic concentration, and some profiles can be fitted to a logistic function. 
A
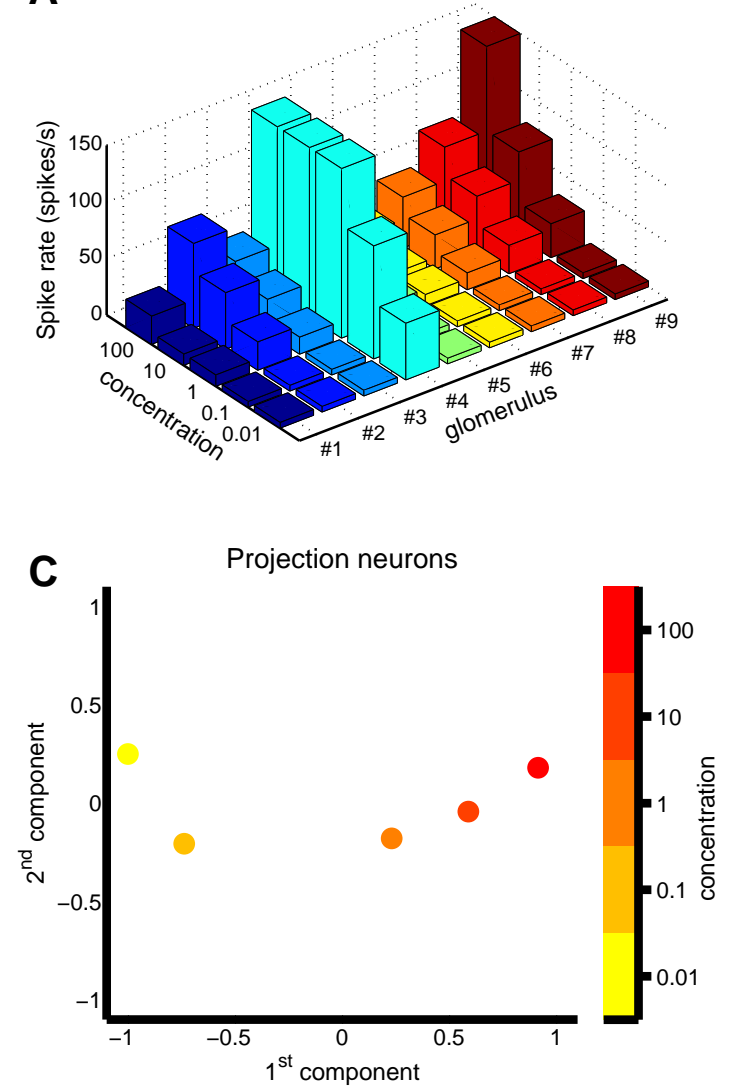

E

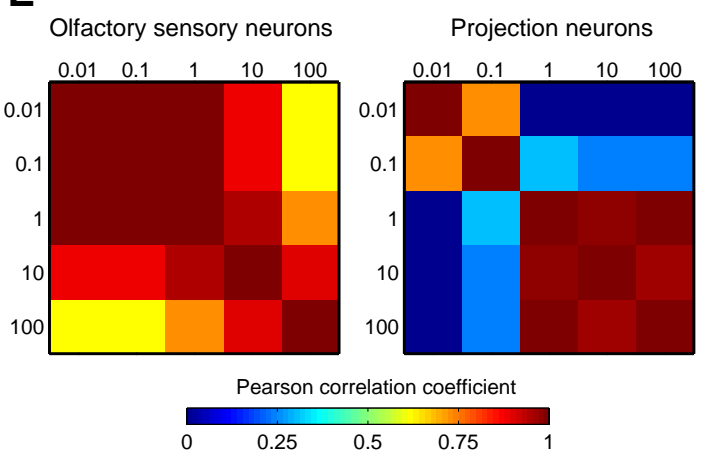

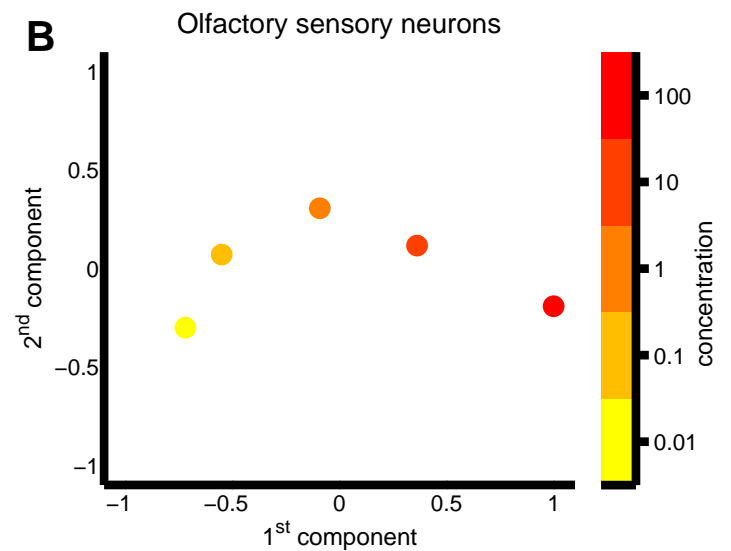

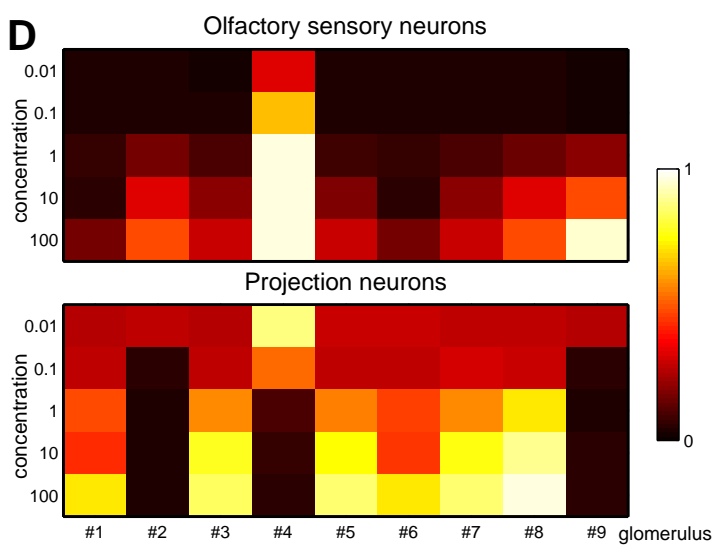

$\mathbf{F}$

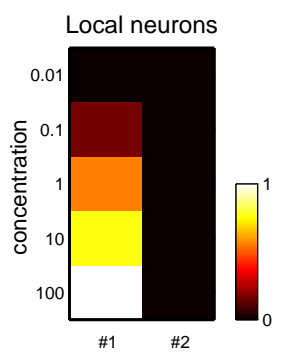

Figure 3.8: Neuronal responses to odor B of which response profiles are based on empirical data. Although a different odor is applied, the neural circuits with the same recurrent connectivity as earlier experiments still perform concentrationinvariant coding and separate odors into two clusters at the output level. Lateral connection weights: $W_{L N 1}=2.4 ; W_{L N 2}=0.2$. A. Response profiles for each OSN type. B. OSN responses in the PCA space. C. PN responses in the PCA space explaining $97.7 \%$ variance of original data. D. Response matrices of OSNs and PNs. E. Correlation matrices representing the pairwise similarity between OSN responses and between PN responses to different concentrations. F. Response matrix of LNs. Plot representations are the same as Fig. 3.4. 
Nine OSN types with five different response profiles to odor B are listed in Table 3.3, and their individual responses to concentrations are depicted in Fig. 3.8A. All parameters in the model and connectivity patterns we use for this experiment are the same as the first experiment in subsection 3.4.2. Without changing the connectivity of lateral inhibition, the same antennal lobe network still performs concentrationinvariant coding and separates different concentrations into two clusters at the output level (Fig. 3.8). The results do not mean that this particular lateral connectivity can always successfully perform these olfactory strategies for all odors evoking diverse odor-evoked OSN patterns, but indicate that each lateral connectivity does not function only for encoding one particular odor. The neural mechanisms shown in previous experiments for categorizing odors with the same quality into lower and higher concentration clusters still appear here. For example, an abrupt transition of PN responses in glomerulus No. 4 occurs when the odor concentration increases from 0.1 to 1 (Fig. 3.8D).

In earlier experiments, the odor-evoked OSN patterns across different concentrations are highly correlated. Here the correlation of OSN activation patterns between the lowest two and the highest concentration is not close to 1 but around 0.7 (Fig. 3.8E). This is because some OSN types respond little to lower concentrations, but very strong stimulus can activate them. Therefore, these results of correlation are in concert with the second feature of presumed OSN response profiles.

For getting better results, we slightly modify the previously used connectivity pattern in Table 3.3. First, we set LN1 to connect one more glomerulus No. 3. Then we slightly reduce the weights of partially-connecting LN1 from 2.4 to 2, and raise the weights of fully-connecting LN2 from 0.2 to 0.32 . The results of output representations after we slightly modified the lateral connectivity are shown in Fig. 3.9. The distances between output representations within the lower concentration cluster decrease in the PCA space (Fig. 3.9A), and the odor generalization is improved. Another enhancement is that the correlation between output representations within clusters increases, and the correlation between output representations across clusters decreases (Fig. 3.9C).

The results suggest that manipulating the connectivity patterns of recurrent networks can cause quantitative change of clustering and enhance the separation between clusters at the output level. Therefore, different species can develop their individual inhibitory spatial connectivity to meet specific requirements such as encoding specific odors related to their food or predators with more separable representations between different concentrations via evolutionary and ecological processes.

Next, we show that weak global inhibition can be of benefit to the olfactory coding. We slightly raise the weights of all-connecting LN2 from 0.32 to 0.48 . On the basis of new connectivity, only the odor at the highest concentration can activate LN2 (Fig. 3.10D). In the PCA space, the variance of output representations in the 

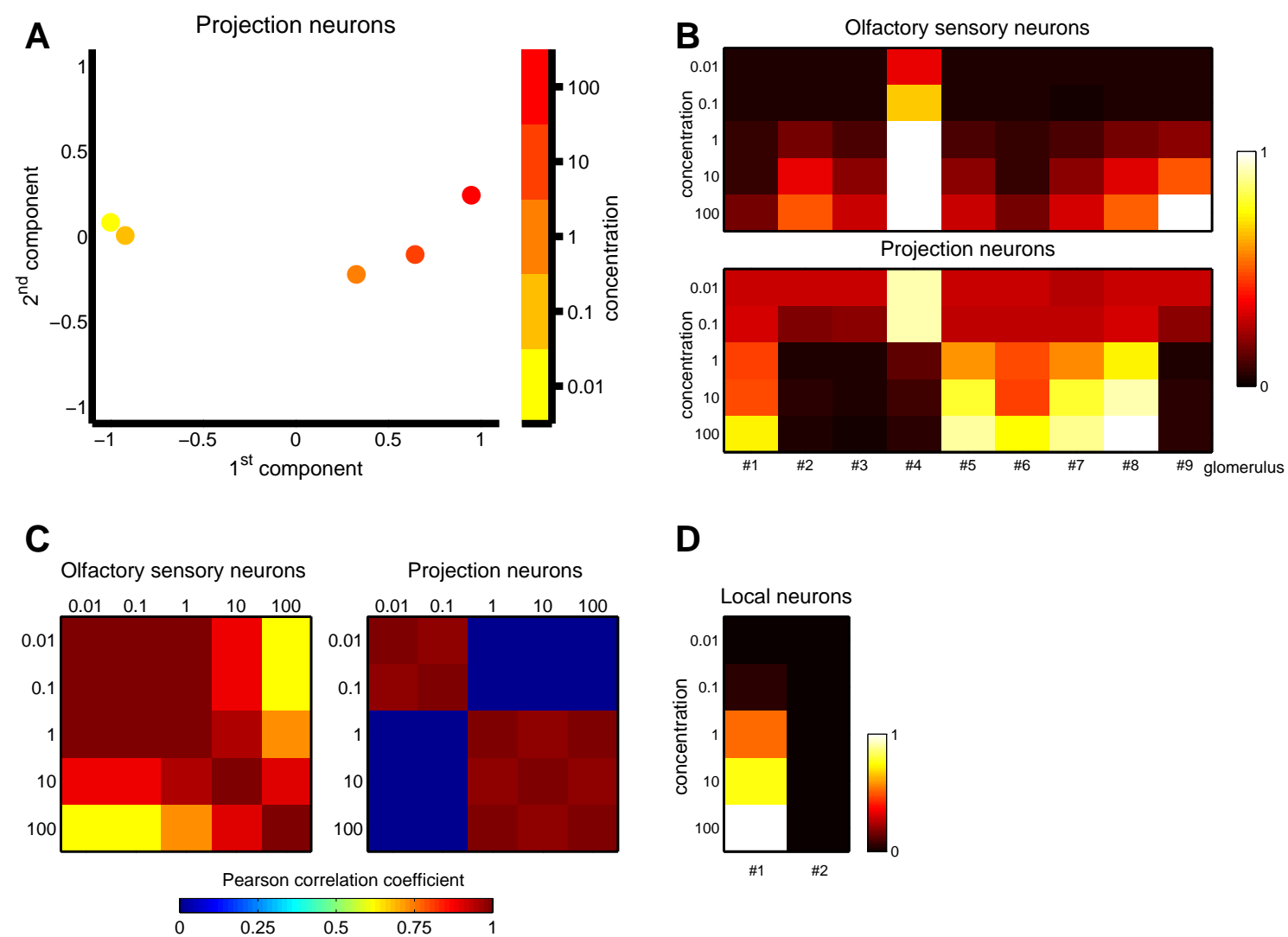

D

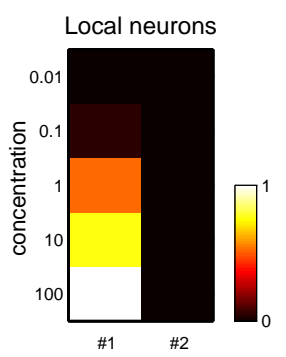

Figure 3.9: Neuronal responses in the antennal lobe to odor B when LN connectivity is modified to get better results. Improvement includes the distances between output representations within the lower concentration cluster decreasing in the PCA space. Another enhancement is that the correlation between output representations within clusters increases, and the correlation between output representations across clusters decreases. Lateral connection weights: $W_{L N 1}=2 ; W_{L N 2}=0.32$. A. PN responses in the PCA space explaining $98.5 \%$ variance of original data.. B. Response matrices of OSNs and PNs. C. Correlation matrices representing the pairwise similarity between OSN responses and between PN responses to different concentrations. D. Response matrix of LNs. Plot representations are the same as Fig. 3.4.

higher concentration cluster becomes less than that in the last experiment (comparing Fig 3.9A with Fig 3.10A). The reason why the point representing the highest concentrations moves closer to other points in the same cluster in the PCA space is that the global inhibition suppresses the redundant responses evoked by the very strong stimulus. Although some odors are not preferred by some OSN types, they can actually activate these OSNs with very strong intensity. These OSNs may be not highly activated by very strong stimuli, but these odor-evoked signals can be intensified by nonlinear intraglomerular transformation. In this case, if the global 

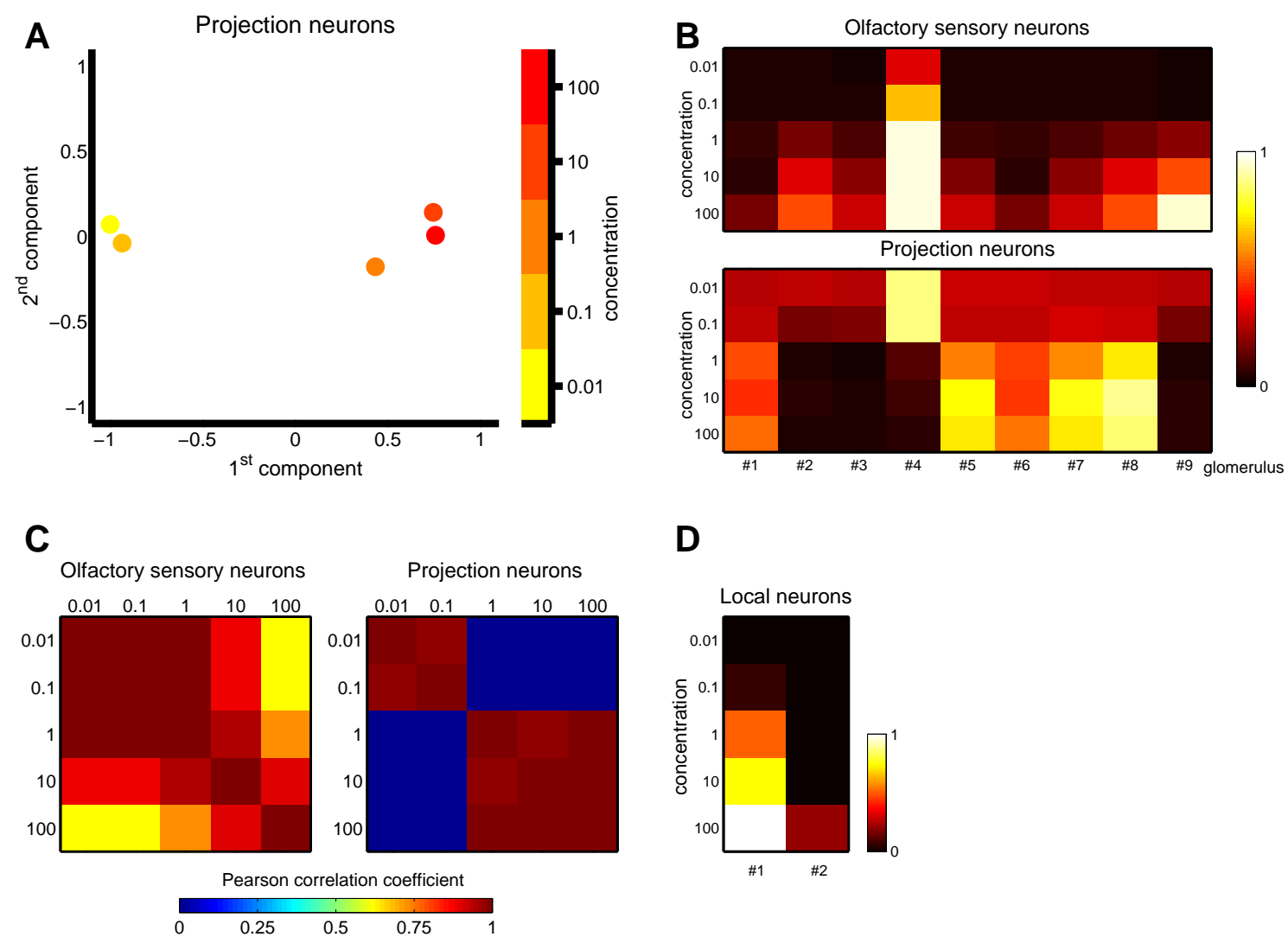

D

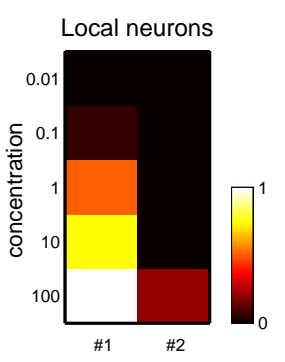

Figure 3.10: Neuronal responses in the antennal lobe to odor B when weak global inhibition at the highest concentration is recruited. The variance of the cluster generalizing higher concentrations becomes lower because the global inhibition suppresses the redundant responses evoked by the very strong stimulus. Lateral connection weights: $W_{L N 1}=2 ; W_{L N 2}=0.48$. A. PN responses in the PCA space explaining $98.9 \%$ variance of original data. B. Response matrices of OSNs and PNs. C. Correlation matrices representing the pairwise similarity between OSN responses and between PN responses to different concentrations. D. Response matrix of LNs. Plot representations are the same as Fig. 3.4.

inhibition is weak and only occurs at very high concentration, such inhibition should counterbalance these intensified odor-evoked signals. Therefore, the PN responses at concentration 100 are much similar to the PN responses at concentration 10 when the weak global inhibition is involved (comparing Fig 3.9B with Fig 3.10B).

In brief, in this subsection we have shown the results when our model applies to more realistic OSN response profiles, how the resulting representations change given different connectivity, and the explanation why the weak global inhibition can help the concentration-invariant coding. 


\subsubsection{Experiment: two odor qualities and more inhibitory LNs}

Table 3.4: OSN responses to odor $\mathrm{B}$ and $\mathrm{C}$ in each glomerulus and LN lateral connections to glomeruli.

\begin{tabular}{cccccccccc}
\hline Glomerulus No. & 1 & 2 & 3 & 4 & 5 & 6 & 7 & 8 & 9 \\
\hline $\begin{array}{c}\text { OSN response } \\
\text { profile to odor B } \\
\text { OSN response }\end{array}$ & V & III & IV & I & IV & V & IV & III & II \\
$\begin{array}{c}\text { OSofile to odor C } \\
\text { pro }\end{array}$ & V & IV & I & IV & V & II & III & IV & III \\
\hline $\begin{array}{c}\text { LN1 connection } \\
\quad(\text { weight:2) }\end{array}$ & & $\mathrm{x}$ & $\mathrm{x}$ & $\mathrm{x}$ & & & & & $\mathrm{x}$ \\
$\begin{array}{c}\text { LN2 connection } \\
\quad(\text { weight:2) }\end{array}$ & $\mathrm{x}$ & & $\mathrm{x}$ & & & $\mathrm{x}$ & & & \\
$\begin{array}{c}\text { LN3 connection } \\
(\text { weight:0.48) }\end{array}$ & $\mathrm{x}$ & $\mathrm{x}$ & $\mathrm{x}$ & $\mathrm{x}$ & $\mathrm{x}$ & $\mathrm{x}$ & $\mathrm{x}$ & $\mathrm{x}$ & $\mathrm{x}$ \\
\hline
\end{tabular}

${ }^{a}$ Nine glomeruli have individual OSN types with five different response profiles (I to V) to odor $\mathrm{B}$ and $\mathrm{C}$. The details of response profiles to odor B and $\mathrm{C}$ for each OSN types are depicted in Fig. 3.8A and Fig. $3.12 \mathrm{~A}$ respectively.

${ }^{b}$ The " $\mathrm{x}$ " means the connection between the LN and this glomerulus.

Now we extend the recurrent networks of the antennal lobe to include one more partially-connecting LN of which connectivity different from the previous LN1 and measure PN responses to a new odor C. The lateral connectivity of three LNs and the OSN response profiles to odor $\mathrm{C}$ are listed in Table 3.4. We implement recurrent networks incorporating more complicated lateral inhibition and investigate the effect of such connectivity on the output responses of antennal lobe network to different input combinatorial codes.

The experimental results show that given the same odor B we previously used in subsection 3.4.4, separating and generalizing odor representations still emerge in different connectivity at the output level (comparing Fig. 3.11 with Fig. 3.10) although one more LN is included in recurrent networks. No responses in LN2 (Fig 3.11D) implies that activation of LNs is specific to odors. Odors evoke combinatorial OSN responses in specific glomeruli, and only the LNs innervating to those activated glomeruli are possibly evoked by these odors. Moreover, how much overlap between the activated glomeruli and the glomeruli LNs innervate, and how strong the odor signals sent to the innervated glomeruli from their presynaptic OSNs are also the decisive factors for activating particular LNs. Therefore, the activation of each LN is specific to respective odors, and the feedback inhibition from each LNs is also specific to odors. 

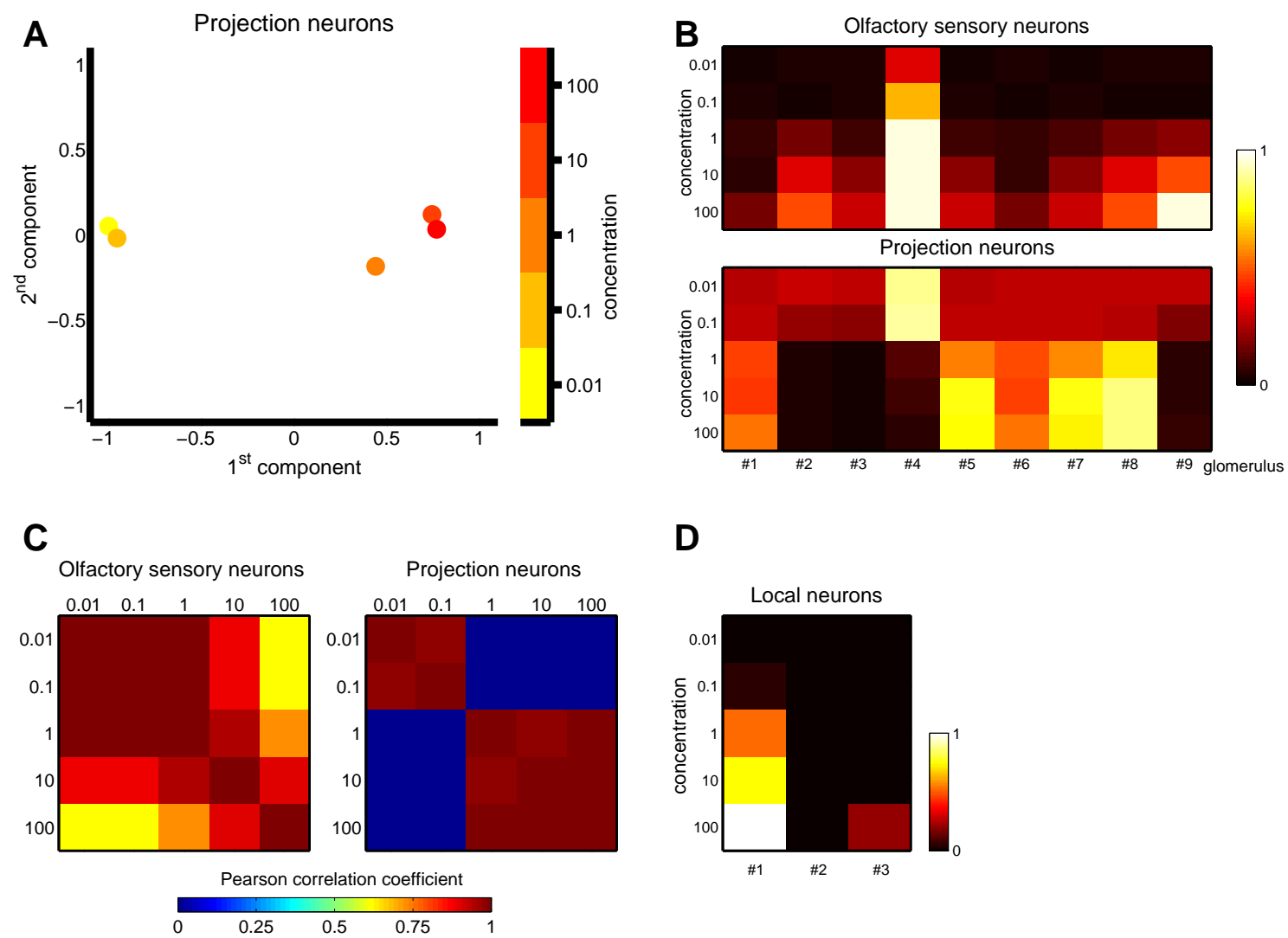

D

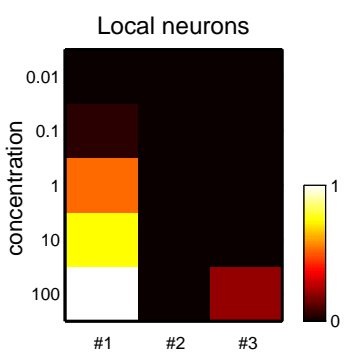

Figure 3.11: Neuronal responses in the antennal lobe to odor B when more complicated lateral connectivity is applied. Separating and generalizing odor representations still emerge in different connectivity at the output level although one more LN is included in recurrent networks. Lateral connection weights: $W_{L N 1}=2 ; W_{L N 2}=2$; $W_{L N 3}=0.48$. A. PN responses in the PCA space explaining $99.2 \%$ variance of original data. B. Response matrices of OSNs and PNs. C. Correlation matrices representing the pairwise similarity between OSN responses and between PN responses to different concentrations. D. Response matrix of LNs. Plot representations are the same as Fig. 3.4.

These results may answer the question: are PN responses still the same when more LNs are added to the recurrent network of the antennal lobe? Because LNs selectively respond to odors, if the added LNs do not respond to the odor stimulus as LN2 in this experiment, no additional lateral inhibition will be recruited, and the PN response patterns will be intact. There are about one hundred LNs in the antennal lobe of Drosophila, and less than 40 percent of LNs are featured by glomerulusspecific innervation [32]. The 50 glomeruli in Drosophila should allow 30 40 LNs to establish characteristic innervation patterns and to respond to specific odors.

In another experiment, the neuron response patterns evoked by odor $\mathrm{C}$ are shown 
A
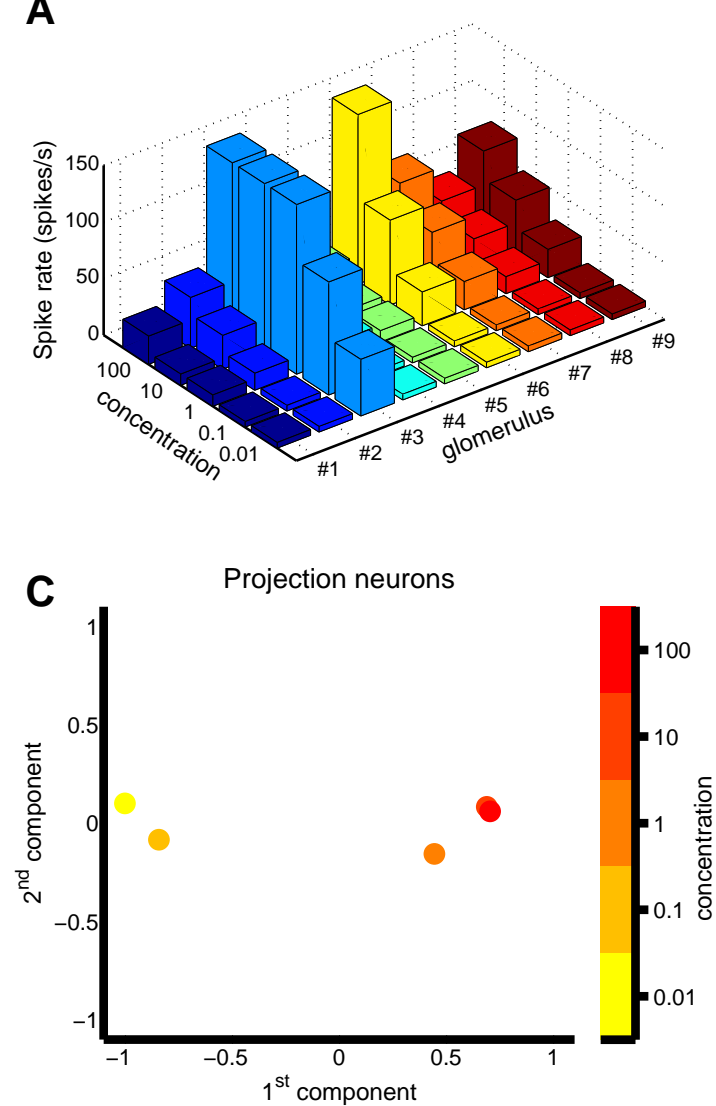

E

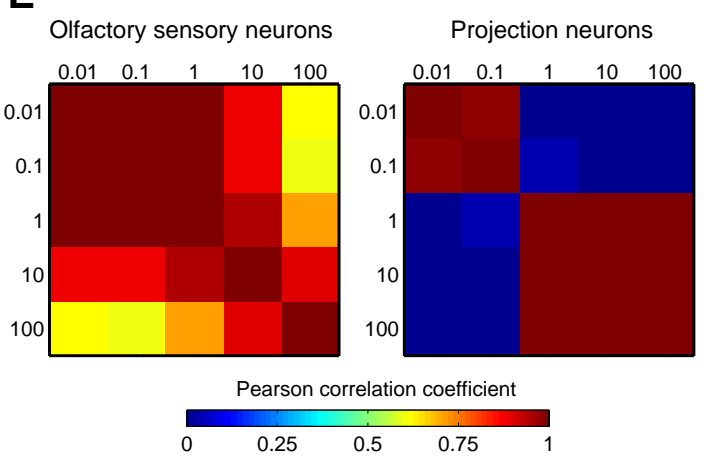

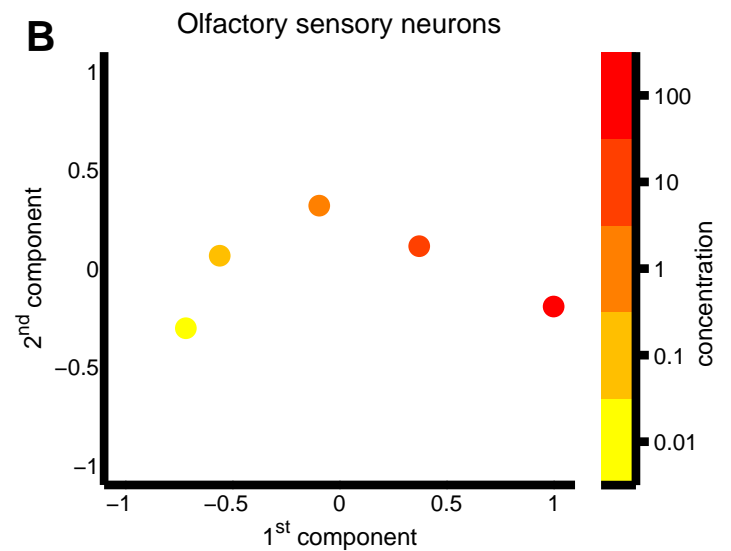

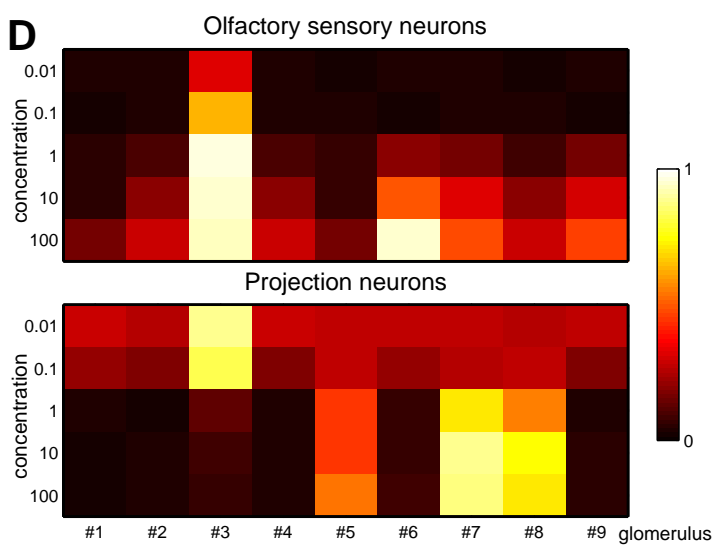

$\mathbf{F}$

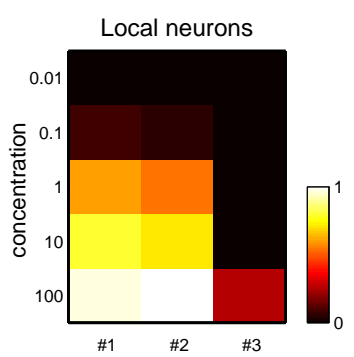

Figure 3.12: Neuronal responses in the antennal lobe to odor $\mathrm{C}$ when complicated lateral connectivity is applied. The same recurrent networks can still separate odor $\mathrm{C}$ at different concentrations into two clusters representing weak and strong odors at the output level. Lateral connection weights: $W_{L N 1}=2 ; W_{L N 2}=2 ; W_{L N 3}=0.48$. A. Response profiles for each OSN type. B. OSN responses in the PCA space. C. PN responses in the PCA space explaining $99.3 \%$ variance of original data. D. Response matrices of OSNs and PNs. E. Correlation matrices representing the pairwise similarity between OSN responses and between PN responses to different concentrations. F. Response matrix of LNs. Plot representations are the same as Fig. 3.4. 

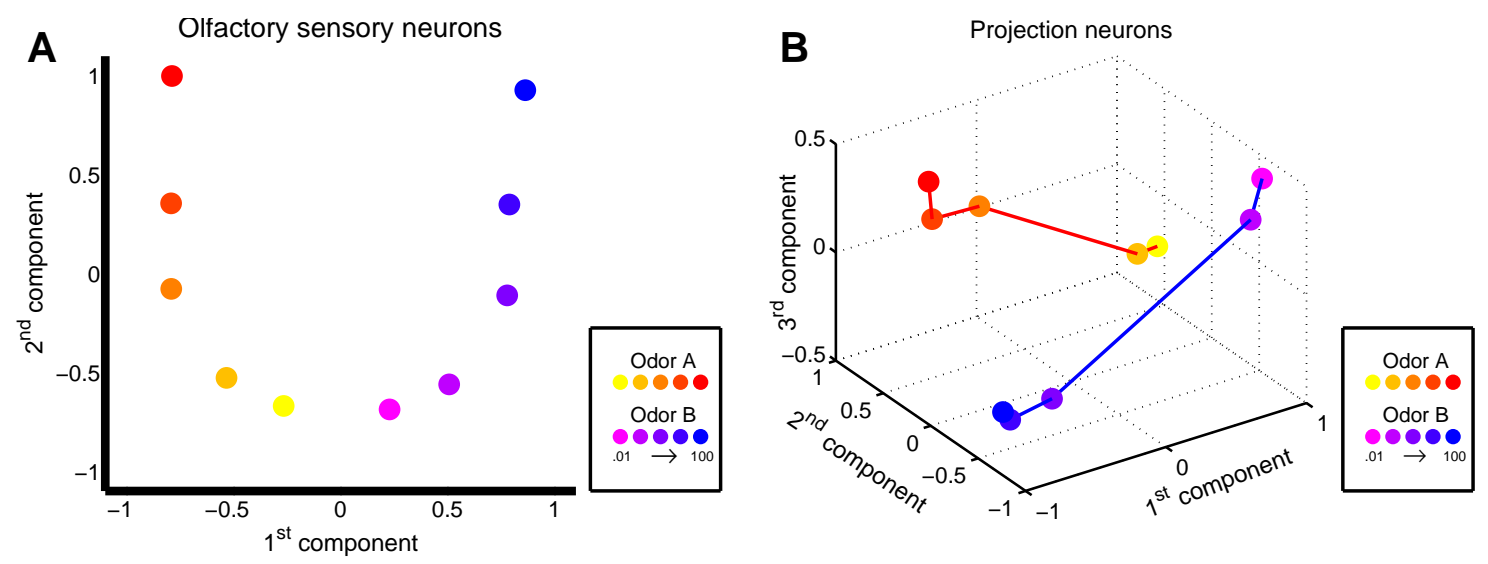

Figure 3.13: The response patterns of odor $\mathrm{B}$ and odor $\mathrm{C}$ are jointly represented in the same PCA space. A. The response patterns of odor B and odor $\mathrm{C}$ at the OSN level in the PCA space. Two color gradients represent a series of different concentrations for odor $\mathrm{B}$ and $\mathrm{C}$, respectively. The distance between odor $\mathrm{B}$ and odor $\mathrm{C}$ becomes more separated when odor concentration is increasing. $\mathrm{B}$. The response patterns of odor $\mathrm{B}$ and odor $\mathrm{C}$ at the $\mathrm{PN}$ level in the PCA space explaining 98.5\% variance of original data. The recurrent networks can distinguish odor quality, as well as categorizing odor quantity.

in Fig 3.12. The odor $\mathrm{C}$ across different concentrations can be still encoded into concentration-invariant representations although OSN response patterns evoked by odor $\mathrm{C}$ are different from those evoked by odor B. At the output level, the identical recurrent networks separate odor $\mathrm{C}$ at different concentrations into two clusters representing weak and strong odors. This may suggest that one set of lateral inhibition can perform the same olfactory strategies, concentration-invariant coding and intensity categorization, to different odor qualities.

Surprisingly, both two partially-connecting LNs are involved in the olfactory processing of odor C (Fig. 3.12F). Although the lateral connectivity of LN1 and LN2 differ, they respond similarly across concentrations. Their similar responses result from that both LNs connect to glomerulus No 3 where the OSN type has high affinity for odor C. Strong odor-evoked signals activate both LNs through glomerulus No. 3 so that both LNs are simultaneously recruited in this olfactory processing. Both LNs are guided by the same activating signals, and they coordinate to inhibit PN response (Fig 3.12D). So it is possible that the concentration-invariant coding and odor generalization can be achieved by several coactive LNs. On the basis of the last two experiment results, we point out that not only LN connectivity but input odor-evoked patterns can contribute to the selectivity of LN activation.

Finally, the response patterns of odor $\mathrm{B}$ and odor $\mathrm{C}$ are jointly represented in the same PCA space (Fig. 3.13). The distances between the OSN response patterns at lowest concentration are closer than those at higher concentrations in the PCA space 
Table 3.5: Summary of experiments in this chapter.

\begin{tabular}{cccc}
\hline Figure & Odor stimulus $^{a}$ & $W_{L N 1}{ }^{b}$ & $W_{L N 2}{ }^{b}$ \\
\hline \hline Fig. 3.4 & odor A & 2.4 & 0.2 \\
Fig. 3.5 & odor A & 3.2 & 0.2 \\
Fig. 3.6 & odor A & 2.4 & 0.8 \\
Fig. 3.7 & odor A & 3.2 & 0.8 \\
Fig. 3.8 & odor B & 2.4 & 0.2 \\
Fig. 3.9 & odor B & 2 & 0.32 \\
Fig. 3.10 & odor B & 2 & 0.48 \\
\hline
\end{tabular}

${ }^{a}$ The OSN response to odor A increases linearly with concentration in logarithmic scale. The OSN response to odor B is based on the rules generalized from empirical data.

${ }^{b} W_{L N 1}$ and $W_{L N 2}$ are the weights of partially-connecting LN1 and fullyconnecting LN2 respectively.

\begin{tabular}{ccccc}
\hline Figure & Odor stimulus $^{a}$ & $W_{L N 1}{ }^{b}$ & $W_{L N 2}{ }^{b}$ & $W_{L N 3}{ }^{b}$ \\
\hline \hline Fig. 3.11 & odor B & 2 & 2 & 0.48 \\
Fig. 3.12 & odor C & 2 & 2 & 0.48 \\
\hline
\end{tabular}

${ }^{a}$ The OSN response to odor B and odor $\mathrm{C}$ is based on the rules generalized from empirical data.

${ }^{b} W_{L N 1}$ and $W_{L N 2}$ are the weights of partially-connecting LN1 and LN2 respectively, and $W_{L N 3}$ is the weight of fully-connecting LN3.

(Fig. 3.13A). Only a few OSN types with high affinity can respond to odor stimuli at very low concentration meaning that merely a little amount of odor information can be used to differentiate odor quality. More OSN types are progressively activated as odor concentration is being raised. This causes odor representations more distinct and separated between odor $\mathrm{B}$ and odor $\mathrm{C}$ at the same concentrations when more OSN types exhibit differential responses to these two odors.

Invariant concentration representations at the $\mathrm{PN}$ level for both odor $\mathrm{B}$ and odor $\mathrm{C}$ are revealed in the PCA results (Fig. 3.13B) when both odors are reduced their dimensionality on the same linear transformation basis. For each odor, the olfactory processing categorizes their representations into weak and strong groups, respectively. While the recurrent networks perform the mechanisms of concentration invariance and odor intensity categorization into two clusters, each output representation still retains discriminable information for representing its corresponding odor 
identity in the antennal lobe. Therefore, our results show that this kind of recurrent networks can distinguish odor quality, as well as categorizing odor quantity.

The neuron response patterns discretely represent distinct quantity as these patterns encode different quality. When the representations of odor B and odor C are separated at the PN level, the higher concentrations are also segregated from the lower concentrations in the same odor. These clusters are situated at different locations in the odor space represented by PN responses. The evidence gathered in our experiments confirms the conclusions of several behavioral studies, suggesting that different quantity may be encoded as different quality in the olfactory processing.

The experiments demonstrated in this chapter are summarized in Table 3.5 for comparing the results in different conditions.

\subsection{Discussion}

The olfactory systems across different species can perform conflicting strategies in the same neuronal circuits simultaneously: being sensitive to intermediate concentration change and robust against small concentration fluctuation. Our simulation results provide evidence that the local lateral inhibition can primarily serve to perform these two functions in the same recurrent networks. The effect of lateral inhibition can suppress PN responses at higher concentrations and separate them from those at lower concentrations. Therefore, the same odors at different concentrations are generalized into to respective clusters representing weak and strong odors. The lateral inhibition also inhibits PN responses across higher concentrations and keeps odor representations independent of concentration fluctuation. Although we verified that global inhibition is not mainly responsible for the concentration-invariant coding, weak global inhibition can counterbalance the increasing responses of PNs at higher concentrations, thereby stabilizing odor representations. For other uninhibited PNs, the interglomerular nonlinear function boosts their responses into saturation area at higher concentrations and partly results in concentration-invariant representations. Overall, such sophisticated mechanisms within and between glomeruli can grant the olfactory system sensitivity power for encoding the quantity of odors and robustness against fluctuation.

It should be noted that the purpose of implementing the concentration-invariant coding in the olfactory system is not to collapse all quantitative information. Alternatively, olfactory networks still categorize odors into weak and strong signals depending on their concentration information. Notably, this mechanism helps the olfactory processing filter out the noise and concentration fluctuation unrelated to true odor intensity. 
The specificity of inhibition depends on both the spatial patterns of LN innervation and the combinatorial odor-evoked patterns of OSNs. In addition, the selectivity of LN inhibition depends on the quantitative patterns of input OSN responses because the activation of LN relies on sufficient input from OSNs through glomeruli. We already showed that one LN can achieve the olfactory encoding for specific odors, and the olfactory processing for one odor can be accomplished by several coactive LNs.

However, how many odors and which quality of odors can be encoded as concentrationinvariant representations given a set of lateral inhibition are not fully understood. We have not known yet the relationship between the strength of lateral connection and the threshold of input intensity to shift one representation from one cluster to the other. The bifurcation point where different concentrations are encoded into different output representations should reflect the odor significance for animals. For example, the antennal lobe should tag and categorize a significant odor as strong signals when the odor appears at average concentration. If only higher concentrations for this odor are categorized into strong signals, the higher brain centers may not notice the presence of this odor at average concentration when such average intensity is generalized with weak odors. To explore the parameter space of the recurrent networks in the antennal lobe can help us understand more about the olfactory system. To what extent these strategies depend on recurrent connectivity should be investigated in future work.

A report published recently indicated that at least six sequentially mechanisms including behavioral regulation and learning processing are required to achieve concentration invariance in the mammal olfactory system [124]. On the contrary, our model based on merely two inherent olfactory principles should be more capable of performing these conserved strategies across different species from the numerically complicated olfactory circuits in mice to the network marked by numerical simplicity in Drosophila.

In the antennal lobe of Drosophila, the numbers of OSNs and PNs are about 1300 and 150 respectively $[9,12,19,23,90,107]$. Another extreme ratio of input neurons to output neurons has been identified in honeybees where 64000 OSNs are located on each antenna projecting their axons to 800 PNs $[14,133,134]$. When the information streams from the relatively large amount of OSNs to much fewer PNs, it is unlikely for PNs as the output of the antennal lobe to process and encode each piece of information from OSNs. Therefore, in terms of channel capacity, the output of the antennal lobe (i.e. the processing channel) turns into the bottleneck of signal transmission in the olfactory system $[31,135]$ if we think the whole antennal lobe as a channel accommodating olfactory information flow. Interestingly, the findings in another insect (locusts) suggested that the mean spike rates of PNs are nearly constant and independent of concentration across different odors although the information streams from OSNs increase with concentration [76]. If the interspike intervals carry little information in the antennal lobe, the spike rate can be simply assumed as the 
amount of neural information. This assumption is likely to be true because the spike rate is highly related to odor intensity in OSN populations $[10,39,75,90,105,130,132]$. Therefore, the mean spike rates of $\mathrm{PN}$ populations varying little across concentrations just reflect the concentration-invariant representations in terms of information amount.

Another idea is that the local lateral inhibition also engages in information compression in the antennal lobe. An odor information usually consists of several streams flowing from OSNs to PNs in respective glomeruli. The local inhibition can redistribute the flow of streams among glomeruli and control their access to intraglomerular output. At low concentration, the amount of odor information is not high, and it is not necessary for applying information compression to the antennal lobe. The increase of concentration causes the amount of olfactory information raised. Part of this information increase results from the OSN types with low-affinity starting to respond from intermediate concentration. Therefore, more glomeruli are used and new streams are created. Because selective inhibition is also activated by the increase of concentration, part of glomeruli may stop transmitting or reduce the amount of transmitting information streams. Some channel capacity is then released from inhibited glomeruli and can be transferred to other uninhibited glomeruli. If the OSNs types with low-affinity project onto these uninhibited glomeruli, the information streams sent from these OSN types can be transmitted via the released channel capacity without largely increasing the usage of whole channel capacity. Such mechanisms may also reduce the neuronal energy to transmit olfactory information. Briefly, lateral local inhibition can distribute channel capacity among glomeruli to compress olfactory information at the PN level.

Take the experiment results in Fig. 3.10 as an example. At lower concentrations from 0.01 to 0.1, PNs in the glomeruli No. 4 spend much more channel capacity than PNs in other glomeruli (the lower panel in Fig. 3.10B) whereas the channel capacity is relocated to glomeruli No. 1, and No. 5 8 at higher concentrations from 1 to 100 . Therefore, relying on local inhibition the antennal lobe efficiently distributes the finite channel capacity among glomeruli across different concentrations.

In this chapter, we study how the neural circuits perform the olfactory strategies by the dynamics of neuronal interactions between different layers. The olfactory system can filter out the noise from concentration fluctuation and focus on the effect of quantitative change. In the environment, odors are also easily contaminated by chemical noise. Therefore, the olfactory system faces another challenge of identifying the odor quality when multiple odor qualities are mixed. We will move to this issue how the olfactory system recognizes odor quality change and represents the qualitative information of odor mixtures in the next chapter. 


\section{Discrete representations of odor mixtures}

\subsection{Overview}

We have previously investigated how the olfactory system processes odors different at concentrations but the same in quality. In this chapter, we turn to ask how the odor information of multiple qualities is processed in the olfactory system. First, we review recent studies of individual neuron responses to odor mixtures at the OSN and PN layers and investigate possible interactions between these two layers via recurrent networks. Then we continue to extend our view from the responses of individual neurons to the collective representation of neuron ensembles. Finally, we implement our model to interpret how the neural circuits generate clustered representations at the output level and why the number of clusters generalizing these representations results from the similarity between odor qualities in an odor pair.

\subsection{Mixture odors in olfactory processing}

\subsubsection{Responses of OSNs and PNs to odor mixtures}

In the absence of lateral inhibition, the increase of OSN spike rates always raises the spike rates of the presynaptic PNs for every glomerulus before PN responses reach saturation. This is the case when only one odor quality is presented, and the difference between evoked spike rates only reflects the quantity change (i.e. concentration change) of odor stimulus. Next, we ask how olfactory neurons in the antennal lobe respond to and process odor mixtures blending multiple odor qualities.

Interestingly, the responses of olfactory neurons to odor mixtures are not merely the summation of their responses to constituent odors, but can be predicted by several possible interactions between components. The interactions in OSNs between constituent odors are generally defined in four categorizations: (1) additivity, if the 

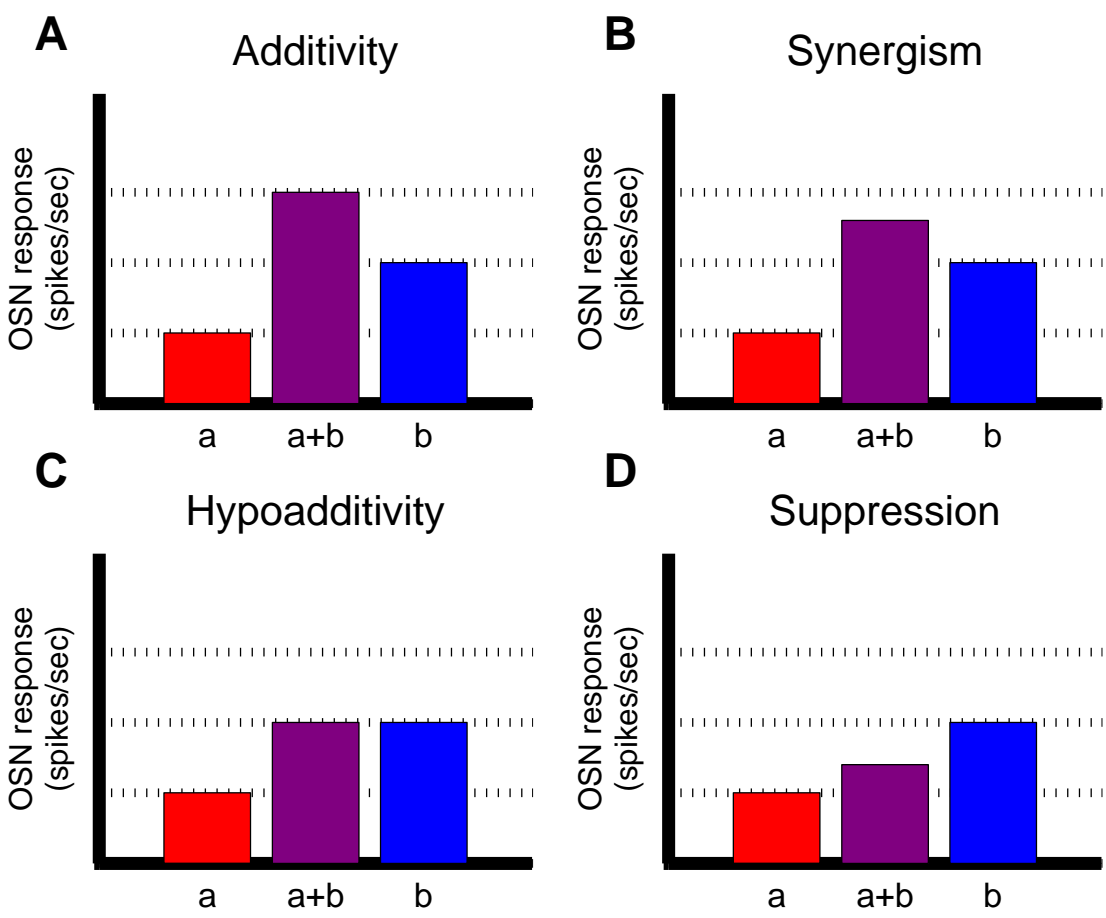

Figure 4.1: Schematic graphs depicting the responses to odor mixtures and their constituent odors in four interactions between components. The OSN responses to the odor mixture $(\mathrm{a}+\mathrm{b})$ and its constituent odors ( $\mathrm{a}$ and $\mathrm{b}$ ) are shown in purple, red, and blue bars, respectively. The maximum of responses to individual constituent odors is the response to odor $\mathrm{b}$ in each case. A. Additivity: the response to the odor mixture is equal to the sum of the respective responses to constituent odors. B. Synergism: the response to the odor mixture is less than the sum of the respective response to constituent odors but larger than the maximum of the responses to individual constituent odors. C. Hypoadditivity: the response to the odor mixture is close to the maximum of the responses to individual constituent odors. D. Suppression: the response to the odor mixture is much less than the maximum of the responses to individual constituent odors.

response to an odor mixture is equal to the sum of the respective responses to constituent odors alone (Fig 4.1A); (2) synergism, if the response to an odor mixture is less than the sum of the respective response to constituent odors but larger than the maximum of the responses to individual constituent odors (Fig 4.1B); (3) hypoadditivity, if the response to an odor mixture is close to the maximum of the responses to individual constituent odors, and other less efficient or weaker components are inactive (Fig 4.1C); (4) suppression, if the response to an odor mixture is much less than the maximum of the responses to individual constituent odors, and other components attenuate the effect of the most efficient or the strongest component (Fig 4.1D) [136, 137]. 
It has been revealed in recent years that the ways of interactions in the responses to odor mixtures are distinct at the levels of OSNs and PNs. In Drosophila, most interactions between constituent odors in OSNs attribute to synergism or at least hypoadditivity [54]. It has been little observed in Drosophila that the responses to odor mixtures were less than the response to any components, and only two such cases of odor mixtures were found in the same one glomerulus. In contrast, the suppressed responses to odor mixtures are more commonly seen at the PN level in Drosophila. Such suppression interaction of constituent odors occurred in all glomeruli and appeared in at least two different odor mixtures for each glomerulus. Comparable results have been also reported in honeybees where most PNs exhibited suppression and hypoadditivity in the responses to odor mixtures, and almost no synergism were found in PNs [138]. The number of glomeruli where PN exhibited suppression responses was more than the number of glomeruli where OSN exhibited suppression responses when the same odor mixtures were represented. Besides, the interactions in hypoadditivity at the OSN level also occurred more frequently than that at the PN level in honeybees. Likewise, more than $75 \%$ of PNs probed in the moth study showed inhibitory or biphasic (i. e. a few spikes succeeded by a period of inhibitory hyperpolarization) responses to odor mixtures [139].

Similar interactions between components of odor mixtures have been observed in the OSNs and MCs of olfactory bulbs, respectively [136, 140,141]. In zebrafish, a model based on the sum of the response to the strongest component and a positive interaction terms can predict the responses to odor mixtures at OSNs consistent with the observations in experiments [140]. The results implicitly suggest that the interactions between constituent odors at the OSN level can be classified as synergism. In rats, nearly $60 \%$ of OSN responses to odor mixtures were categorized into hypoadditivity in terms of constituent interactions [136] whereas part of MC responses to odor mixtures displayed suppression [141].

OSN responses to mixtures could be synergism, hypoadditivity or suppression (in rare cases) depending on the interactions of constituent odors in OSNs. How constituent odors interact with each other in OSNs may result from their competition to bind the main or two overlapping receptor sites of olfactory receptors $[142,143]$. Another possible explanation of these interactions comes from noncompetitive effects reshaping the properties of the main binding site at receptors [142]. However, until now no general and deterministic rules have been found to predict the responses of olfactory neurons at different layers to odor mixtures even though we know the respective responses of olfactory neurons to individual components [138, 139, 142]. Moreover, different combinatorial codes are applied to represent each odor quality at the OSN level, but little is known about how the antennal lobe or the olfactory bulb processes the more complicated combinatorial codes evoked by odor mixtures in neural coding. These odor-evoked patterns are possibly composed of several combinatorial codes representing respective components. In this chapter, we will implement our proposed model to show how the olfactory system processes odor 
mixtures to perform particular olfactory strategies. Next, we will start from the neuron interaction in networks to show how the information of odor mixtures propagates.

\subsubsection{Processing odor mixtures in recurrent networks}

We have known that PN activity responding to odor stimuli can be increased with afferent OSN input if the increasing input results from the rise in a single odor quantity, and no lateral inhibition acts on PNs. Remarkably, PN responses are usually attenuated by the increase of afferent input when this increase is mediated by an odor stimulus incorporating new odor qualities [54,88]. Because the synapses between OSNs to PNs in the same glomerulus are excitatory and strong [12,42-44], the afferent OSN input is unlikely the reason for causing PNs inhibited. In the recurrent networks of the antennal lobe, the possible and only possible mechanisms to inhibit PNs at the output level should attribute to the lateral inhibition from LNs.

The interactions of constituent odors at the PN level could be suppression, hypoadditivity, or synergism. According to the experimental results from several recent studies, suppression occurred more frequently than synergism did in PNs [54, 138-141, 144]. In honeybees, the increase number of constituents in odor mixtures attenuates PN activity and causes more PN responses suppressed [137,138]. The number of suppression increased in PN responses is not related to odor quality but depends on the number of components.

The lateral inhibition should not be main effect in synergism because the neuron activity increases at both input and output levels. In contrast, the lateral inhibition may explain why the responses to odor mixtures are suppressed as compared with the responses to each individual components. Different odor quality can activate OSNs in different sets of glomeruli, and a mixture consisting of more odor qualities can activate OSNs in more glomeruli. The activation of LNs results from the input of OSN activity in glomeruli where these LNs laterally connect. The more glomeruli receive odor-evoked signals, the more LNs will be activated. Therefore, an odor mixture consisting of more components can activate more signals into glomeruli to elicit more inhibition from LNs, and the increase strength of inhibition causes more PNs inhibited to an odor mixture.

In Drosophila, more direct evidence was reported in using a receptor antagonist to block the inhibition onto PNs [54]. Without the effect of inhibition, the suppressed responses of PNs to odor mixtures change to synergism. The results suggested that the interactions of constituent odors at the PN level are related to the inhibition applied to PNs. Unlike the odor molecules of mixtures directly interacting in the site of olfactory receptors at the OSN level, the interactions between different odor 
qualities at the PN level do not actually take place within PNs but through the recurrent connections of networks. Briefly, the increase or decrease of PN responses to odor mixtures compared with the responses to odor components depends on the new responses of their cognate OSNs to odor mixtures and lateral inhibition if the inhibition is recruited in the neural computation.

On the grounds of results in Chapter 3, the heterogeneity of inhibition connectivity should also play a major role in processing odor mixtures. Indeed, suppression occurs in part of neurons and in part of odor mixtures indicating that the inhibition occurs specifically and locally, and such specific inhibition has been previously suggested in the PNs of Drosophila [54] and moths [139], and in the MCs of rats [141]. Therefore, after the computation in the antennal lobe or the olfactory bulb, the information encoding multiple qualities is represented by the neuronal responses with several interactions at the output level. Whether and how these olfactory representations distinct from those representing the single quality of constituent odors are dependent on the interactions between multiple molecules at receptors and the intra- and interglomerular computation.

\subsubsection{Representations of odor mixtures}

To only observe the responses of neurons in one glomerulus and at separate layers cannot help us comprehend the whole olfactory information processing. Now we turn to focus on odor response patterns across glomeruli. In honeybees, the odor representations of $\mathrm{PN}$ response patterns for odor mixtures are separated from those for odor components in the PCA space $[137,138]$. The representation of a binary mixture in the PCA space is not located on the straight line between two odor component representations, but away from that line to form a triangle together with odor component representations. The distances from the representation of a binary mixture to those of constituent odors are not always equal. In some odor mixtures, the representations are more close to one component than the other. The same separation between odor mixtures and their components has also been found in the PN responses of the antennal lobe in locust [144].

Similar observations have been reported in zebrafish where the mixture representations have same structural relationship with component representations in the PCA space [119]. Furthermore, two findings in their report particularly arouse our interest. First, this report suggested that although an odor was blended with another odor in relative low intensity (ratio: 99 to 1 ), the representation of this odor mixture was still generalized with the representation of a pure odor (ratio: 100 to 0 ) at the output level (Fig 4.2). When this ratio was decreased, the corresponding representation abruptly shifted to the cluster standing for odor mixtures. This abrupt transition also occurred again between 10/90 and 1/99, and the 1/99 mixtures and the $0 / 100$ pure odor were generalized. Notably, we have seen such generalization 


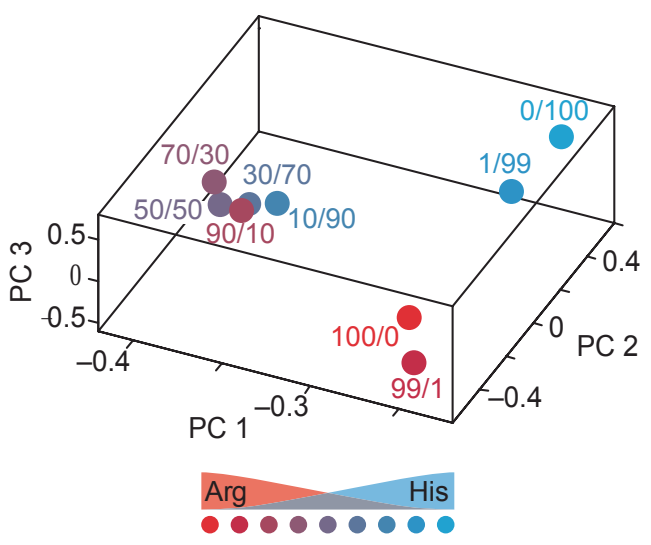

Figure 4.2: The representations in morphing between dissimilar odors projected onto the first three principal components. The neuronal responses were measured in the MCs of zebrafish. The representations of odor mixtures are separated from those of two components. The mixtures in concentration ratios 100/0 and 99/1 are generalized. The same generalization also occurs in ratios 0/100 and 1/99. Figure adapted by permission from Macmillan Publishers Ltd: Nature [119], copyright (2010)

and abrupt transition of representations in encoding different odor quantity. Second, the representations of intermediate odor mixtures were separated from each representation of components if the mixtures consisted of dissimilar odors (Fig 4.2). Surprisingly, all intermediate odor mixtures were generalized with one of components if odor components are similar.

The first finding in zebrafish raises the question: how does the olfactory system evaluate an odor consisting of multiple odor qualities as an odor blend or a pure odor contaminated by chemical noise that has to be filtered out? If an odor mixture is actually a major odor contaminated by other minor odors, how can olfactory neural networks generalize this odor mixture with its major odor? The second finding suggests that all odor mixtures and their components are encoded in different numbers of discrete outputs - three for dissimilar odors and two for similar odors. If the similarity of odor components determines the number of discrete clusters encoded in the olfactory space, how does the olfactory bulb or antennal lobe perform these features in neural circuits? In the next section, we will implement our proposed model to explain these phenomena and to show how the recurrent networks process odor information consisting of multiple odor qualities. 


\subsection{Results in theoretical investigations}

Although we reproduce the experimental results mainly observed in the vertebrates, we still use the antennal lobe as the simulation basis for the consistency of all theoretical experiments. Again, the fact of the conserved architecture between insects and vertebrates in the first processing center should allow us to make this arrangement.

\subsubsection{Model setup}

Now we will implement the proposed model to demonstrate how the antennal lobe generalizes odor mixtures and its components into a few discrete representations. We will also use different odor pairs consisting of dissimilar odors or similar odors as input to demonstrate how recurrent networks process multiple qualities to produce different cluster numbers. After that, we will compare the neuronal responses in our experiments with experimental data in zebrafish.

The electrophysiological parameters in the equations of our model are the same as earlier experiments. The numbers of OSNs and PNs are still 30 and 3 per glomeruli respectively. We still implement combinatorial codes that different odor stimuli evoke different OSN types in different responses. There are dozens of LNs in the antennal lobe, and LN activation is specific to odors because of their heterogeneous lateral connections. Therefore, the lateral connectivity should be implemented differently depending on the given odor-evoked patterns. Here, we emphasize again that any lateral connectivity of LNs is not unique for encoding one particular odor and only works for that odor as we showed in Chapter 3.

\subsubsection{Experiment: Odor morphing between dissimilar odors}

The first experiment is to demonstrate the olfactory processing in recurrent networks for the pair of dissimilar odor D and odor E. Again, the networks consist of nine glomeruli, and we have nine different types of OSNs. The odor D and odor E are dissimilar, meaning that their OSN odor-evoked patterns are distinct. Therefore, OSNs in glomeruli No. 1, 2, and 5 prefer odor D and respond strongly to odor D while OSNs in glomeruli No. 6, 7, and 9 prefer the odor E and are strongly activated by odor E. The spike rates of nine OSN types to these two odors are listed in Table 4.1, respectively. The same OSN types projecting to the same glomeruli relay the odor signals to their postsynaptic PNs in the same glomeruli.

Both LN1 and LN2 are partially-connecting LNs, and LN3 is a fully-connecting $\mathrm{LN}$. The connectivity is shown in Table 4.1 where " $\mathrm{x}$ " means a connection between 
Table 4.1: OSN responses to odor D and $\mathrm{E}$ in each glomerulus and LN lateral connections to glomeruli.

\begin{tabular}{|c|c|c|c|c|c|c|c|c|c|}
\hline Glomerulus No. & 1 & 2 & 3 & 4 & 5 & 6 & 7 & 8 & 9 \\
\hline $\begin{array}{l}\text { OSN responses to } \\
\text { odor } \mathrm{D}^{a}\end{array}$ & 100 & 150 & 50 & 5 & 100 & 5 & 50 & 5 & 5 \\
\hline $\begin{array}{l}\text { OSN response to } \\
\text { odor } \mathrm{E}^{a}\end{array}$ & 5 & 50 & 5 & 5 & 5 & 100 & 100 & 150 & 50 \\
\hline $\begin{array}{l}\text { LN1 connection }{ }^{b} \\
\quad\left(W_{L N 1}: 2.4\right)\end{array}$ & $\mathrm{x}$ & $\mathrm{x}$ & & $\mathrm{x}$ & & & & & \\
\hline $\begin{array}{l}\text { LN2 connection } \\
\\
\quad\left(W_{L N 2}: 2.4\right)\end{array}$ & & & & & & $\mathrm{x}$ & $\mathrm{x}$ & & $\mathrm{x}$ \\
\hline $\begin{array}{c}\text { LN3 connection }{ }^{b} \\
\quad\left(W_{L N 3}: 0.48\right)\end{array}$ & $\mathrm{x}$ & $\mathrm{x}$ & $\mathrm{x}$ & $\mathrm{x}$ & $\mathrm{x}$ & $\mathrm{x}$ & $\mathrm{x}$ & $\mathrm{x}$ & $\mathrm{x}$ \\
\hline
\end{tabular}

the LN in this row and the glomerulus in this column. The weights of partiallyconnecting LN1 and LN2 are represented as $W_{L N 1}$ and $W_{L N 2}$, and both of them are 2.4 while the weights of fully-connecting LN3 $W_{L N 3}$ are 0.48 . The LN1 laterally connects to glomeruli No. 1, 2, and 4, and part of its connectivity overlaps with the glomeruli where OSNs strongly respond to odor D (i.e. No. 1 and No. 2). Therefore, LN1 can be activated and should be involved in the olfactory processing of odor D. Similarly, the OSN patterns evoked by odor E can elicit the activation of LN2, and LN2 should play an important role in processing odor E within the recurrent networks.

It is likely to raise a question why we choose such specific connectivity overlapping with glomeruli where strong OSN responses occur. This is because the LN response is indeed specific to odors and is based on OSN odor-evoked patterns. For example, another LN4 innervating to glomeruli No.1, 10, and 11 would be irrelevant to this experiment because neither odor $\mathrm{D}$ nor odor $\mathrm{E}$ can activate this LN if we extend this recurrent networks to an actual antennal lobe consisting of 50 glomeruli in Drosophila, and OSNs in glomeruli No. 10 and No.11 do not respond to odor D or odor E. Therefore, LN4 is excluded by the low proximity between the connectivity of this LN and OSN response patterns evoked by odor D or odor E. Furthermore, in all experiments of this study we do not selectively take the LNs producing favorable results to support our model, but only show the LNs specifically responding to and being enlisted in given odors. In this case, even though we include LN4 in this recurrent networks, the experimental results will be the same as the results given 


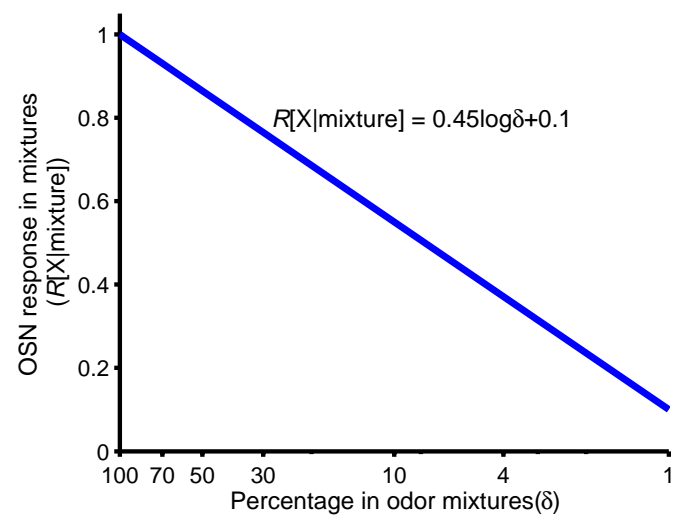

Figure 4.3: The spike rate of an OSN in response to an odor as the function of the percentage of this odor in mixtures. The linear relationship is governed by the equation indicated on the figure, and the percentage of this odor in mixtures is represented in a logarithmic scale. All responses of this OSN are normalized to its response to this odor representing at $100 \%$ in the mixture.

by the networks excluding LN4.

As earlier results, we represent neuronal responses with the spike rates averaged in the period of odor stimulation and visualize odor representations in a twodimensional space achieved by a linear dimensionality reduction PCA method. The concentration of components in odor mixtures varies in different ratios of one constituent odor to the other. OSN responses to an odor decrease when this odor is mixed and diluted with other odors. If the OSN response to a component odor $\mathrm{X}$ is expressed as $R[\mathrm{X}]$, we can formulate the relationship between the OSN response to the diluted odor $\mathrm{X} R[\mathrm{X} \mid$ mixture $]$ and the percentage of odor $\mathrm{X} \delta$ in this mixture by the following exponentially decreasing function:

$$
R[\mathrm{X} \mid \text { mixture }]=0.45 \times R[\mathrm{X}] \log \delta+0.1 .
$$

To prevent getting the response $R[\mathrm{X} \mid$ mixture $]$ less than zero from Eq. 4.1, we set the minimum value of $R[\mathrm{X} \mid$ mixture $]$ to zero. We show the OSN responses to the component at different ratios in mixtures normalized to its response to the same component at $100 \%$ in a mixture (i.e. pure odor) in Fig 4.3. When two odors are blended in the same amount (50/50), and the respective responses to these two components are assumed independent from each other, the OSN response to one component in this mixture still holds $86 \%$ of the maximum response strength.

Although we can know the OSN responses to a single constituent odor depending on constituent concentration diluted in a mixture, no general rule determines the way of constituent interactions so that we cannot predict the OSN responses to a whole odor mixture. The OSN responses to odor mixtures can attribute to several 

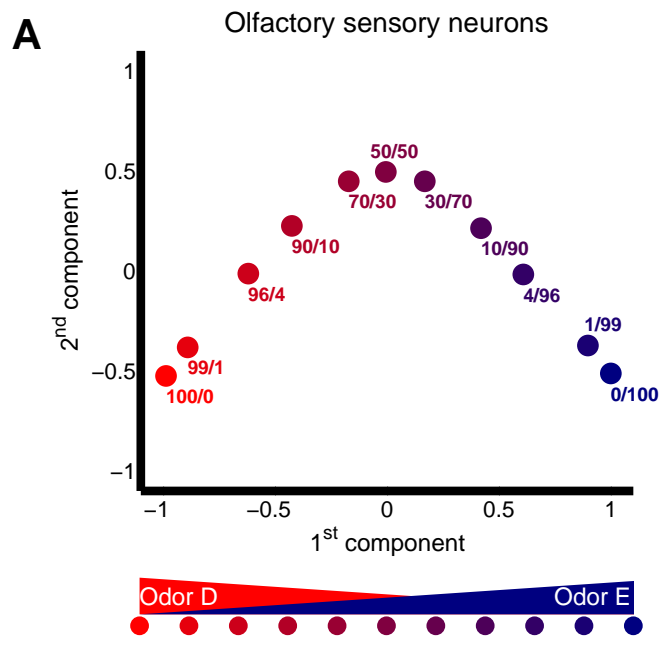

C

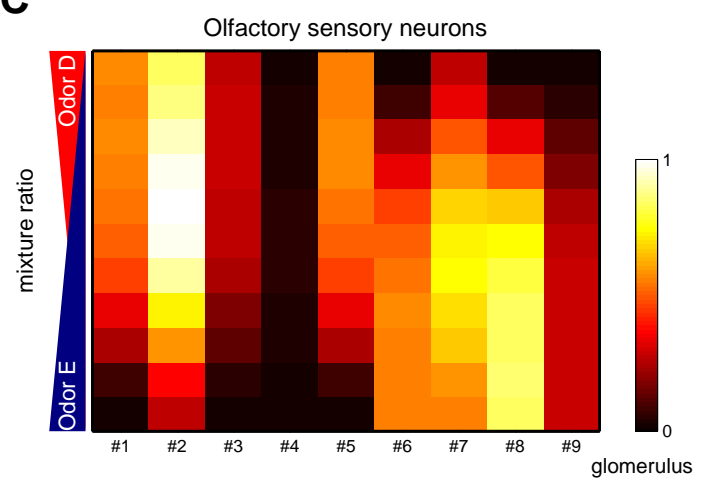

E

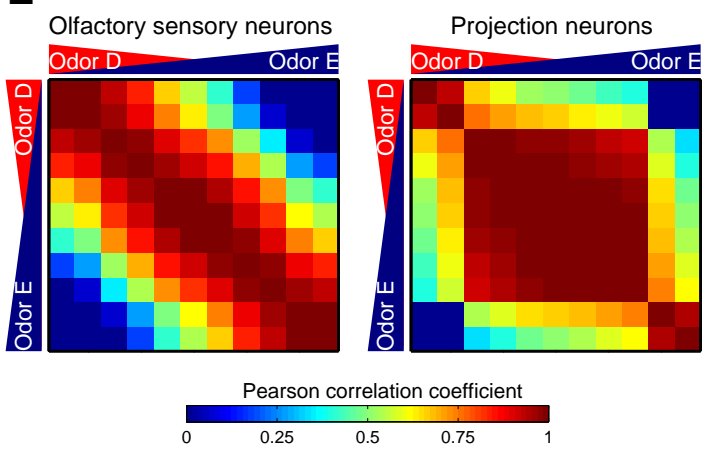

B
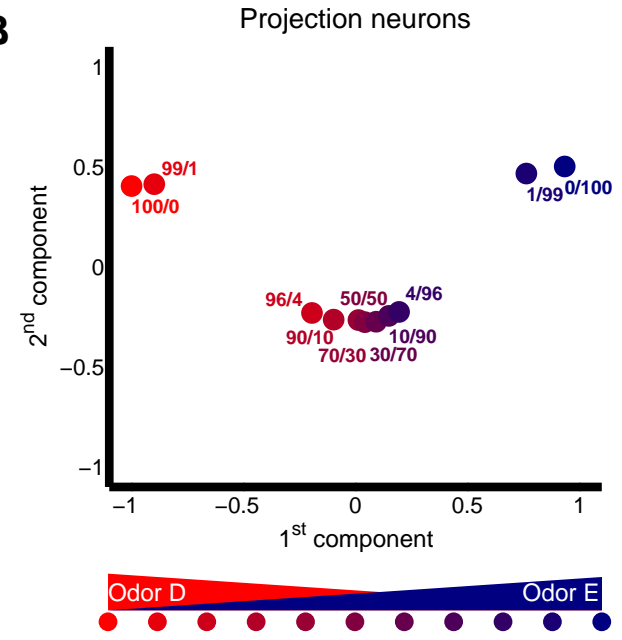

D

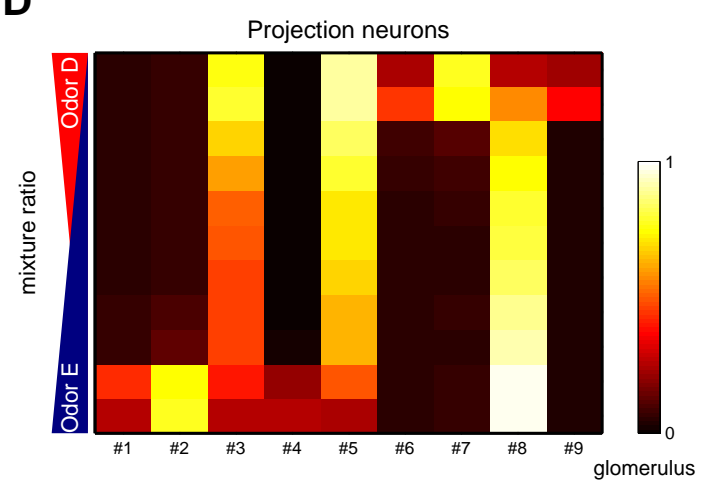

$\mathbf{F}$

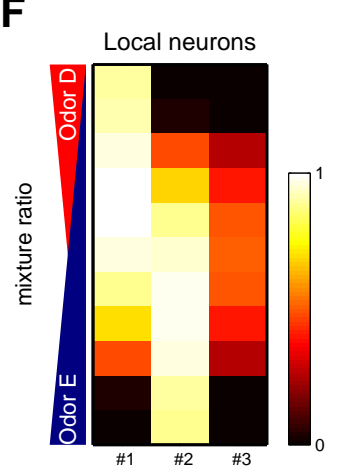


possible interactions between constituent odors including additivity, synergism, and hypoadditivity. Therefore, in the following experiments, we individually implement each of three interactions at the OSN level and investigate the effect of different interactions on processing the olfactory information of mixed stimuli in the antennal lobe.

If the response to odor mixture is simply predicted by the summation of OSN response to each component, we show the OSNs responses and PNs responses to mixtures through a series of ratios morphing from odor D to odor E in Fig. $4.4 \mathrm{C}$ and 4.4D. The results in the response matrices are the averages of neuronal responses of all neurons (OSNs or PNs) in the same glomeruli over 30 trials. We start the mixture ratios from pure odor D (100/0), and simultaneously decrease odor D and increase odor $\mathrm{E}$ in the same amount (e.g. 99/1, 96/4, 90/10, etc.) until the mixture consists of only odor E. All mixture ratios can be found in Fig. 4.4A. Although the input representations are sequentially distributed along a curve in the PCA space (Fig. 4.4A), the odor representations at the output level are generalized into three discrete clusters (Fig. 4.4B). Two clusters represent odor D and odor E, respectively, and the third cluster stands for the mixture of these two odors.

Consistent with the observations in honeybees and zebrafish $[119,137,138]$, the representations of odor mixtures are separated from those of their components. Remarkably, our theoretical work reproduces the experimental results reported by Niessing et al. (Fig 4.2), that the odor mixture, $99 \%$ odor D mixed with $1 \%$ odor E, is generalized with the pure odor D, and vice versa. The other analysis based on Pearson correlation coefficient also shows consistent results. The linear relationship between

Figure 4.4 (previous page): Separating and generalizing the odor representations in morphing between dissimilar odors when the interactions between components in OSNs are addictive. The recurrent networks categorize the odor mixtures into three discrete outputs. Lateral connection weights: $W_{L N 1}$ and $W_{L N 2}=2.4 ; W_{L N 3}=0.48$. A.B. OSN and PN representations across a series of mixture ratios in the PCA space. The OSN representations are sequentially distributed along a curve whereas the PN representations are generalized into three discrete clusters. The first two principal components explain 94.0\% variance of original PN data. C.D. Response matrices showing the responses of OSNs and PNs in each glomerulus (column) to each ratio of mixtures (row). The represented responses are the averaged responses of all neurons in the same glomeruli and normalized to the highest response in the response matrices. E. Correlation matrices representing the pairwise similarity between OSN responses and between PN responses to mixtures in different ratios. Three red blocks in the PN correlation matrix indicate the existence of three clusters at the output level. F. A response matrix showing the responses of LNs (column) to each ratio of mixtures (row). The represented responses are normalized to the highest response in the response matrix. 
different mixture ratios is represented by Pearson correlation coefficient in the form of matrices (Fig. 4.4E). By comparing the correlation matrices between the input and output levels of the antennal lobe, the recurrent networks generalize odor mixtures across different ratios from 10/90 to 90/10 and separate these intermediate mixtures from unmixed odors. The agreement of results between experimental data and our theoretical work provides further supports that our model should reflect how the olfactory system processes the odor stimuli composed of multiple qualities.

An odor stimulus containing a little heterogeneous odorant may be considered as an odor contaminated by chemical noise, and the olfactory networks can filter out this noise and represent the stimulus with its main quality. Indeed, the olfactory stimuli in natural environment are always mixtures of several quality, and such olfactory strategies give the olfactory system to focus on and to extract main odor information if the amount of other quality is little.

In addition, when the concentration fluctuation occurs in odor mixtures, the ratio between components also varies. Generalizing odors consisting of a major quality and a little amount of heterogeneous quality fluctuating in intensity can generate an invariant pattern representing the primary quality. For example, although the 99.5/0.5 and 99.9/0.1 mixtures are different odor stimuli for input, their representations can be clustered with the 100/0 mixture (i.e. single quality) at the output level by suppressing the concentration variance and accentuating the primary quality. Such generalization of multiple qualities in different ratios is similar to what we have seen in the concentration-invariant coding.

How does the recurrent networks perform the olfactory processing for separating and generalizing odor representations of mixtures in different ratios? The mechanisms are similar to what we have shown in the Chapter 3, and thus the heterogeneous connectivity of LNs may play a significant role to initiate these mechanisms. The partially-connecting LNs with different connectivity respond to different ranges of ratios. Odor D and odor E starting from $4 \%$ and above this percentage in mixtures can activate LN1 and LN2 respectively (Fig. 4.4F). The lateral connectivity of LN1 innervating glomeruli No. 1, 2, and 4 largely overlaps with the glomeruli where the OSNs are strongly evoked by odor D. So the increasing amount of odor D in mixtures can activate LN1 when the ratio exceeds the threshold between 1/99 and 4/96. On the basis of the same principles, LN2 can be evoked by odor E when the ratio is across the threshold between $99 / 1$ and $96 / 4$.

Then this feedback inhibition of LN1 results in abrupt change in PN responses between the 1/99 and 4/96 mixtures (Fig. 4.4D). The abrupt transition between PN responses in glomeruli No. 1, 2, and 4 causes the representations of odor E (0/100 and 1/99) to be far separated from the cluster standing for intermediate mixtures. Similarly, the feedback inhibition from LN2 separates the representations of odor D from those of the intermediate mixtures via glomeruli No. 6, 7, and 9. Interestingly, the simultaneous feedback inhibition from LN1 and LN2 causes PNs to be silent 

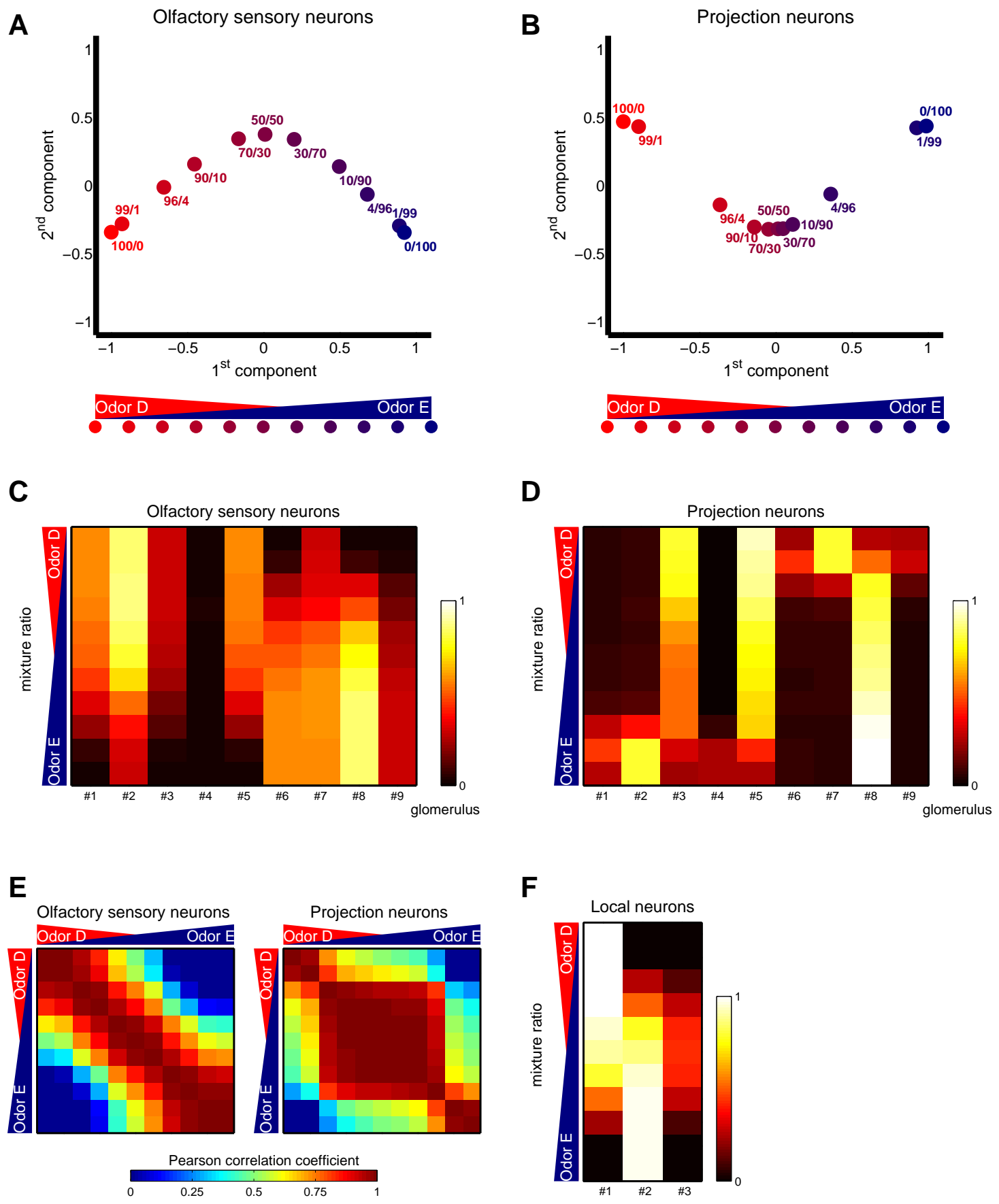

Figure 4.5: Separating and generalizing the odor representations in morphing between dissimilar odors when the interactions between components in OSNs are synergistic. Lateral connection weights: $W_{L N 1}$ and $W_{L N 2}=2.4 ; W_{L N 3}=0.48$. A.B. OSN and PN representations across a series of mixture ratios in the PCA space. The first two principal components explain $95.8 \%$ variance of original PN data. C.D. Response matrices showing the responses of OSNs and PNs, respectively. E. Correlation matrices representing the pairwise similarity between OSN responses and between PN responses to mixtures in different ratios. F. A response matrix showing the responses of LNs. Plot representations are the same as Fig. 4.4. 
and unvaried in several glomeruli across the intermediate mixtures from the ratios of $96 / 4$ to $4 / 96$, and thus generalizes the representations of these mixtures into one cluster.

Here we sum up how the representation transitions occur from one odor morphing into the other through a series of intermediate mixtures in the dynamics of recurrent networks. First, odor D only activates LN1 because of the high proximity between the connectivity of LN1 and the glomeruli where OSNs strongly evoked by odor D project their axons. This rule also explains why odor E is specifically associated with LN2. Then the representations of odor stimuli still preserve although odor D is mixed with a little amount of odor $\mathrm{E}$. When the percentage of odor $\mathrm{E}$ in mixtures increases, and the quantity of odor $\mathrm{E}$ is enough to activate the LN2, the output PN responses dramatically change because one more inhibitory LN2 is involved in the olfactory processing. Therefore, the corresponding representations of intermediate mixtures are distantly separated from the representations expressing the single odor D identity. The two simultaneously coactivated LNs suppress the PN responses in several glomeruli across a large range of different ratios and contribute to invariant output representations. The progressively increasing odor $\mathrm{E}$ in the mixtures implies the decreasing of odor D. Finally, when the percentage of odor D in mixtures decreases so that odor $\mathrm{D}$ is too little to activate LN1, the output PN responses dramatically change again. Therefore, the representations of the odor stimuli dominated by the odor E quality are separated from the representations of intermediate mixtures.

To test the effect of different interactions between constituent odors at the OSN level on this morphing experiment, we change the way of OSN responses to components in mixtures to synergism. For obtaining OSN responses to mixtures, we first set the OSN responses to components, odor $\mathrm{X}$ and odor $\mathrm{Y}$, as $R[\mathrm{X}]$ and $R[\mathrm{Y}]$ respectively. The maximum and minimum of responses to individual constituent odors are represented by $\mathbf{M A X}(R[\mathrm{X}], R[\mathrm{Y}])$ and $\mathbf{M I N}(R[\mathrm{X}], R[\mathrm{Y}])$ respectively. Then we can get the response to a mixture $R$ [mixture] by the following assumption to calculate the interactions between components in synergism:

$$
R[\text { mixture }]=\mathbf{M A X}(R[\mathrm{X}], R[\mathrm{Y}])+0.2 \times \mathbf{M I N}(R[\mathrm{X}], R[\mathrm{Y}]) .
$$

By assuming that the OSN responses are in synergistic interactions between components, we demonstrate the resulting OSN and PN responses to the odor mixtures in different ratios in Fig. 4.5C and 4.5D respectively. We find that despite the change of component interactions in OSNs, the antennal lobe can still categorize the odor mixtures into two clusters representing respective qualities and one cluster representing intermediate mixtures at the PN level (Fig. 4.5B and 4.5E). The intermediate mixtures across a large range of different ratios are also generalized into the same cluster. The LN activity of the morphing series in this experiment resembling the 

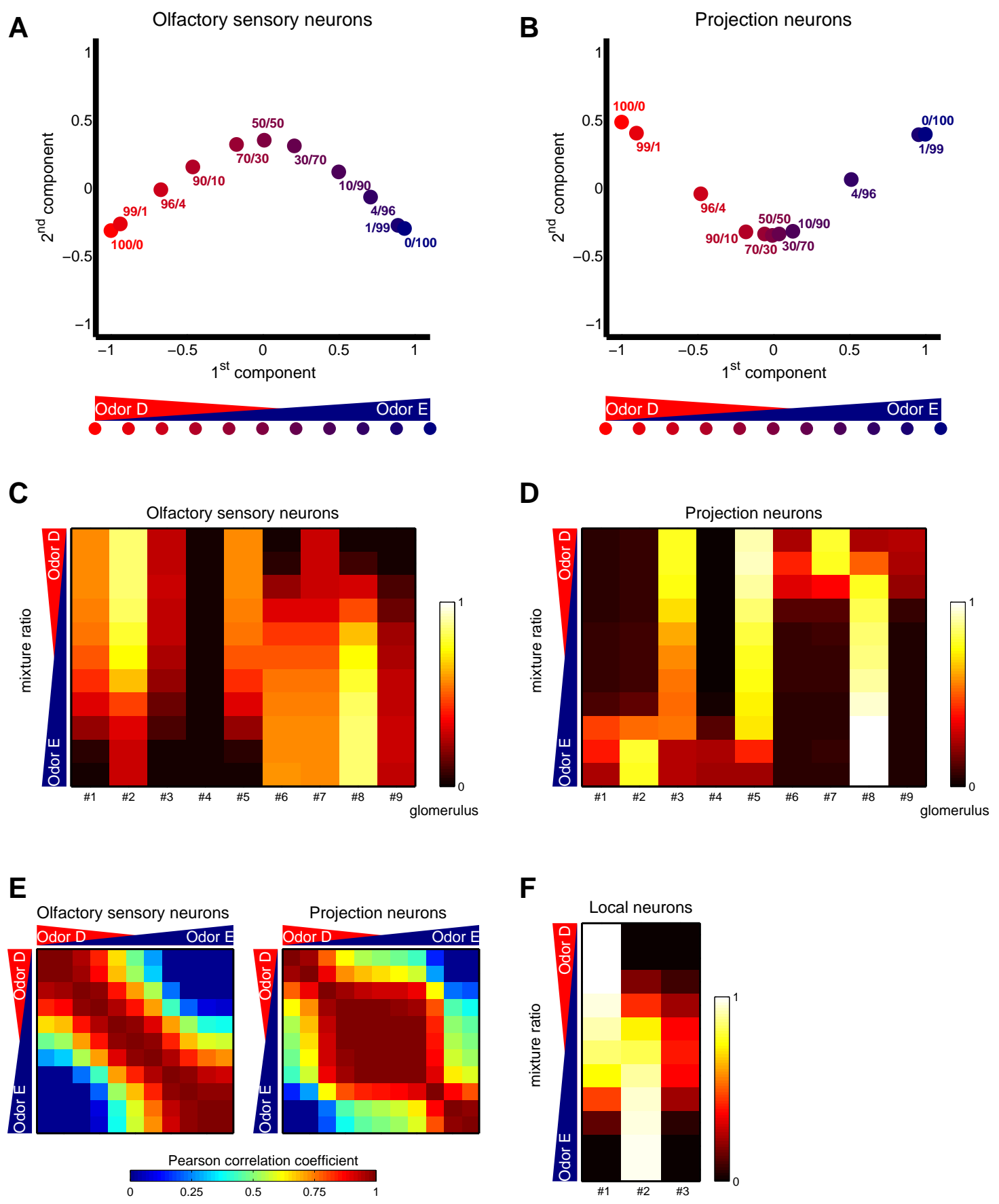

Figure 4.6: Separating and generalizing the odor representations in morphing between dissimilar odors when the interactions between components in OSNs are hypoadditive. Lateral connection weights: $W_{L N 1}$ and $W_{L N 2}=2.4 ; W_{L N 3}=0.48$. A.B. OSN and PN representations across a series of mixture ratios in the PCA space. The first two principal components explain $93.9 \%$ variance of original $\mathrm{PN}$ data. C.D. Response matrices showing the responses of OSNs and PNs, respectively. E. The correlation matrices representing the pairwise similarity between OSN responses and between PN responses to mixtures in different ratios. F. A response matrix showing the responses of LNs. Plot representations are the same as Fig. 4.4. 
A

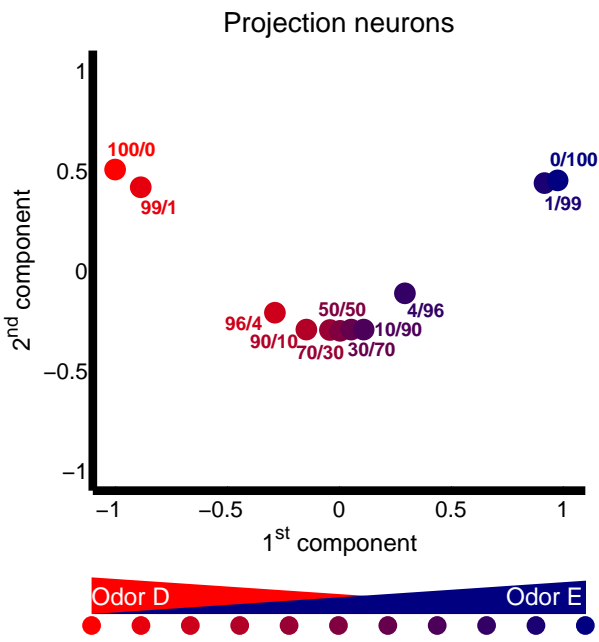

B

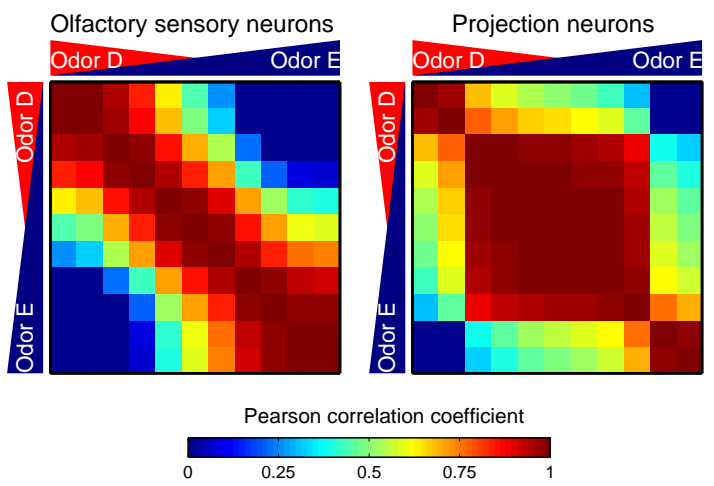

Figure 4.7: The increase of the weights of partially-connecting LNs supporting the neural circuits to generalize intermediate mixtures. Weights: $W_{L N 1}$ and $W_{L N 2}=$ 2.6; $W_{L N 3}=0.48$. A. OSN and PN representations across a series of mixture ratios in the PCA space. The first two principal components explain $96.8 \%$ variance of original PN data. B. Correlation matrices representing the pairwise similarity between OSN responses and between PN responses to mixtures in different ratios. Plot representations are the same as Fig. 4.4.

results of LNs in the earlier experiment assures the similar outcomes of olfactory processing to different types of interactions between components (comparing Fig. 4.4F with Fig. 4.5F).

We next ask how the same recurrent network responds to the hypoadditivity, another interaction way of components at the OSN level. According to the definition of hypoadditivity, the OSN response to a mixture $R$ [mixture] is equal to $\operatorname{MAX}(R[\mathrm{X}], R[\mathrm{Y}])$ given two components, odor $\mathrm{X}$ and odor $\mathrm{Y}$ in the mixture. Almost all results of all neuronal responses to mixtures in different ratios, the odor representations in the PCA space, and the linear dependence of pairs between different ratios are similar to the earlier experiments in other interaction types (Fig. 4.6). Only the representations of the 96/4 and 4/96 mixtures are not close to other intermediate mixtures within the cluster standing for odor mixtures.

The OSN responses to odor mixtures decrease in hypoadditivity compared to those in additivity and synergism so that the strength of LN inhibition also decreases (Fig. 4.6F). Indeed, as we showed in Chapter 3, the connectivity of LNs needs to be modified for getting better performance in separating or generalizing representations when different OSN response profiles are given. When we slightly raise the weights of partially-connecting LN1 and LN2 from 2.4 to 2.6, the representations of the 96/4 and 4/96 mixtures shift towards and become close to other intermediate mixtures by the strengthened inhibition (Fig. 4.7). Therefore, the antennal lobe based on our 
A

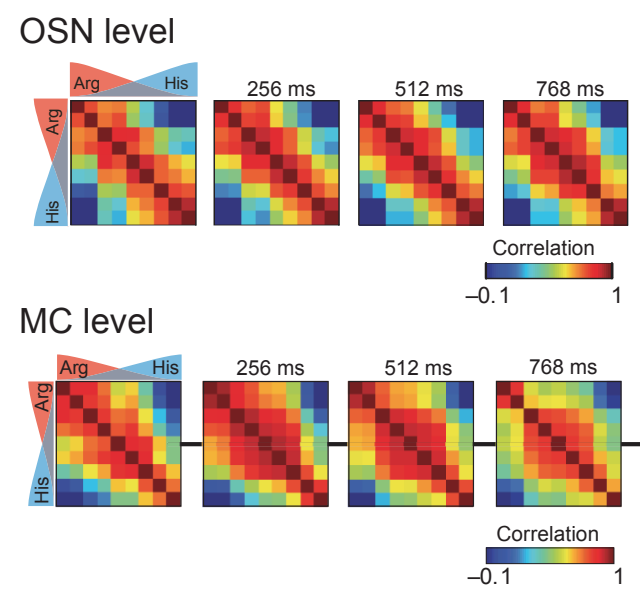

B

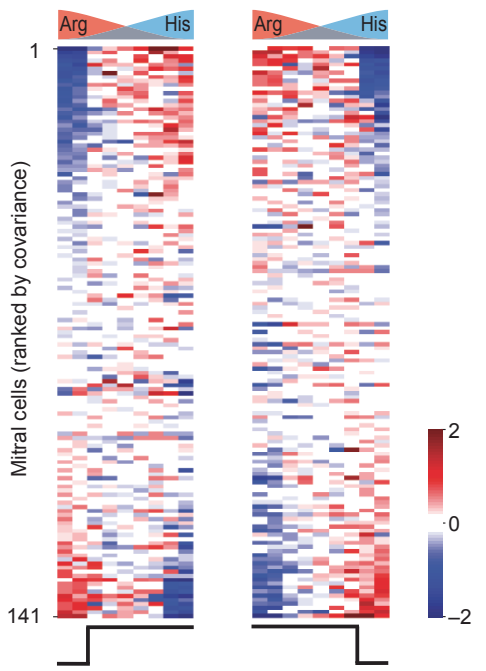

Figure 4.8: The experiments in morphing between dissimilar odors conducted by Niessing et al. to test the output pattern transition of the olfactory bulb in zebrafish. The stimuli of odor mixtures consisted of a dissimilar odor pair, Arg and His, and their ratios between two components were 100/0, 99/1, 90/10, 70/30, 50/50, 30/70, $10 / 90,1 / 99$, and $0 / 100$. The odor application lasted for more than two seconds, and the responses were measured by two-photon calcium imaging. A. Correlation matrices representing the pairwise similarity between OSN responses and MC responses to the mixtures in different ratios. The olfactory information was generalized and separated during the transformation from input OSN level to output MC level. B. Response matrices showing the responses of all $141 \mathrm{MCs}$ from seven fishes at $768 \mathrm{~ms}$ after odor onset. The response arrays were ordered by the covariance with the template below the response matrices. The MCs at the bottom of the right matrix exhibited abrupt change of responses between the 10/90 and 1/99 mixtures so that the representations of His were separated from the representations of intermediate mixtures. The same mechanisms also applied to the left matrix. Adapted by permission from Macmillan Publishers Ltd: Nature [119], copyright (2010)

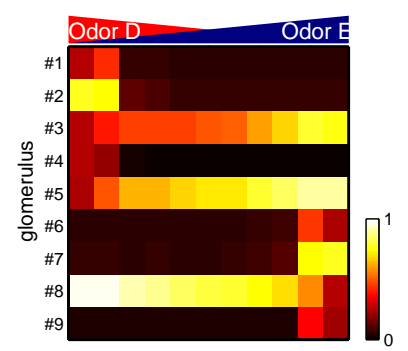

Figure 4.9: The PN responses in morphing between dissimilar odors based on our theoretical model. The response matrix is the same as Fig. 4.4D and is only counterclockwise rotated. 
proposed model can exhibit consistent results in processing this dissimilar odor pair regardless of what interactions between components occur in the site of olfactory receptors.

The dissimilar odor pair used by Niessing et al. in their experiments was two amino acid odors, Arginine (Arg) and Histidine (His) [119]. The correlation matrix from their experimental results exhibited high similarity (red area) only along the diagonal at the OSN level meaning that the mixtures are only similar to others in neighboring ratios (Fig. 4.8A). This diagonal similarity results from the distinct response patterns between dissimilar odors. In contrast to the OSN responses between mixtures, three squares filled with red and orange colors along the diagonal can be found in the correlation matrix $(768 \mathrm{~ms})$ at the output MCs of the olfactory bulb. Strong correlation between the MC response patterns to different mixtures within the squares suggests clustered representations. Remarkably, by comparing Fig. 4.7B and Fig 4.8A, our results are consistent with their findings.

Moreover, when looking more deeply and comparing our results with the data shown by Niessing et al. at the neuron level [119], we find similar neuron activity across mixture ratios. They suggested the abrupt transition between representations was caused by the sudden change of activity in a subset of MC between the 99/1 and 90/10 mixtures and the 10/90 and 1/99 mixtures shown in Fig 4.8B. Specifically, a few MCs at the bottom lines of the right matrix had strong activity at the first two columns from the right (the 1/99 and 0/100 mixtures) whereas their responses to other mixtures significantly decreased. For clear comparison, the response matrix of PNs morphing between a dissimilar odor pair based on our proposed model from Fig. 4.4D are counterclockwise rotated in Fig. 4.9. Note that the output neuron PN in the antennal lobe of insects is analogous to the output neuron MC in the olfactory bulb of vertebrates. In Fig. 4.9, the abrupt change of $\mathrm{PN}$ responses between the 4/96 and 1/99 mixtures (the third column and the second column from the right) in glomeruli No.6, 7 and 9 is similar to the behavior of MCs at the bottom lines of the right matrix in Fig 4.8B. Likewise, the distinct PN responses between the 99/1 and 96/4 mixtures causing the separated representations between odor $\mathrm{D}$ and intermediate mixtures are similar to how the MCs at the bottom lines of the left matrix separate the representations of Arg from those of intermediate mixtures (Fig 4.2).

\subsubsection{Experiment: Odor morphing between similar odors}

After we used the mixtures composed of dissimilar components as the odor stimuli, now we investigate how the neural circuits process olfactory information given mixtures made up of similar odor F and odor G. In this case, Niessing et al. reported that the odor representations are separated into two clusters at the output level when components in odor mixtures are similar (Fig. 4.10) [119]. According to the 


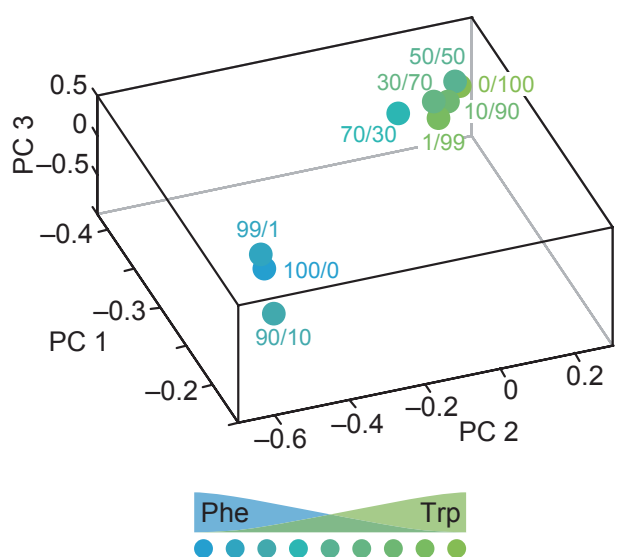

Figure 4.10: The representations in morphing between similar odors projected onto the first three principal components. The neuronal responses were measured in the MCs of zebrafish. The representations of odor mixtures were separated into two clusters, and most mixtures were generalized with one odor component (Trp). Abrupt transition occurred between 90/10 and 70/30. Figure adapted by permission from Macmillan Publishers Ltd: Nature [119], copyright (2010)

analysis of correlation matrices in their study, the relationship of odor-evoked patterns between two similar amino acid, Phenylalanine(Phe) and Tryptophan(Trp), is highly linear, and as a result, intermediate mixtures are also linearly correlated across a large range of ratios (Fig. 4.11A). Therefore, the odor-evoked OSN responses should have the following characteristics to suffice for this linear relationship among mixtures morphing between similar odors:

1. For the different types of OSNs preferring both similar odors, the response of all of them has to simultaneously increase or decrease from one odor to the other.

2. If the OSN types do not respond to one of the similar odors, they will not be activated by the other. Because these two odors are similar, the OSN types should have the same preference to both odors.

On the basis of the above assumptions, we assume that the OSN responses to two similar odors are represented in two vectors [10 4050 0] and [50 120150 0] in spike rates, respectively, and the response patterns consist of four types of OSNs. Then the correlation between these two patterns (i.e. vectors) will close to one (0.991). Now we increase the spike rate in the first element to 100 for the pattern responding to the first odor, and the response vector then becomes [100 4050 0]. Response decreasing in the first OSN types and increasing in other OSN types from the first odor to the second odor reduces the correlation between two patterns to 0.251. Next, we keep the responsive neuron in the first three elements intact, but the neuron in 

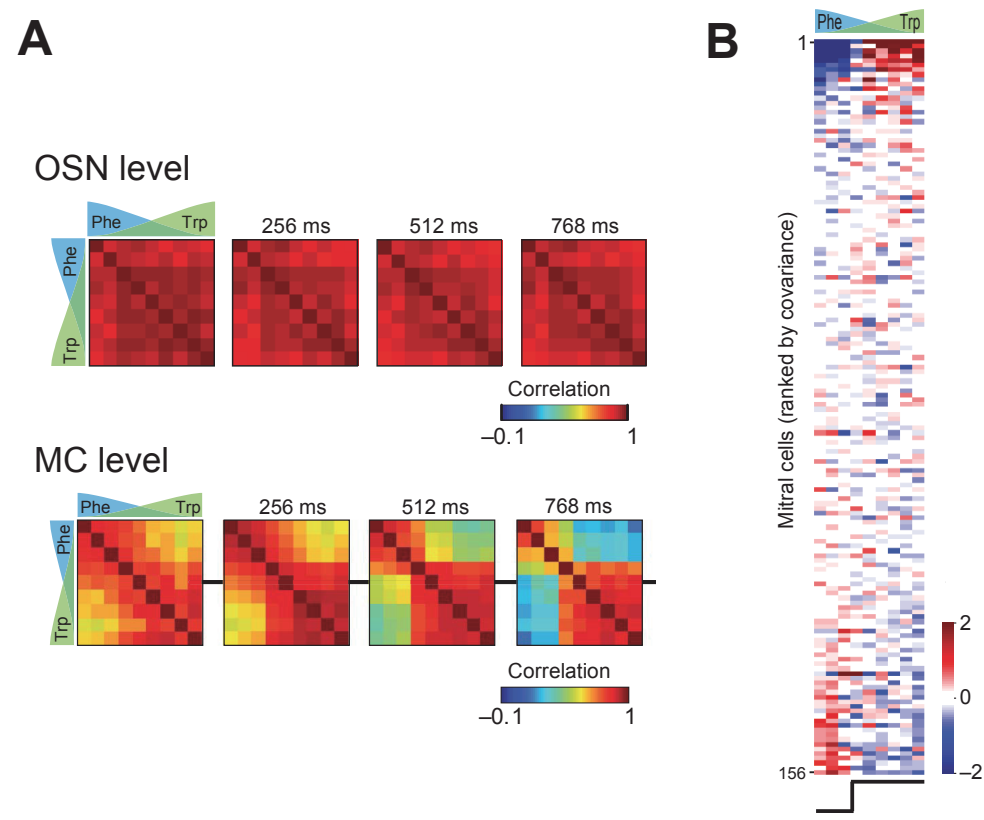

Figure 4.11: The experiments in morphing between similar odors conducted by Niessing et al. to test the output pattern transition of the olfactory bulb in zebrafish. The stimuli of odor mixtures consisted of a similar odor pair, Phe and Trp. Other details are the same as in Fig. 4.8. A. Correlation matrices representing the pairwise similarity between OSN responses and between MC responses to the mixtures in different ratios. The olfactory information was generalized and separated during the transformation from input OSN level to output MC level. B. Response matrices showing the responses of all $156 \mathrm{MCs}$ from nine fishes at $768 \mathrm{~ms}$ after odor onset. The response arrays were ordered by the covariance with the template below the response matrix. In the bottom of the response matrix, the MCs exhibited abrupt change of responses between the 99/10 and 70/30 mixtures (between the third column and the fourth column from the left) so that the representations of Phe were separated from those of other odor mixtures. Figures adapted by permission from Macmillan Publishers Ltd: Nature [119], copyright (2010)

the last element is activated by the second odor. The OSN patterns to two odors now become [10 4050 0] and [50120150100], and the correlation decreases to 0.776. Therefore, to represent the high linear relationship between two similar odors, the OSN responses have to be grounded on our two assumptions.

For the input to the recurrent networks, we use the same series of ratios in the pair of similar odors as the ratios used in the pair of dissimilar odors (Fig. 4.12A). The OSN responses to similar odor $\mathrm{F}$ and odor $\mathrm{G}$ are decided based on two assumptions discussed above, and the odor-evoked responses are listed in Table 4.2. We choose the hypoadditivity as the way of interactions between constituent odors in the site of receptors because it occurs more frequently than other interactions in OSNs [54, 
Table 4.2: OSN responses to odor $\mathrm{F}$ and $\mathrm{G}$ in each glomerulus and LN lateral connections to glomeruli.

\begin{tabular}{cccccccccc}
\hline Glomerulus No. & 1 & 2 & 3 & 4 & 5 & 6 & 7 & 8 & 9 \\
\hline \hline $\begin{array}{c}\text { OSN responses to } \\
\text { odor F }\end{array}$ & 5 & 5 & 10 & 40 & 5 & 50 & 20 & 5 & 5 \\
$\begin{array}{c}\text { OSN responses to } \\
\text { odor G }\end{array}$ & 5 & 5 & 50 & 120 & 5 & 150 & 60 & 5 & 5 \\
\hline $\begin{array}{c}\text { LN1 connection } \\
\quad\end{array}$ & $\mathrm{x}$ & $\mathrm{x}$ & & $\mathrm{x}$ & & & & & \\
$\quad\left(W_{L N 1}: 2.4\right)$ \\
$\begin{array}{c}\mathrm{LN} 2 \text { connection } \\
\quad\end{array}$
\end{tabular}

${ }^{a}$ (spikes/sec)

${ }^{b}$ The " $\mathrm{x}$ " means the connection between the LN and this glomerulus.

$136,138,140]$, and we already showed that different interactions do not influence the results of olfactory processing.

The activity of glomerular input from OSNs in the experiments of Niessing et al. did not change abruptly. Their results suggested that if an OSN did not respond to one odor, it would not respond to the other odor in the similar pair and to the mixtures consisting of both odors. The first observation is consistent with the results of OSN responses in our experiments (Fig. 4.12C). Furthermore, the second observation explicitly supports the second point in our assumptions to formulate the OSN responses to a pair of similar odors. The correlation matrix in our results indeed shows high linear correlation between mixtures in different ratios at the OSN level (Fig. 4.12E), consistent with the results of pairwise correlations reported by Niessing et al. (Fig. 4.11A).

Surprisingly, the PN activity patterns in our experiments exhibit abrupt change (Fig. 4.12D) although the OSN input strength gradually varies or keeps silent from one odor to the other. Such distinct PN responses result in different representations causing the separation of odor F from other odor mixtures and odor G (Fig. 4.12B). Similarly, in contrast to the high correlation between the odor mixtures in different ratios at input, the odor activity patterns of odor $\mathrm{F}$ are decorrelated from the patterns representing odor $\mathrm{G}$ and the intermediate mixtures at the output of the recurrent networks (Fig. 4.12E). In addition, the inhibitory LN2 responding to a series of mixtures from $90 / 10$ to $0 / 100$ attributes to the feedback inhibition onto PNs in glomeruli No. 6 and No. 7 (Fig. 4.12F). Only one LN is recruited in this morphing 
A
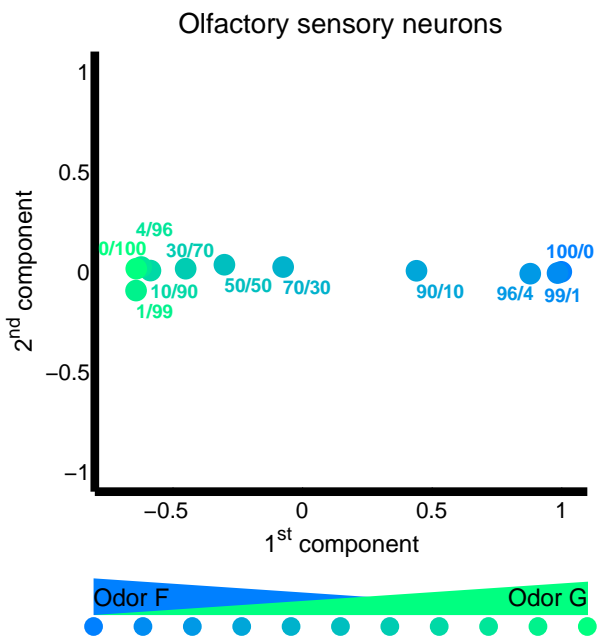

C

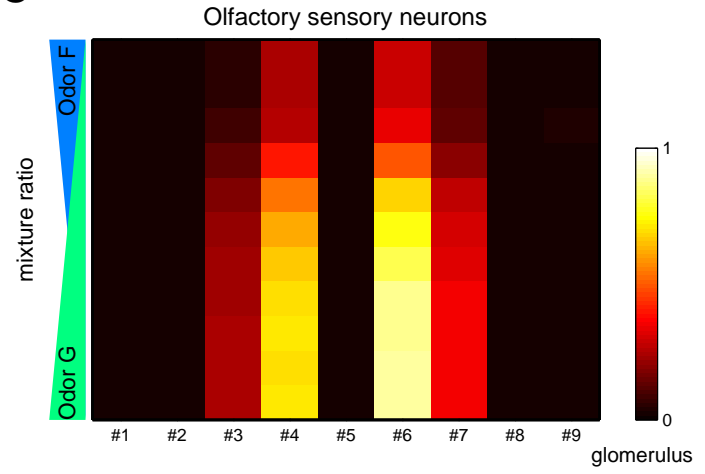

E

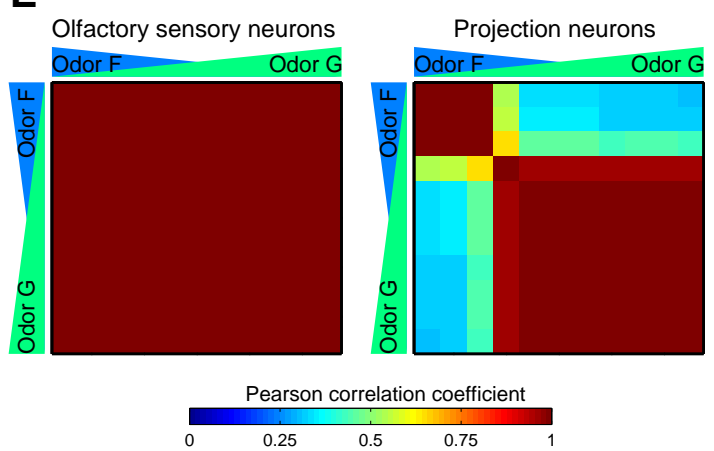

B
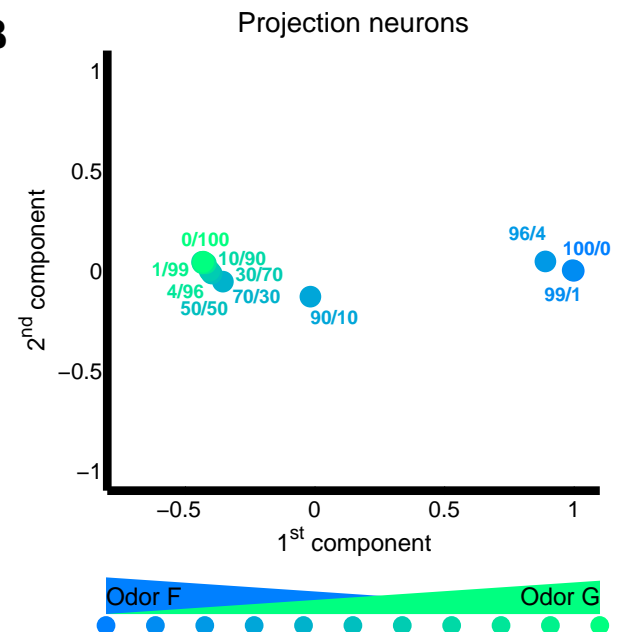

D

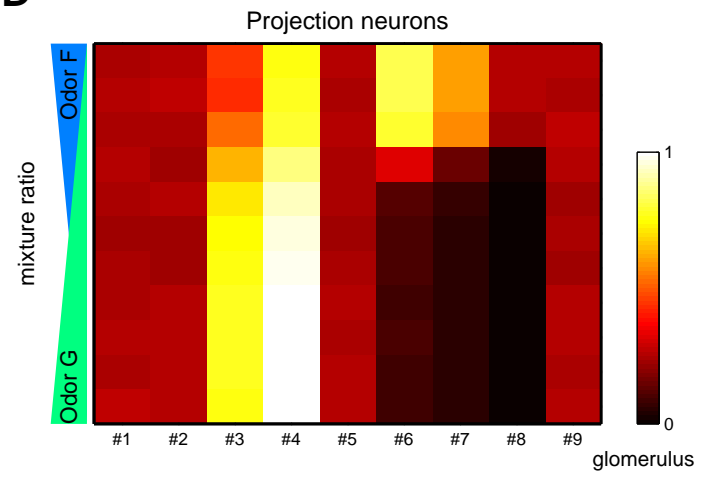

$\mathbf{F}$

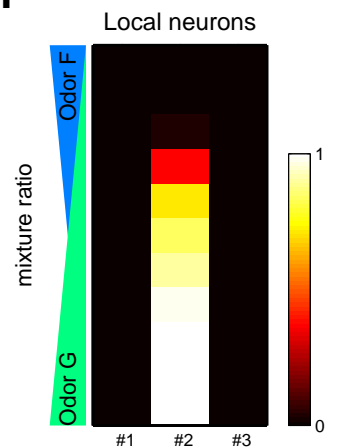

Figure 4.12: Separating and generalizing the odor representations in morphing between similar odors when the interactions between components in OSNs are hypoadditive. The recurrent networks categorize the odor mixtures into two discrete outputs. Lateral connection weights: $W_{L N 1}$ and $W_{L N 2}=2.4 ; W_{L N 3}=0.48$. A.B. OSN and PN representations across a series of mixture ratios in the PCA space. The first two principal components explain $99.5 \%$ variance of original PN data. C.D. Response matrices showing the responses of OSNs and PNs, respectively. E. The correlation matrices representing the pairwise similarity between OSN responses and between $\mathrm{PN}$ responses to mixtures in different ratios. F. A response matrix showing the responses of LNs. Plot representations are the same as Fig. 4.4. 
Table 4.3: Summary of experiments in this chapter.

\begin{tabular}{cccccc}
\hline Figure & Odor pair & $\begin{array}{c}\text { Interactions } \\
\text { in OSNs }\end{array}$ & $W_{L N 1}$ & $W_{L N 2}$ & $W_{L N 3}$ \\
\hline Fig. 4.4 & dissimilar & additivity & 2.4 & 2.4 & 0.48 \\
Fig. 4.5 & dissimilar & synergism & 2.4 & 2.4 & 0.48 \\
Fig. 4.6 & dissimilar & hypoadditivity & 2.4 & 2.4 & 0.48 \\
Fig. 4.7 & dissimilar & hypoadditivity & 2.6 & 2.6 & 0.48 \\
Fig. 4.12 & similar & hypoadditivity & 2.4 & 2.4 & 0.48 \\
\hline
\end{tabular}

experiment so that the abrupt change of $\mathrm{PN}$ patterns only occurs once when the stimuli morph from odor $\mathrm{F}$ to odor $\mathrm{G}$ through intermediate mixtures. Therefore, the output patterns of all odor mixtures are only generalized into to two clusters.

As LNs regulate which odor quantity is categorized into weak odors and strong odors, they also modulate what representations should be classified into the cluster representing a single odor quality or another cluster representing odor mixtures. According to the results of morphing experiments, one LN only can separate representations into two clusters because the categorization of representations depends on two states of LN activity, silence or activation. The LN activity determines whether inhibition is applied to output neurons via recurrent connections. The number of clusters where the output representations of odor mixtures are generalized depends on how many LNs are recruited in the odor morphing. This explains why the pairs of dissimilar odors and similar odors are generalized into different numbers of clusters.

Next, we then ask what factors decide how many LNs are utilized in the odor morphing. Each LN receives odor signals and inhibits other PNs within individual and local recurrent networks because of heterogeneous connectivity of LNs [32,56, 59-64, 106]. The activation of LN is determined by the interactions between input odor-evoked patterns and the morphology of local recurrent networks. In morphing between two dissimilar odors, two dissimilar qualities should evoke two distinct OSN response patterns so that these two patterns should then activate two different LNs, the lateral connectivity of which largely overlaps with respective OSN response patterns. Therefore, the morphing between dissimilar odors should be associated with two different LNs or two different sets of inhibition (if there are coactive LNs). In contrast, similar odor quality should elicit similar responses of OSNs in most glomeruli, and the same glomerular activation should evoke the same LN via recurrent connections. In other words, only one LN (or one set of coactive LNs) can be recruited in the olfactory processing for morphing between similar odors.

Comparing the output responses of neural circuits between our experiments and the experiments of Niessing et al., we again find resembling results in morphing between 
similar odors. The PNs in glomeruli No. 6 and No. 7 intensively respond to odor $\mathrm{F}$ (the first three rows in Fig. 4.12D), but their responses to other odor mixtures are suddenly diminished. The same case occurs in the MCs at the bottom of response matrix in Fig.4.11B. These neurons have strong responses to Phe (the first three columns from the left) whereas their responses to the rest of odor mixtures are abruptly decreased. The consistent output neuron responses in both theoretical and experimental results confirm that our model based on lateral heterogeneous connectivity and intraglomerular nonlinear transformation can fully explain the olfactory processing in separating and generalizing representations.

The experiments demonstrated in this chapter are summarized in Table 4.3 for comparing the results in different conditions.

\subsection{Discussion}

In this chapter, we have extended the study from the effect of quantity in a single odor on the outcome of olfactory information processing to how the olfactory system processes odor mixture consisting of binary components in different ratios. Our results based on PCA and correlation matrices show that the representations of each components are far separated and are decorrelated from the representations of odor mixtures. In human psychophysical experiments, subjects reported they could only recognize a new odor identity and were not able to individually identify the components of odor mixtures when they were tested in the presentation of odor mixtures made up of common and dissimilar odors $[145,146]$. The representations of odor mixtures encoded differently from those of odor components may explain why the subjects cannot easily recognize any individual components in testing odor mixtures.

We also find another olfactory strategy similar to previous concentration-invariant coding. The olfactory system may account for a small amount of odors in mixtures as irrelevant noises and encode the mixtures into the representations invariant to the concentration of minor odors. It is possible that the odor information processed in the antennal lobe or the olfactory bulb is optimized to represent odor identity (quality) instead of odor quantity. Our proposed model demonstrates how the olfactory system can suppress the concentration fluctuation either in a single odor or in the minor part of odor mixtures. For example, an odorant accounting for $0.1 \%$ or $0.01 \%$ of mixtures can be all filtered out during the olfactory processing in the first stage of the olfactory system, and the main olfactory message from the odorant dominating this odor mixture will be relayed to downstream circuits.

The separation of output representations shown in the odor morphing experiments of Niessing et al. did not arise from the activity of large numbers of MC, but from the 
abrupt change of a small subset of coordinated MC responses [119]. They reported that only $10 \%$ of MCs contributed to generate the representation separation. Indeed, on the basis of our model, we suggest that local lateral inhibition mediates the coordinated change of neural activity in one or a few specific ensembles of output neurons (MCs or PNs). These neuron ensembles switching from activated state to inhibited state morph one neural representation into another for separating odor identities or mixtures. The selective tuning of a small subset of MCs therefore underpins our model that the separation between representations is generated by local networks.

A recent study examining the same topic characterized the responses of MCs to odor mixtures in different ratios as being smooth instead of being discrete in rats [147]. A possible explanation why they have not observed similar abrupt change in their MCs may attribute to fewer numbers of MCs recorded by electrodes or their morphing analysis only based on individual MC responses. The responses of PNs in our experiments also exhibit smooth change across a large range of ratios, for example, in glomeruli No. 3, 5, and 8 (Fig. 4.4D). Considering that only a small subset of MC is involved in the odor classification, sampling a large number of output neurons should yield more complete and reliable results to reflect activity pattern change.

Another similar investigation in locusts, Shen and colleagues suggested that the output representations of odor mixtures gradually shifted in the olfactory space [144]. These findings seem in disagreement with our results that the output representations are discretely clustered, but their argument applies to only intermediate odor mixtures. Instead, both our and their studies consistently show that the representations of intermediate mixtures are converged within a subspace. Comparable results of the separation between the representations of odor mixtures and those of their components were still reported in their study. Moreover, their mixture ratios shifted from 140/0 for a component to 140/30 (i.e. 1:0.21) for the next mixtures, and the interval between their ratios is larger than the one Niessing et al. used in zebrafish: 100/0, 99/1, and 90/10 (i.e. 1:0.11) [119]. Niessing et al. reported that the generalization occurred in a single quality $(100 / 0)$ and a mixture $(99 / 1)$, and the abrupt transition between mixtures occurred in ratios from $99 / 1$ to $90 / 10$. Therefore, it is unlikely to observe these similar results of generalization and separation in locusts based on much larger intervals between mixture ratios.

Recent studies have not agreed on the prediction of the interactions between components in PNs depending on odor qualities [138, 139,142] Indeed, no general rules should exist because PN activity depends on not only the input from their presynaptic OSNs but also the lateral inhibition via recurrent networks. We cannot predict the PN response to odor mixtures merely relying on knowing OSN input without having the information of the connectivity of recurrent networks. Interestingly, several reports suggested that some PN (or MC) responses to odor mixtures were dominated by one of components and were similar to the responses to the dominating component $[140,141,144,148]$. If the interactions between components at OSNs 
are hypoadditivity, it means that the OSN activity reflects the odor information only representing one component. Therefore, the postsynaptic PNs only receive the odor information of one component from their cognate OSNs so that the PN responses to mixtures are similar to those to one component if no lateral inhibition is involved. Moreover, hypoadditivity occurs more frequently than other interactions at the OSN level, and it may explain why some PN responses to mixtures dominated by one of components were reported.

However, other PN responses to mixtures cannot be predicted based on any component responses or component quality. So back to the recurrent networks, several interactions between PNs and other neurons affect PN responses. We need to know the actual input strength from presynaptic OSNs, if the interactions are not hypoadditivity at OSNs, and the lateral inhibitory strength evoked by OSN activity patterns, if LNs connecting to these PNs are activated. In conclusion, both excitatory signals transformed by the intraglomerular function from OSNs and lateral inhibition from LNs jointly determine the PN responses to odor mixtures.

In line with our findings, another theoretical work based on glomerular random networks indicated the significance of lateral inhibition from LNs for encoding odor mixtures [149]. Our study further demonstrates how the glomerular networks respond in odor morphing through different ratios. In addition, because the connectivity of LNs is heterogeneous, we show how this specific connectivity results in different PN responses in different glomeruli such as abrupt response change in a subset of PNs. This heterogeneity also explains how different numbers of representation clusters are generated at the output of glomerular networks. Such specific relationship cannot be demonstrated by a model using random connectivity.

Our model can explain most results in the neurophysiological study of Niessing et al. [119] whereas the responses of MCs that suddenly changed their responses during the morphing between dissimilar odors were not completely silent to intermediate mixtures. It is difficult to use the model of the antennal lobe to predict the entire behavior of neuronal responses in the olfactory bulb because these two olfactory systems still slightly differ. However, both systems implement combinatorial glomerular codes to represent odors in parallel units, heterogeneous connectivity to relate specific parallel units, and interglomerular inhibition across specific parallel units to shape odor representations. These underlying mechanisms shared by both of the antennal lobe and the olfactory bulb allow us to interpret the fundamentals of how olfactory information is processed.

Although we have not referred to nonlinear intraglomerular transformation in this chapter, it does not mean that this mechanism is not important in processing odors with multiple qualities. Instead, it still plays a role in generalizing representations like in concentration-invariant coding. For example, the PN responses become saturating, and the PNs have similar responses between mixtures 1/99 and 0/100 in 
glomerulus No. 2 (Fig. 4.4D) whereas the responses of their cognate OSN differ in these two mixtures (Fig. 4.4C).

In this chapter, we have demonstrated how the neural circuits decorrelate the representations between odor mixtures and their components. Similarly, the antennal lobe or the olfactory bulb has long been thought to decorrelate different quality and increase the separation between the olfactory inputs from OSNs. Therefore, our third question concerns how the olfactory system represents a large amount of odor quality and modulates the distance between distinct qualities in the odor space. Are inhibitory LNs still responsible for separating representations between different odor qualities, or is another neural mechanism required to achieve it? We will discuss this topic in the next chapter. 



\section{Olfactory processing in modulating separation between odors}

\subsection{Overview}

In this chapter, we will investigate how neural circuits modulate representations between different odor qualities. First, we review recent studies focusing on the separability between odors during olfactory processing and then summarize that the antennal lobe or olfactory bulb is capable of separating sensory input representations from two different odor signals. Next, we analyze the experimental data from [150], and show that the same neural circuits can generalize different odor quality. The odor quality generalization is distinct from the idea commonly accepted in the olfactory processing.

We then ask why this kind of counterintuitive generalization function can emerge and coexist with the separation function in the same wiring of connectivity. Our model will explain how neural circuits with specific heterogeneous connectivity can separate representations of some odor pairs but generalize those of others.

\subsection{Increasing separation between representations}

Higher brain areas require distinct neural representations to differentiate distinct sensory stimuli. The difficulty of identifying neural representations can arise when representations are overlapping and similar. In combinatorial codes, odors activate different types of olfactory receptors, and the olfactory information is encoded in combinatorial responses of multiple OSN types [10,29, 39, 75, 112]. However, these odor-evoked patterns are not completely nonoverlapped. One OSN type can respond to many odors, and the response of OSNs are not specific to a few odors. Therefore, the odor representations at the input level are overlapping and correlated. Some studies assume that one major function of the antennal lobe or the olfactory bulb is to enhance odor discrimination and increase separation between olfactory 
inputs from OSNs $[4,5,19,101,151]$. The transformation of odor signals between the first layer and the second layer reduces overlap between representations serving as "decorrelation" in the sensory processing [151]. Such mechanisms accentuate the difference between odor representations over commonness and enable higher brain areas to extract information from nonoverlapping OSN responses.

Several experimental results confirmed that the antennal lobe took a role in increasing separation between odor representations $[53,58,138]$. In Drosophila, whereas most of odor-evoked responses at the OSN level were clustered in a subspace of the odor space, the PN responses were separated from each other and redistributed more uniformly than the OSN responses in the space [53]. Another study in Drosophila suggested that the separation between odor representations increased during the olfactory processing from the input layer to the output layer in the antennal lobe [58]. $\mathrm{PN}$ response patterns being less correlated to each other than afferent input patterns were also reported in honeybees $[57,138]$.

How can the antennal lobe attenuate overlap between odor representations and increase the separation between each other? Several theoretical works indicated that the feedback inhibition of recurrent networks could perform such functions [55, 94, 152-156]. Most of theoretical simulations implemented random feedback connections to demonstrate how lateral inhibition helped recurrent networks decorrelate input odor-evoked patterns. One of these works suggested that heterogeneous recurrent networks leaded to better separation between representations than global feedback networks when the response frequency of each OSN type was unequal [155]. In this work, the inhibition strength onto the $\mathrm{PNs}$ in a glomerulus was related to the similarities of OSN responses between this glomerulus and other glomeruli. In other words, the inhibition strength needed to be tuned based on the OSN response patterns. Interestingly, the frequency of one OSN type responding to odors is indeed different from those of other types [39]. Some OSN types are evoked by many odors whereas other OSN types respond to much fewer odors.

Another study suggested that the local and global feedforward inhibition performed different functions in recurrent networks, and the former, not the latter, resulted in decorrelation [152]. In addition, distinct LNs in local recurrent networks could reduce different parts of overlap between a pair of odors by inhibiting output neurons in confined and different sets of glomeruli [157]. The different inhibitory feedback could contribute to various degrees of separation between representations, and the neural circuits could get better resulting separation by selecting the activation of specific LNs. All these studies make it clear that heterogeneous recurrent networks play a significant role in separating representations and increasing the discrimination between odors. In the next section, we will use these ideas to interpret the findings in neurophysiological experiments. 


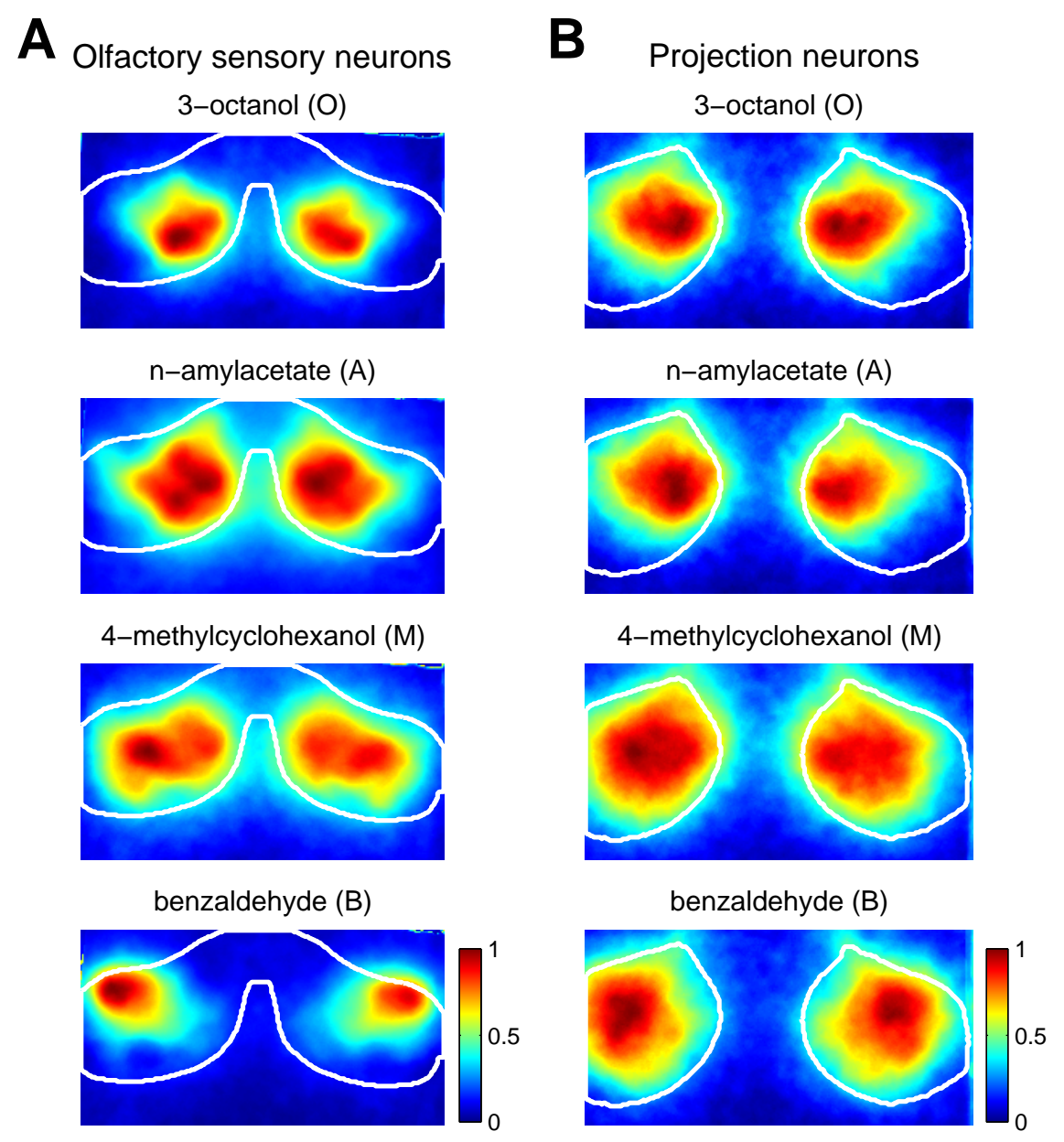

Figure 5.1: Calcium activity in the antennal lobes evoked by four different odors 3octanol (O), n-amylacetate (A), 4-methylcyclohexanol (M), and benzaldehyde (B), respectively. A. The calcium imaging representing the neuronal activity at the OSN layer. Images are averaged over eight individual flies and 3-5 trials. Each averaged image is normalized to the maximum value of respective images. The signal strength is indicated by a color bar. White contours define the outline of the antennal lobes. B. The calcium imaging representing the neural activity at the PN layer. Image representations are the same as (A.). Note that the neuronal activity in OSNs evoked by 3 -octanol and $n$-amylacetate is distinct whereas the neuronal activity in $\mathrm{PNs}$ responding to 3 -octanol and $n$-amylacetate is similar.

\subsection{Imaging data analysis}

The calcium concentration correlates with neuronal activity [158-160], and measuring the calcium intensity reflects the dynamics in large numbers of neurons simultaneously. Recent advances in the use of two-photon microscopy coupling with genetically encoded calcium-sensitive fluorescence protein can allow us to visualize 
calcium concentration change $[9,10,161]$. If a neuron type has a known genetic identity, the large-scale neural activity of that particular type can be recorded by imaging the neural circuits of live animals bearing transgenes based on that genetic identity. For example, an odorant receptor gene, Or83b, is broadly expressed in OSNs in Drosophila [25, 30, 162], and experimentalist can monitor the odor-evoked responses of OSNs in Drosophila bearing the Or83b-Gal4 and the UAS-GCaMP transgenes by two-photon microscopy $[10,54,107,150]$.

In a set of two-photon imaging data recording the neuronal responses at the OSN and PN levels in Drosophila [150], we find counterintuitive results of olfactory processing in the antennal lobe. Four different odors, 3-octanol (O), n-amylacetate (A), 4methylcyclohexanol (M), and benzaldehyde (B), were used to stimulate animals in the imaging experiments, and we show the resulting calcium activity of input neurons and output neurons in the antennal lobes in Fig. 5.1. Surprisingly, the experimental data indicate that the neuronal signals of the pair, $n$-amylacetate and 3-octanol, are similar in the PN ensembles although the odorant-evoked patterns of this pair in the OSN ensembles are distinct. The activity patterns at OSNs and at PNs evoked by the other two odors, 4-methylcyclohexanol and benzaldehyde, are different from those evoked by $n$-amylacetate or 3-octanol. Therefore, some odors are still separated during the odor transformation from OSNs to PNs whereas one particular pair $(\mathrm{O}$ and $\mathrm{A})$ is generalized.

For further analyzing the patterns of odor-evoked calcium activity, we use thresholding to define the regions of interest (ROI) in the calcium background images of antennal lobes. The pixel intensity of background images is averaged across 8 individual flies and normalized between 0 and 1. After that, we choose the pixels of which the normalized intensity is greater than 0.40 and 0.65 as the ROIs of calcium images for OSNs and PNs respectively (white circumference lines in Fig. 5.1). The threshold selection depends on the contours of ROIs capable of reflecting the anatomical location of neurons of interest.

We use PCA to reduce high-dimensional calcium imaging data to the first three dimensions that account for most of the variance. The principle components are ranked according to their contribution to the total variance of original data. Only the calcium signals within the ROIs are analyzed in PCA. Here, the calcium signals in the ROIs (7575 data points for the OSN imaging and 5890 data points for the PN imaging) are reduced to the three principle components that turn out to keep nearly $90 \%$ of the variability of the original signals.

The neuronal responses to four odors represented by calcium images are projected onto the space spanned by the first two principal components (Fig. 5.2A and 5.2B). The pair of $\mathrm{O}$ and $\mathrm{A}$ is generalized at output $\mathrm{PNs}$ (Fig. 5.2B) whereas these two odors are separated at input OSNs. Other all possible pairs out of four odors are consistently separated during the olfactory processing in the antennal lobe. To reduce inter-individual variability between flies, we calculate the mean responses over 

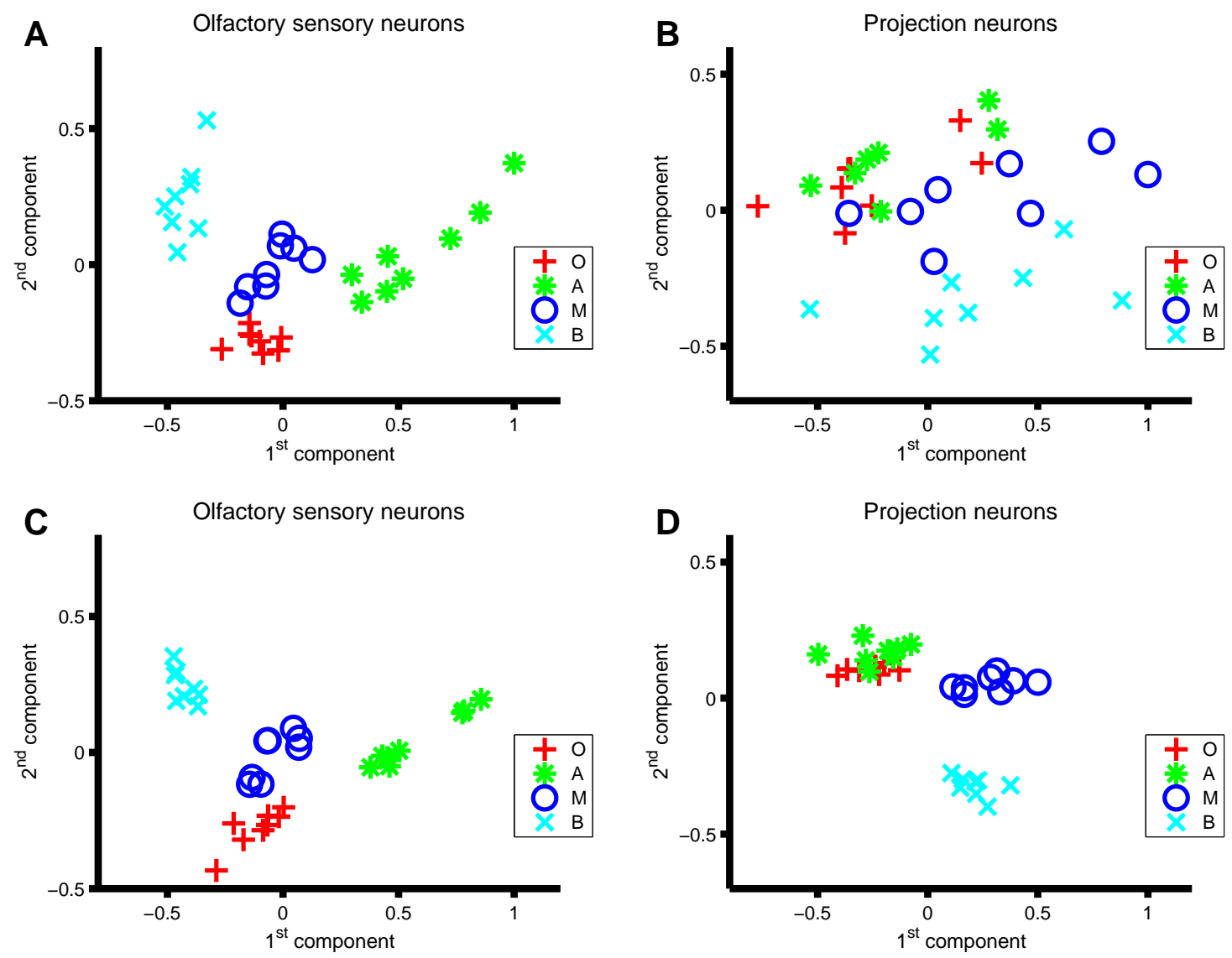

Figure 5.2: Odor-evoked calcium activity across four odors measured in OSNs and PNs projected onto the space spanned by the first two principal components. A. Odor-evoked calcium activity in OSNs represented by the first two principle components by using dimensional reduction PCA. Different markers indicate different odors as indicated in the legend. Each single marker represents a measurement of an individual animal (eight flies). B. Odor-evoked calcium activity in PNs represented by the first two principle components by using dimensional reduction PCA. The first two components explain $81.5 \%$ variance of original calcium signals. C.D. As in (A.)(B.), but inter-individual variability is removed by subtracting the mean responses over four odors in each animal. The variance of responses to the same odor becomes less after reducing inter-individual variability, suggesting that most of response variability attributes to inter-individual variability. Note that in PNs, but not in OSNs, the activity patterns evoked by $\mathrm{O}$ and $\mathrm{A}$ are generalized.

four odors in each animal and subtract each mean response from the responses to odors in corresponding animals. After the mean removal, the dimension of imaging signals is again reduced by PCA and represented in the first two principal components of PCA space (Fig. 5.2C and 5.2D). The variance of responses to the same odor becomes less after reducing inter-individual variability, suggesting that most of 


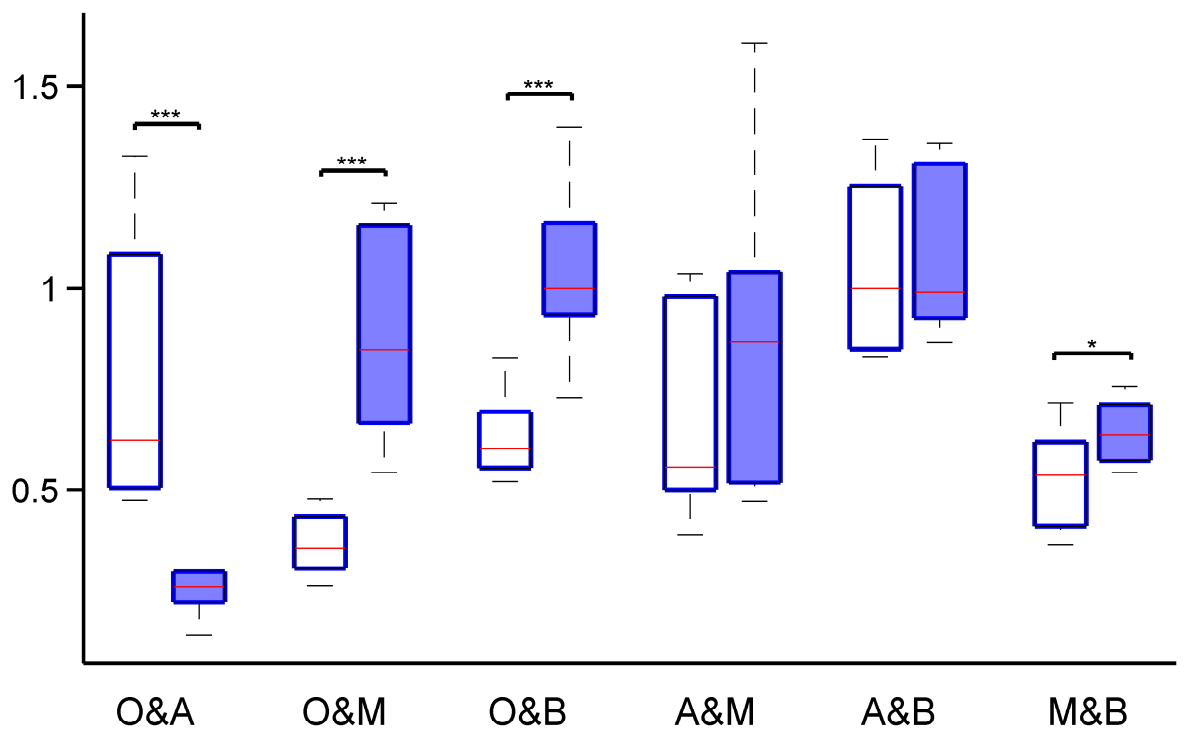

Figure 5.3: Relative Euclidean distances between the calcium activity patterns of each odor pair. The distances based on the first three principal components for each odor pair are combined across flies and are normalized to the highest median distances among the odor pairs in OSNs and in PNs, respectively. The first three components explain $89.6 \%$ variance of original calcium signals. The boxes from pairs in PNs are shaded. For each box, the central red mark is the median, and the edges of the box are the first and third quartiles. The ends of the whiskers are calculated by the mean plus or minus 2.7 variance. Relative distances that significantly differ between OSNs and PNs in one odor pair are represented with asterisks (*: $\mathrm{p}<0.05$; ***: $\mathrm{P}<0.0001$; all pairwise comparisons are established by Wilcoxon rank-sum tests). Note that the relative Euclidean distances distributed in a narrow range (e.g. O\&A in $\mathrm{PNs}$ ) mean that some response variability to an odor in Fig. 5.2 results from inter-individual variability.

response variability attributes to inter-individual variability. When inter-individual variability is reduced, it is clear that the representations between $\mathrm{O}$ and $\mathrm{A}$ are indeed clustered, but they are separated from the representations of $\mathrm{M}$ and $\mathrm{B}$ at the PN level (Fig. 5.2D).

Next, we calculate Euclidean distances between the calcium activity patterns of each odor pair based on the first three principal components. All distances are combined across flies in each odor pair and are normalized to the highest median distances among the odor pairs in OSNs and in PNs, respectively. The relative distances are shown as a box plot in Fig. 5.3, and the boxes from pairs in PNs are shaded. The relative distances of some pairs are significantly larger at the PN level than at the OSN level (M\&B: $p<0.05$; O\&M and O\&B: $P<0.0001$; Wilcoxon rank-sum tests, $n=8)$. The increasing distances between OSN and PN levels suggest that the neural circuits in the antennal lobe separate the representations further between these odor 
pairs. Consistent with the results of calcium singles projected onto the PCA space, the relative distances of the pair O\&A significantly decrease $(P<0.0001$; Wilcoxon rank-sum tests, $n=8$ ). The relative Euclidean distances distributed in a narrow range (e.g. O\&A in $\mathrm{PNs}$ ) mean that some response variability to the same odor in Fig. 5.2A and 5.2B results from inter-individual variability.

The generalization of odor representations is rarely reported, but the consistent results in behavioral experiments support that generalization occurring at the output neurons is not an artifact of calcium signals [150]. Our analysis of the experimental data suggests that the antennal lobe can both increase and decrease separability in the same neural circuits. If the heterogeneous inhibitory feedback performs olfactory computation and decorrelates odor-evoked patterns, could it also generalizes distinct input patterns? Next, we will interpret how the heterogeneous connectivity modulates separation in the antennal lobe based on our model.

\subsection{Results in theoretical investigations}

\subsubsection{Effects of recurrent connectivity on separability}

The feedback inhibition via different recurrent connectivity may modulate separation between odor pairs. If the overlap between inputs is attenuated by lateral inhibition, the outputs in the PN layer will become more separated. These are known facts, and the studies we referred in the section 5.2 only focus on this aspect. However, it is possible that inhibiting the differences of odor-evoked patterns and keeping their commonness intact will generalize these representations.

We first demonstrate how the recurrent networks based on our model increase separation between odors in the antennal lobe. All model parameters are kept the same as earlier experiments. The recurrent networks in this experiment consist of 50 glomeruli, and the numbers of OSNs and PNs are still 30 and 3 per glomeruli respectively. The responses of OSN types to two odors along glomeruli are described by Gaussian distribution centered in glomeruli No. 18 and No. 32 (solid blue and green lines in Fig. 5.4A). The spike rates in each glomerulus are the averages of neuron responses in individual glomeruli across 30 trials and are then normalized to the maximum spike rate.

Because the overlap of two input patterns is between the centers of the two bell curves, local recurrent networks specifically inhibiting the overlap, not the difference (the OSN responses in glomeruli No. 1 to 18, and No. 32 to 50), should decorrelate these patterns. We evaluate this assumption by giving higher strength of inhibitory feedback connections to the glomeruli where two input patterns overlap. 

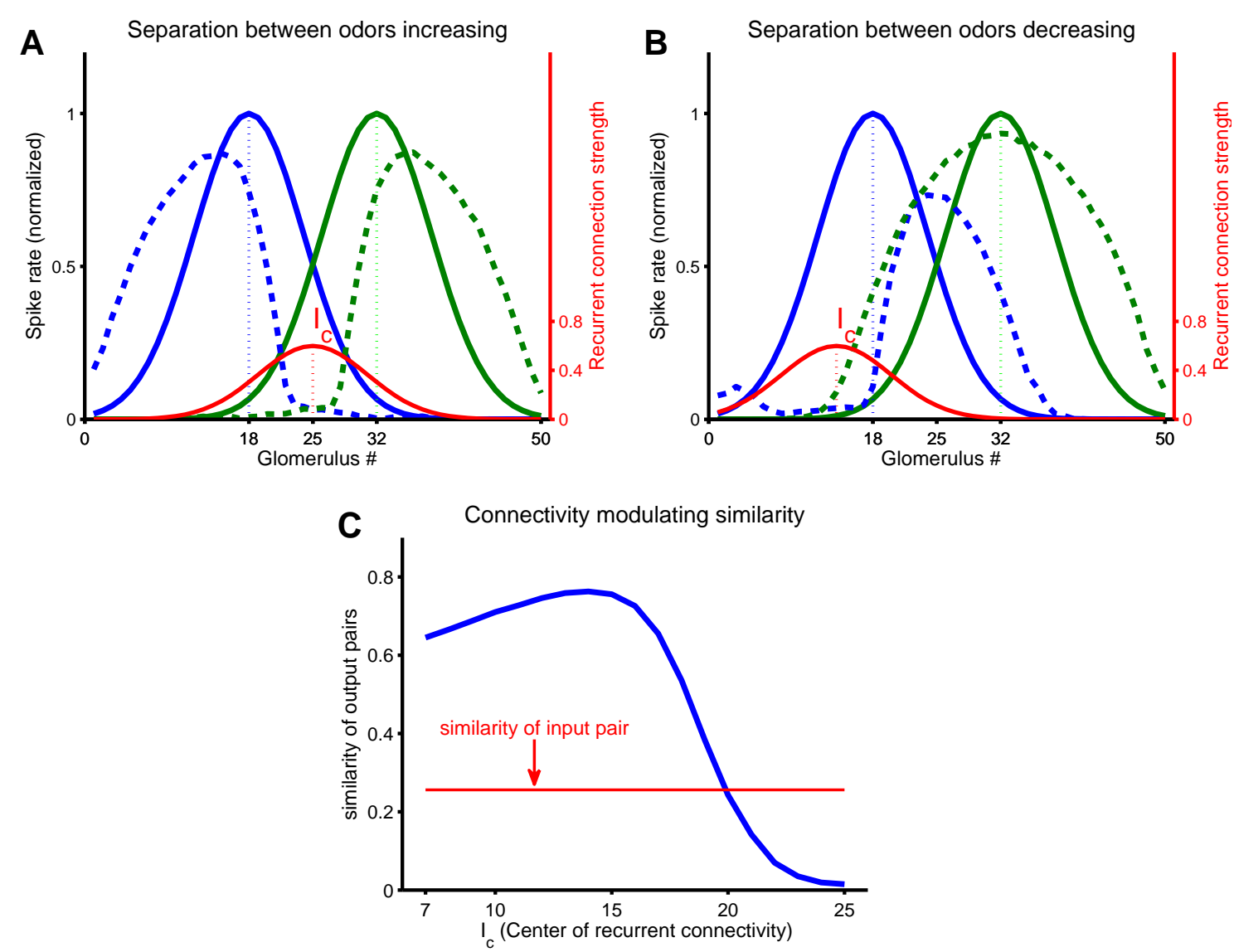

Figure 5.4: Effects of recurrent connectivity on separability. The responses of OSN types to two odors along glomeruli are described by Gaussian distribution centered in glomeruli No. 18 and No.32, plotted in solid blue and green lines respectively. All spike rates are normalized to the maximum spike rate. The weights of recurrent connections are also characterized by Gaussian distribution and plotted in solid red line. The weight at the center equals 0.6. All Gaussian curves have the same variance 6 . The dash lines represent the PN responses, and the curves in blue and green indicate the neuronal activity evoked by corresponding odors. A. The local recurrent networks specifically inhibit the overlap between input patterns (i.e. in glomeruli No. 20 to 30), the response patterns in PNs are more separated than in OSNs. B. The local recurrent networks specifically inhibit the differences between input patterns (i.e. in glomeruli No. 1 to 15), the response patterns in PNs are less separated than in OSNs. C. The similarity between outputs as the function of the center of recurrent connectivity. The similarity between the input pair is 0.26. Shifting the center of recurrent connectivity with the same shape as in (A.) and (B.) can modulate the similarity (or separation) between odor pairs at output. 
The weights of recurrent connections are characterized by Gaussian distribution centered in glomeruli No. 25, and the weight at the center equals 0.6 (solid red line in Fig. 5.4A). In the recurrent networks, one LN and all glomeruli are linked via feedforward and feedback connections, and the weights of reciprocal connections are the same for each direction. As a result of inhibiting the overlap, the response patterns in PNs (dash lines in Fig. 5.4A) are more separated than input patterns. Because of nonlinear intraglomerular transformation, a few responses at OSNs can be boosted at postsynaptic PNs (e.g. in glomeruli No. 3 or No. 47).

For the same pair of input patterns, different recurrent connectivity could perform another function in the antennal lobe. Local recurrent networks specifically inhibiting the differences between input patterns should cause them similar. Now, we keep the shape of inhibition strength the same and shift the center of the bell curve to glomeruli No. 14 (solid red line in Fig. 5.4B). The OSN responses in glomeruli No. 1 to 15 to one odor (solid blue curve in Fig. 5.4B) are stronger than those to the other odor (solid green curve in Fig. 5.4B). The LN specifically inhibiting the output neurons in glomeruli from No. 1 to 15 can attenuate the differences between odors at the output, and the resulting responses are depicted by the two dash lines in Fig. 5.4B. Interestingly, such recurrent connectivity increases the overlap between odors, and the PN responses to two odors in glomeruli No. 19 to 23 are similar whereas the responses of their cognate OSNs are different. Therefore, a pair of distinct odors can be generalized at the output of neural circuits by recurrent inhibition. This may explain why two odor qualities in calcium imaging experiments are clustered at PNs.

To demonstrate how different connectivity modulates the separation between two odors, we represent the similarity between two response patterns $R_{M}$ and $R_{N}$ along glomeruli as:

$$
S_{M, N}=\frac{\left\langle R_{M}, R_{N}\right\rangle}{\left\|R_{M}\right\|\left\|R_{N}\right\|}
$$

where $\left\langle R_{M}, R_{N}\right\rangle$ is the inner product of $R_{M}$ and $R_{N}$, and $\left\|R_{M}\right\|$ and $\left\|R_{N}\right\|$ are the norms of two vectors $R_{M}$ and $R_{N}$ respectively [87,94]. When two odors are decorrelated in the recurrent networks, the similarity between odors will decrease. The similarity between OSN response patterns in this experiment is 0.26. If the similarity between outputs is lower than this value, it means that the overlap between representations is reduced during the olfactory processing. Here, we retain the inhibition strength in the same bell shape and only shift its center along glomeruli to see the effect of different connectivity on modulating the separation between two inputs. We represent the relationship between the similarity of an output pair and the center of recurrent connectivity in Fig. 5.4C. When the center of recurrent connectivity is located at glomeruli from No. 21 to 25, the lateral inhibition attenuates the overlap between two inputs and then separates them. Therefore, these resulting similarities of output pairs are lower than that of the input pair. In contrast, 
Table 5.1: OSN responses to four odors in each glomerulus and LN lateral connections to glomeruli.

\begin{tabular}{|c|c|c|c|c|c|c|c|c|c|}
\hline Glomerulus No. & 1 & 2 & 3 & 4 & 5 & 6 & 7 & 8 & 9 \\
\hline $\begin{array}{c}\text { OSN responses } \\
\text { to } \mathrm{O}^{a}\end{array}$ & +++ & $\bullet$ & + & $\bullet$ & $\bullet$ & $\bullet$ & ++ & ++ & + \\
\hline $\begin{array}{c}\text { OSN responses } \\
\text { to } \mathrm{A}^{a}\end{array}$ & ++ & $\bullet$ & $\bullet$ & $\bullet$ & + & +++ & $\bullet$ & ++ & + \\
\hline $\begin{array}{l}\text { OSN responses } \\
\text { to } \mathrm{M}^{a}\end{array}$ & $\bullet$ & ++ & $\bullet$ & $\bullet$ & + & ++ & $\bullet$ & + & +++ \\
\hline $\begin{array}{c}\text { OSN responses } \\
\text { to } \mathrm{B}^{a} \\
\end{array}$ & $\bullet$ & +++ & $\bullet$ & ++ & + & ++ & $\bullet$ & + & • \\
\hline $\begin{array}{l}\text { LN1 connection } \\
\\
\quad\left(W_{L N 1}: 2.0\right)\end{array}$ & $\mathrm{x}$ & & & & & $\mathrm{x}$ & $\mathrm{x}$ & & \\
\hline $\begin{array}{l}\text { LN2 connection } \\
\\
\quad\left(W_{L N 2}: 2.0\right)\end{array}$ & & & $\mathrm{x}$ & & $\mathrm{x}$ & & & & $\mathrm{x}$ \\
\hline $\begin{array}{l}\text { LN3 connection }{ }^{b} \\
\quad\left(W_{L N 3}: 0.6\right)\end{array}$ & $\mathrm{x}$ & $\mathrm{x}$ & $\mathrm{x}$ & $\mathrm{x}$ & $\mathrm{x}$ & $\mathrm{x}$ & $\mathrm{x}$ & $\mathrm{x}$ & $\mathrm{x}$ \\
\hline
\end{tabular}

the lateral inhibition acting on the differences between two inputs will increase the similarity between output pairs.

The modulation of separation between odors depends on the recurrent connectivity of inhibitory LNs. In these experiments, the pair of input patterns is the same, but the output results can be either more separated or generalized. Different results attribute to different connectivity. Based on the same idea, we will further explain how the same wiring of neural circuits in the antennal lobe can simultaneously increase and decrease separation between different pairs of odors.

\subsubsection{Increasing and decreasing separation in the same wiring of lateral connectivity}

We again implement our model to investigate how the same wiring of recurrent connectivity can decrease separation between some pairs of odors and increase separation between others simultaneously. We assume four odors evoking distinct response 

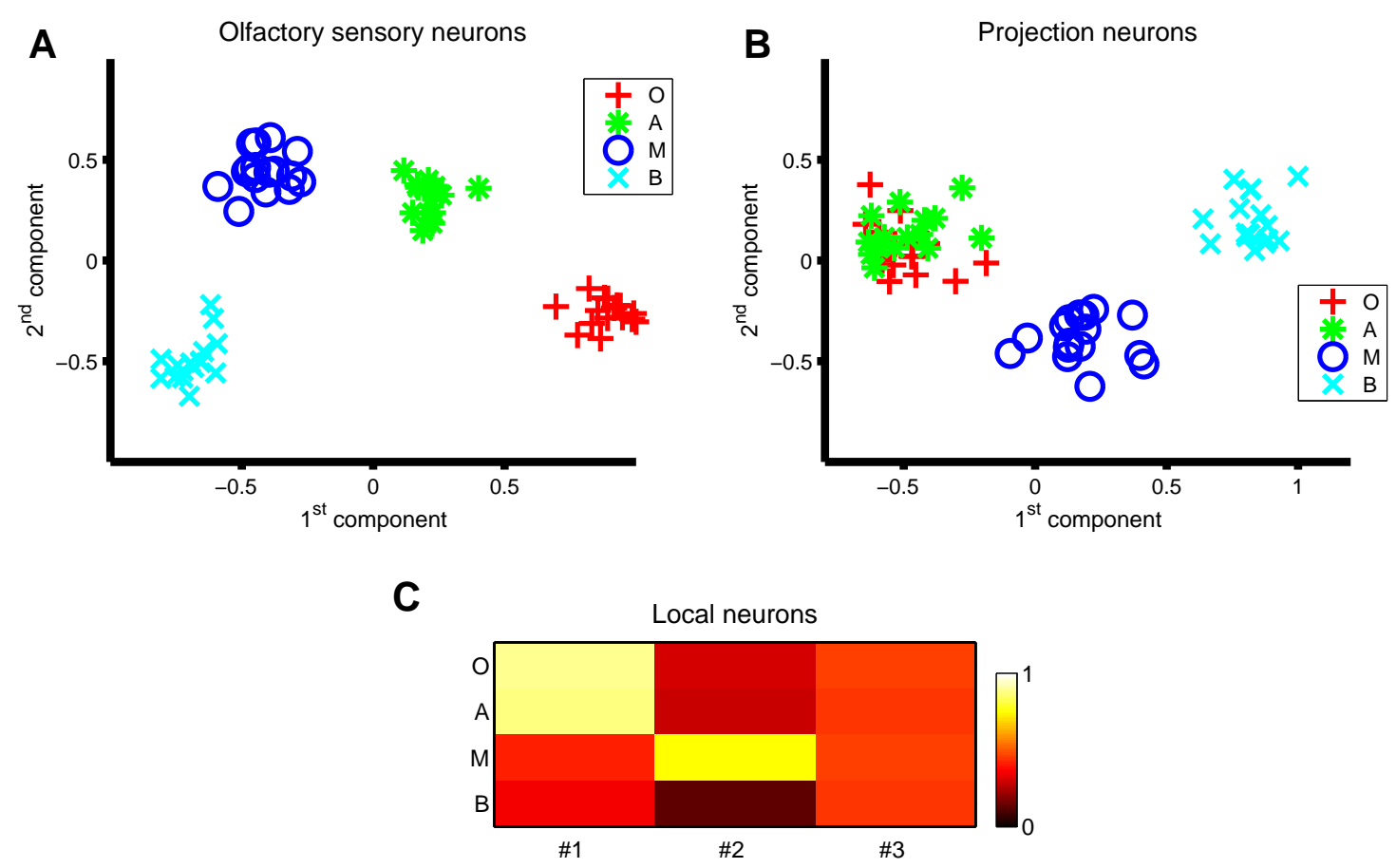

Figure 5.5: Modulating the separation between odors in the same wiring of lateral connectivity. A. The OSN responses to four odors based on theoretical simulation results. Each point represents a measurement of one trial. To simulate the noise generated in experimental data, we add Gaussian noise in the spike trains of OSNs. B. The PN responses to four odors based on theoretical simulation results. The first two principal components explain $90.26 \%$ variance of original data. Two odors $\mathrm{O}$ and $\mathrm{A}$ in $\mathrm{PN}$ ensembles are generalized although their odorant-evoked patterns in (A.) are separated. Other odor pairs are separated both in OSNs and PNs. The separation between odor representations can be either decreased or increased in the same wiring of recurrent connectivity. C. A matrix showing the responses of LNs (column) to each odor (row). The responses are normalized to the highest response to all odors in all LNs.

patterns in OSNs (Table 5.1), and thus odors are separated at the input level. Note that $\mathrm{A}$ and odor $\mathrm{A}$ used in chapter 3 stand for different meanings. Here, the term "A" is used to indicate $n$-amylacetate, and we want to reproduce the results that a specific pair, $\mathrm{O}$ and $\mathrm{A}$, are generalized at output whereas they are separated at the input of the antennal lobe. Odor $\mathrm{A}$ is another odor we used in chapter 3 to show how neural circuits encode odor quantity.

The recurrent networks consist of two partially-connecting LNs (LN1 and LN2) and one fully-connecting LN (LN3). We set specific connectivity of inhibitory LN1 to be capable of attenuating the differences between OSN responses patterns evoked by $\mathrm{O}$ and $\mathrm{A}$. To achieve this purpose, we inspect the input patterns and find the 
OSN types in glomeruli No. 6 and No. 7 responding to $\mathrm{O}$ and A differently. If LN1 laterally inhibits both OSN types in glomeruli No. 6 and No. 7, such inhibition should eliminate the differences between the representations of $\mathrm{O}$ and $\mathrm{A}$. Therefore, we make LN1 to specifically connect to glomeruli No. 6 and No. 7 and to have one more arbitrary connection with glomeruli No. 1. The connectivity of LN2 is also arbitrarily set because other theoretical studies have shown that the feedback inhibition via random recurrent connections can increase separability of representations in the PN layer. [55,152-156]. The connectivity of all LNs is listed in Table 5.1.

Surprisingly, although most odor pairs are keeping separated during the olfactory processing in the recurrent networks, the same wiring of connectivity can still generalize the pair of $\mathrm{O}$ and $\mathrm{A}$ as the experimental results in [150] (Fig. 5.5A and 5.5B). In line with our hypothesis, the neural circuits in the predetermined connectivity generalize the pair $\mathrm{O}$ and $\mathrm{A}$ in $\mathrm{PN}$ ensembles although their input odorant-evoked patterns are separated. This odor pair is less separated in the PN activity than it was in the OSN activity. When two odors $\mathrm{O}$ and $\mathrm{A}$ are presented, the activation of LN1, not LN2, attributes to the generalization between $\mathrm{O}$ and A (Fig. 5.5C). The feedback from LN1 inhibits the PN responses in both glomeruli No. 6 and No. 7 eliminating the differences between odor-evoked patterns from input. The neuronal signals of OSNs seem less noisy than those of PNs, but this is not true. This is because the first two principal components only account for $60.57 \%$ variance of original information of OSN signals, and most noise is represented in the third dimension and upward.

These results conclude that patterns in distinct combinatorial codes evoked by different odor quality and heterogeneous recurrent connectivity are crucial factors in modulating the separation between odors. OSN response patterns and connectivity of recurrent networks determine which LNs will be activated and how much their activation strength is. Similarly, the activation strength of these LNs and the same connectivity of recurrent networks determine which PNs will be inhibited and how much strength will suppress these PNs. The interaction between neurons and layers coupling with the heterogeneous connectivity constructs the framework of network dynamics in the antennal lobe. These collective mechanisms relocate odor identities in the odor space and thus modulate representations between different odor qualities.

\subsection{Discussion}

We have demonstrated how the same antennal lobe circuit can serve conflicting neuronal coding strategies - separation and generalization. The same inhibitory connectivity can both increase the separation between some odor pairs and generalize others. Local inhibition in recurrent networks can reduce the PN activity in specific 
glomeruli representing the overlap or the difference between odors. The feedback inhibition on glomeruli in heterogeneous networks is selective and depends on the OSN patterns evoked by different odor qualities and the connections of recurrent networks.

A scenario for separating a pair of odor $\mathrm{O}$ and odor $\mathrm{P}$ is illustrated in Fig. 5.6A. Different colors represent neurons in different glomeruli. Ellipses and rectangles denote OSN ensembles and PN ensembles in individual glomeruli respectively. Filled symbols represent activated neural ensembles. We assume that OSN inputs in at least two glomeruli are needed to activate LNs. The OSN patterns evoked by odor $\mathrm{O}$ and odor $\mathrm{P}$ overlap in the orange and dark green OSNs. The glomeruli where the orange and dark green OSNs project are laterally connected by the brown LN, and the brown LN is then activated by OSN input. In contrast, the glomeruli laterally connected by the gray LN are less overlapped with each OSN pattern, and thus the gray LN cannot be activated by odor $\mathrm{O}$ or odor P. Finally, the responses of the orange and dark green PNs are inhibited because of the feedback inhibition from the brown LN. The patterns between odor $\mathrm{O}$ and odor $\mathrm{P}$ at the $\mathrm{PN}$ level do not overlap, and these odors are more separated during the transformation between the first-order and the second-order neurons.

Interestingly, LNs seem to compete to be activated during the olfactory processing. The selection of winner (e.g. the brown LN in the separation scenario) depends upon the proximity between the connectivity of LNs and OSN response patterns. The overlap between the glomeruli where the brown LN laterally connects and the glomeruli where the OSNs are evoked is more than the overlap between the connectivity of the gray LN and OSN response patterns. As a result, the brown LN is activated, dominating the processing of odor information and decorrelating the pair of odor $\mathrm{O}$ and odor $\mathrm{P}$.

We again use the same recurrent connectivity, but a different odor pair, odor Q and odor R, to show how the generalization can occur in the same wiring of networks. Odor Q evokes the yellow OSNs, not the light green OSNs, and the same OSNs respond to odor $\mathrm{R}$ in the opposite way (Fig.5.6B). Therefore, the OSN pattern evoked by odor $\mathrm{Q}$ is differentiated from that evoked by odor $\mathrm{R}$. On the basis of the same rules in earlier separation scenario, both odors can activate the gray LN, and this LN inhibits the PNs in glomeruli where this LN locally connects. Because of the feedback inhibition, the activity of the yellow PNs and the light green PNs is suppressed. Both odors are now represented by the same pattern at the output level and are thus generalized in neural circuits. Here, we use simple examples to demonstrate how the same wiring of recurrent networks can perform conflict functions - separation and generalization.

In the olfactory system, the representations at the OSN level are redundant [31,74, 88,163]. A study implementing PCA to analyze the OSN responses across different species from insects to mammals, suggesting that merely the first two principal 
A

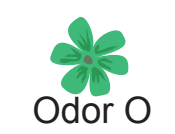

Separation
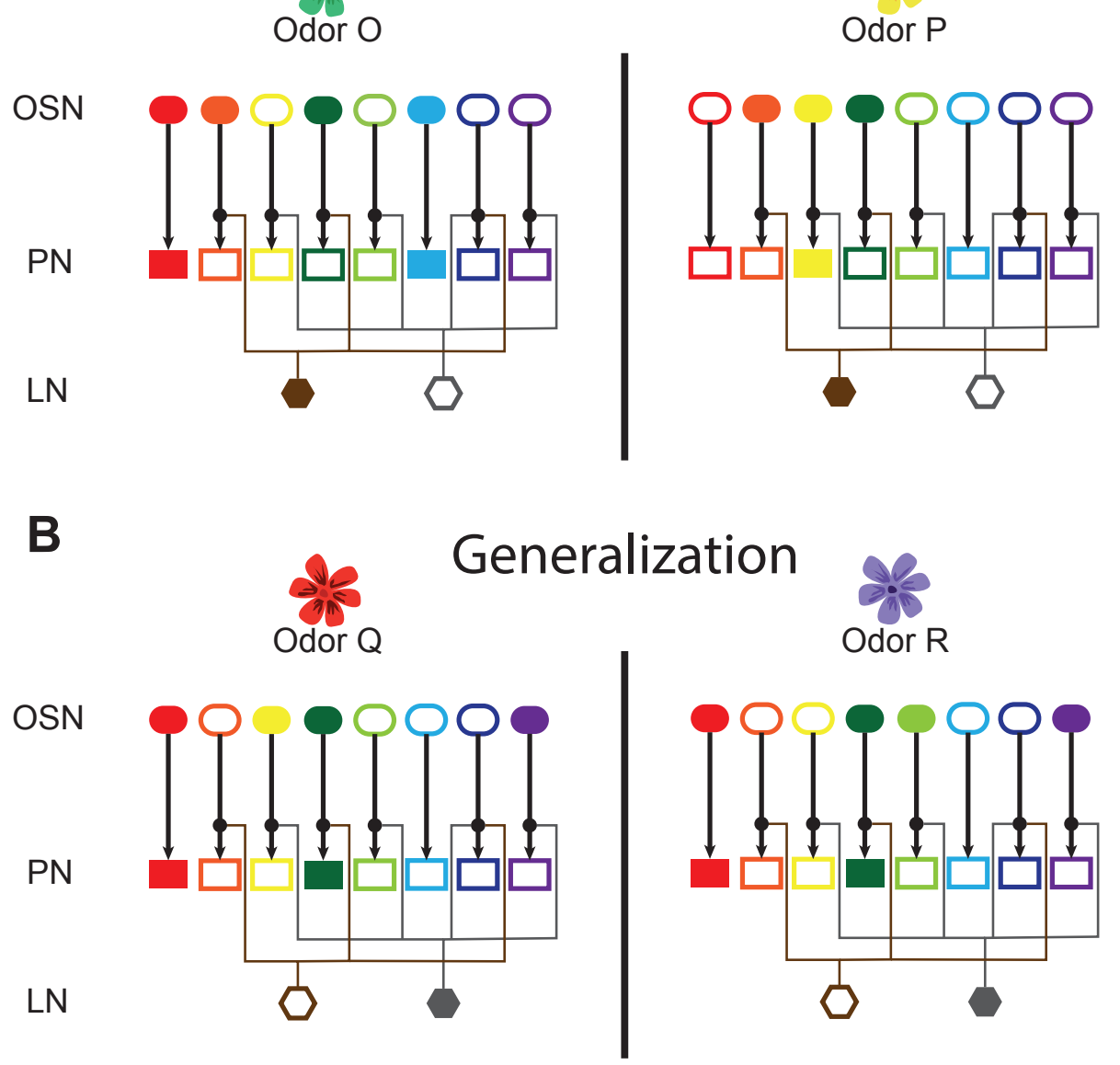

Figure 5.6: Schematic illustration of separation and generalization in the same wiring of networks. Different colors represent neurons in different glomeruli (from left to right: red, orange, yellow, dark green, light green, light blue, dark blue, violet). The brown LN is on the left, and the gray LN is on the right. Ellipses and rectangles denote OSN ensembles and PN ensembles in individual glomeruli respectively. Filled symbols represent activated neural ensembles, and empty one means a silent neural ensemble. In this illustration, we assume that OSN inputs in at least two glomeruli are needed to activate LNs. A. Separation. The OSN patterns evoked by odor O and odor P overlap in the orange and dark green OSNs. The feedback inhibition from the activated brown LN eliminates the responses of the orange PNs and dark green PNs, thus causing this pair more separated. B. Generalization. The differences between odor $\mathrm{Q}$ and odor $\mathrm{R}$ at input are represented in the yellow OSNs and the light green OSNs. The feedback inhibition from the activated gray LN eliminates the responses of the yellow PNs and light green PNs, thus causing this pair generalized.

components can explain half variance of OSN response to odors [164]. Besides, in the same PCA analysis, the first principal component projection of randomly 
sampling $5 \%$ of OSN response data was highly correlated that of all OSN response data. Another study in honeybees suggested that using nearly $25 \%$ of glomerular input activity could predict what odors were given at $85 \%$ success rate [110]. These studies imply that the olfactory system may duplicate response motifs in different OSN types, and these similar responses across OSN types may arise from common evolutionary origins [165]. Another report in Drosophila also showed that different OSN types had highly correlated odor responses [53].

Indeed, odor identity encoded in the redundant combinatorial scheme at OSNs is required for implementing heterogeneous inhibition in modulating separation between odors. The redundancy of encoding odors at OSNs contributes to overlap between input patterns, and the olfactory system can modulate the amount of overlap between odor representations by specific inhibition to vary the distance between identities in the odor space.

When inhibition plays an important role in recurrent networks, the sparse responses of PNs are naturally the product of such inhibition mechanisms. In a recent report studying Drosophila, the authors found that 8 out of 37 PNs were not activated by any odors, and the stimuli set were composed of many and chemically diverse odors [47]. The authors also indicated that in $64 \%$ of all odor responses, PNs were suppressed below the baseline of spike rates. Similar results were found in moth, suggesting that only one out of approximately 20 PNs could be activated by any of seven testing odors [139]. Another report suggested that odors were represented by sparse activity of PN ensembles [12]. The contrast between redundant OSN responses and sparse activity of PNs verifies that the inhibition is active during olfactory processing and serves as the main mechanism in recurrent networks.

Although in this chapter, we only discuss how neural circuits modulate the separation between single odor qualities, it has been found that the separation between odor mixtures was also increased in PNs [138]. The underlying mechanisms of separating odor mixtures are likely similar to the mechanisms applying for single odors because the odor mixtures are also represented in redundant combinatorial codes at the OSN level.

Such olfactory processing in modulating the similarity between representations is the collective consequence of interactions between odor-evoked OSN input and specific feedback inhibition, and such specific inhibition is determined by the interactions between the same above OSN input and the connectivity of local recurrent networks. Yet the morphological development of LN connectivity to specific glomeruli is unknown. However, we argue that the antennal lobe can gain more freedom from unrestricted design of inhibitory spatial patterns than from inherently limited odorant receptor-ligand interactions in the neural computation. 



\section{Discussion and conclusions}

\subsection{Discussion}

\subsubsection{Local computation in olfactory processing}

Our theoretical results in this study demonstrate how heterogeneous lateral inhibition and nonlinear interglomerular transformation simultaneously contribute to three distinct functions in the olfactory processing. In concert with the morphology of network connectivity and the physiological characteristics of olfactory neurons recently discovered in the antennal lobe, our theoretical model for olfactory processing enables putting a collective interpretation of mechanisms in concentration-invariant odor representations, discrete representations for odor mixtures, and olfactory coding in modulating separation between odors.

Remarkably, the abrupt transition between representations at the PN level does not attribute to the significant activity change across all PNs, but to the discontinuous responses of a small subset of PNs. This assumption is supported by the evidence from two different experiments investigating neuronal responses to odor quantity change and to odor morphing in quality $[76,119]$. Different experiment design indicating the same results of local response change at the PN level suggests that the local inhibition should serve as the main mechanism in olfactory processing. If the global inhibition plays an important role, the drastic change of responses should occur in most PNs when inhibition starts to be involved in recurrent networks. Moreover, the abrupt transition between representations for each odor does not happen at the same concentration in increasing odor quantity, and it does not occur for each odor pair at the same mixture ratio in odor morphing either. This may suggest that inhibition strength is heterogeneous and dependent on the odor-evoked patterns representing the quality of input odors or odor mixtures.

The odor information is encoded in different responses of OSN types as combinatorial codes. The odor signals distributed in a number of glomeruli or units allow the antennal lobe to use specific recurrent connections to converge signals from specific units to inhibit targeted output PNs. For example, pentyl acetate and 4-methyl phenol elicit similar OSN responses in VM7 glomerulus, but the presynaptic PNs in the same glomerulus respond distinctly to these two odors [48]. Interestingly, after 
attenuating lateral inhibition by removing the OSNs in other glomeruli, the PNs in VM7 exhibit similar responses to both pentyl acetate and 4-methyl phenol. Therefore, PNs in VM7 glomerulus receive similar feedforward excitatory inputs from their cognate OSNs in two different odors, but the neural circuits can selectively apply lateral inhibition onto these PNs only in a specific odor. This evidence supports that the antennal lobe performs different computation to distinct odors at specific glomeruli, and the inhibition specific to odors is actively implemented in olfactory processing.

When odors are represented by a number of parallel glomeruli or units, the antennal lobe can modulate the separation between odors. Reducing common units of odor representations separates odors further more while attenuating different units between odor representations can generalizes odors. The olfactory circuits may use glomerulus-specific inhibition $[57,58,63,163]$ to perform computation to modulate the distance between a specific odor pair. Although how the morphological development of LN innervation interconnects specific glomeruli is hitherto unknown, the olfactory system should gain more freedom to reshape odor representations from unrestricted design of recurrent connections than inherently limited odorant receptorligand interactions.

In conclusion, the phenomena that olfactory computation occurs in specific and local glomeruli via recurrent connectivity have been long neglected. The specific activation of local neurons inhibiting PNs at specific glomeruli regulates the dynamics of olfactory circuits. Our results suggest that the local and specific inhibition could better explain several underlying mechanisms in the olfactory processing than global inhibition does.

\subsubsection{Interglomerular interactions in olfactory circuits}

There has been a debate on whether the antennal lobe or the olfactory bulb broadens or sharpens the tuning of PNs compared with OSNs. Several studies suggested that lateral inhibition narrowed the tuning of PNs or MCs $[53,57,58,71,166]$ whereas one study held the opposite opinion [47]. Some reports indicated that the response patterns at OSNs and PNs were similar and supported the neutral effects from the functions of the antennal lobe $[10,65]$. If we extend our view from a single glomerulus to more global interglomerular networks, we may answer why these studies appear to contradict each other.

PNs not only receive excitatory input from their presynaptic OSNs but also receive inhibitory input from LNs. For those OSNs responding to an odor, part of their postsynaptic PNs in some glomeruli may respond to this odor while part of other postsynaptic PNs in different glomeruli may be silent because of local inhibition. Similarly, PNs may be faithful to reflect the responses from their presynaptic OSNs 
in some odors whereas they may be silent in other odors although they receive excitatory input from their presynaptic OSNs. This is because these PNs are silenced by inhibitory LNs, and the odor-specific inhibition from LNs abolishes the excitation from their presynaptic OSNs in specific odors. The lateral inhibition thus sharpens the tuning of PNs compared with their presynaptic OSNs.

In contrast, the nonlinear intraglomerular transformation contributes to broaden the tuning of PNs. A few responses at OSNs can be highly boosted at their postsynaptic PNs in the same glomerulus, and thus PNs can respond to more odors than OSNs. Interestingly, different studies sampling different odor-glomerulus combinations naturally saw partial facts of olfactory computation in the olfactory system. We argue that to understanding the interactions between olfactory neurons, we have to consider both intraglomerular and interglomerular relationships and not to only focus on one or a few glomeruli.

\subsubsection{More characteristics of olfactory processing}

The redundancy of encoding odor identity in OSNs is a sine qua non for implementing inhibition in olfactory processing. The amount of olfactory information is reduced during the transformation between the first layer and the second layer in the antennal lobe or the olfactory bulb, and the sparse representations in PNs $[12,47,139]$ are naturally the products of implementing inhibition in neural processing.

The olfactory system can be robust against concentration fluctuation, but at the same time be sensitive to intermediate intensity change in a stimulus. We have showed that the olfactory system can accomplish concentration invariant coding and retrieve significant change in odor quantity, based on two underlying neural mechanisms, nonlinear intraglomerular transformation and local lateral inhibition. Some studies suggested that several fixed point attractors may divide the odor space into several subspaces, and the trajectory of each odor representation converges towards one of the attractors after odor onset $[98,119,167]$. The dynamics of olfactory system is invariant to initial points (invariant to concentration fluctuation), and the trajectories representing similar odors will converge to the same attractor, thus performing concentration-invariant coding. The attractor model also exhibits similar dynamics in odor mixtures so that odor mixtures converge to several attractors and are separated into discrete clusters. Our investigation already indicated that separating and clustering odors mainly result from the lateral inhibition. This implies that the characteristics of attractors should be highly related to how the lateral inhibition emerges in recurrent networks.

The implement of recurrent connectivity in other theoretical studies was based on random networks $[95,96,98,155,156,168]$. To our knowledge, we are the first study 
to investigate the effect of specific connectivity patterns on the antennal lobe. Different connectivity could result in different outcomes in neural circuits. Previous studies using random connectivity may not notice and compare the effect of different connection patterns.

\subsection{Conclusions}

In this thesis, we implement two mechanisms - nonlinear intraglomerular transformation and heterogeneous connectivity of inhibitory LNs to interpret three distinct olfactory strategies in the olfactory modality. The nonlinearity of signal transformation from input OSNs to output PNs is accomplished by the high release probability of vesicles at the synapses between OSNs and PNs, coupling with the synaptic depression caused by the vesicle depletion. Such transformation boosts PN responses to saturation so that similar PN responses contribute to invariant representations. The heterogeneity of local recurrent networks permits the antennal lobe to shape odor representations in specific glomeruli and selectively activate LNs to regulate the dynamics of olfactory circuits. Which LN can be activated depends on the interactions between the input odor-evoked patterns and the connectivity of local recurrent networks.

Although neuronal properties and network connectivity in the antennal lobe are mostly explored, which neural mechanisms underlying the olfactory processing and how the antennal lobe implementing the neural mechanisms remain obscure. Studies investigating the responses of animals to different odor quantity suggested that concentration invariant coding might be implemented in the olfactory system [106, 121-123]. Other studies indicated that odor mixtures are perceived holistically as configural odors, not as several mixed components $[119,138,145,146]$. The role of sensory circuits has been thought to decorrelate input patterns to make efficient use of coding space and to improve odor discrimination $[4,5,19,101,151]$. However, the analysis of our experimental data indicated that the antennal lobe could generalize patterns from distinct odors [150]. Considerable experimental data have revealed the outcomes of olfactory coding, but the underlying mechanisms have not been well explained.

In this study, on the basis of the known facts in olfactory circuits, our model provides new perspectives to explain the observed outcomes of olfactory coding. First, our theoretical results suggest that concentration-invariant representations arise from two phases of PN responses. Inhibitory LNs suppress the activity of PNs when these LNs are activated only at high odor concentrations, thus causing distinct representations at PNs between low and high odor concentrations. In addition, the nonlinear signal transformation between OSNs to their presynaptic PNs boosts PN 
responses to saturation, and the saturating and thus similar responses also contribute to concentration-invariant representations in the olfactory processing.

Second, OSN patterns evoked by odor mixtures and their components could activate different sets of LNs. Therefore, different sets of PNs are inhibited by these individual sets of LNs, and the representations between the mixtures and their components in PNs are distinct and separated. Third, the heterogeneous recurrent connections of networks may explain how conflicting neural coding strategies - separation and generalization can be functioned in the same neural circuits. One odor pair at output becomes more separated if both of them recruit the same feedback inhibition attenuating the overlap between their input patterns. Conversely, another feedback inhibition simultaneously recruited by an odor pair then suppressing the differences between these odors can generalize their representations. Determining which LNs are activated to inhibit PNs in selective glomeruli in which odors depends on the interactions between the input odor-evoked patterns and the connections of recurrent networks.

We try to answer a number of open questions in sensory coding. Our model is not specifically constructed to account for one select data set or one particular mechanism. Instead, our proposed model is capable of explaining three collective phenomena of how olfactory circuits encode quantitative information in individual odors, separate odor mixtures from their components based on qualitative information, and modulate the separation between odor pairs. Finally, we promote the role of LNs historically underrepresented. Together with the LN heterogeneous connectivity of recurrent networks, the olfactory neurons can not only execute one of mysterious olfactory functions but in fact generate all of them - the outcomes depending on odor-evoked input patterns and the wiring of olfactory circuits. 



\section{Summary}
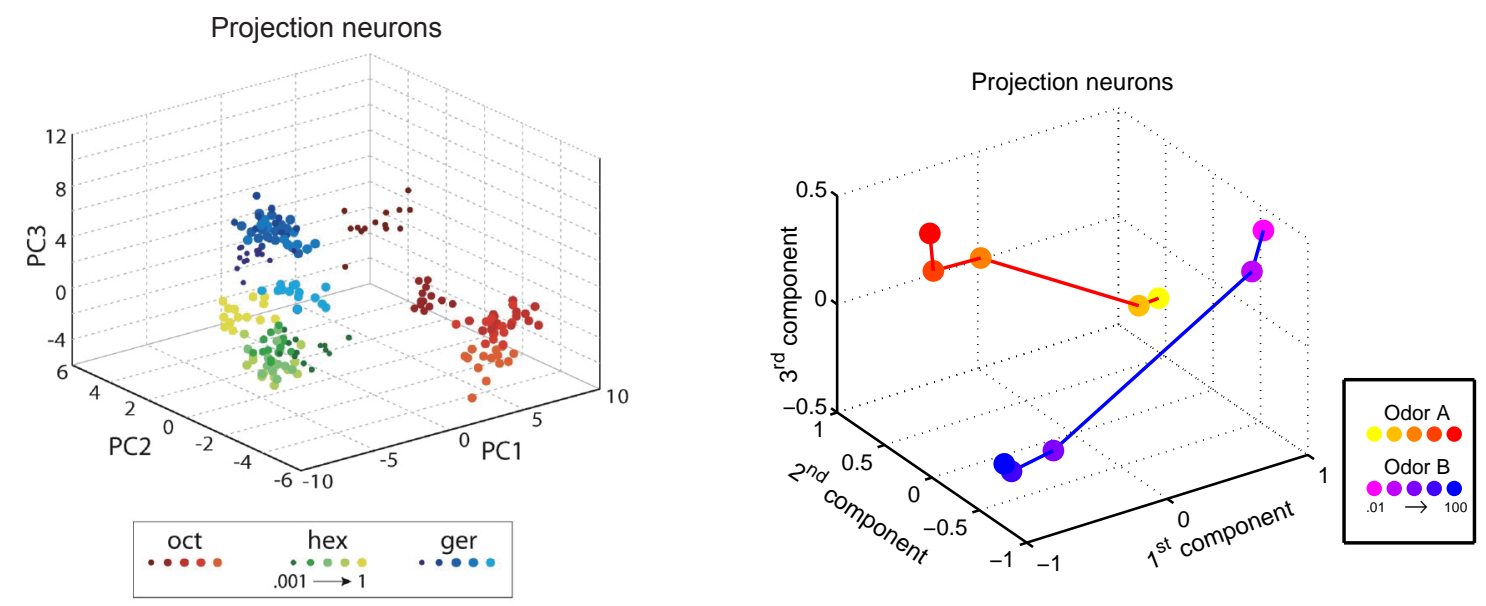

S1. Concentration-invariant representations at output neurons. Left: empirical evidence from [76]; projection neurons in locusts. Right: theoretical evidence based on our model.

The odor representations in sensory input (olfactory sensory neurons, OSNs) depend on the quantity of stimuli. However, the odor signals in the output of the first processing circuit (antennal lobe or olfactory bulb) are transformed into discrete representations invariant to concentration change. The quantitative information is only applied to categorize odors into two discrete clusters, and odors are classified to one cluster representing lower concentrations and another cluster representing higher concentrations. Our theoretical results suggest that such abrupt transition between odor representations arises from the sudden response change of a subset of output neuron (projection neuron, PN). Inhibitory local neurons (LNs) suppress the activity of PNs when these LNs are activated only at higher odor concentrations, thus causing the abrupt change of $\mathrm{PN}$ responses between lower and higher odor concentrations. In addition, the nonlinear signal transformation between OSNs to their presynaptic PNs boosts PN responses to saturation, and the saturating and thus similar responses also contribute to concentration-invariant representations in the olfactory processing. 


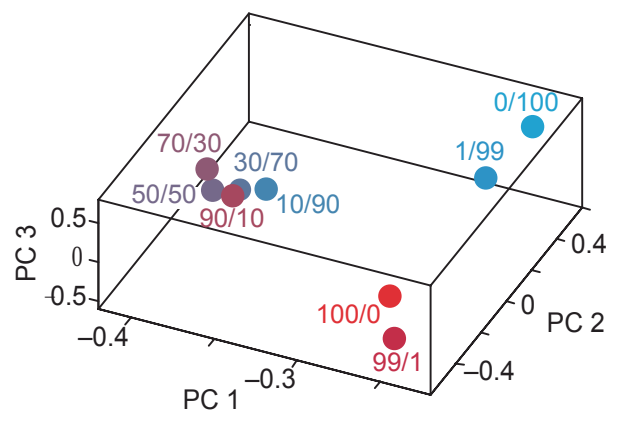

Arg His

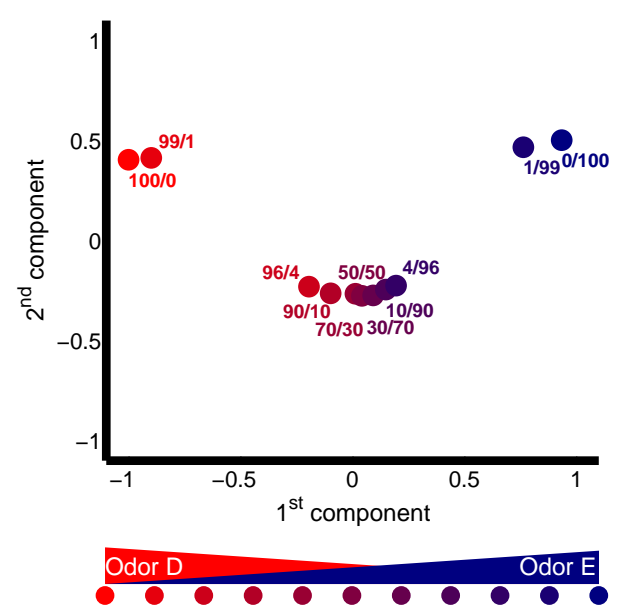

S2. Discrete representations of odor mixtures at output neurons. Left: empirical evidence from [119]; mitral cell in zebrafishes (analogous to projection neurons in insects). Right: theoretical evidence based on our model.

How does the olfactory system evaluate a stimulus consisting of multiple qualities as an odor mixture or as a pure odor contaminated by chemical noise that has to be filtered out in olfactory processing? Why are odor mixtures perceived holistically as configural odors, not as several mixed components? The experimental results suggest that the representations of odor mixtures are separated from those of components in the odor space. The olfactory system considers an odor mixed with a little amount of heterogeneous odorant to be this odor contaminated by chemical noise, and olfactory circuits filter out this noise and represent this odor with its main quality. A possible explanation is that the generalization between a component and this component mixed with a little amount of the second component is because both odors at similar concentration ratios activate the same inhibitory LNs, and PN responses are then suppressed by the same specific inhibition. Therefore, the representations between both odors in PNs are similar. On the ground of heterogeneous connectivity of LNs, new inhibitory LNs will be recruited to suppress different PN ensembles when the concentration of the second component in odor mixtures increases, and the representations between the first component and intermediate mixtures in PNs are thus distinct and separated. 

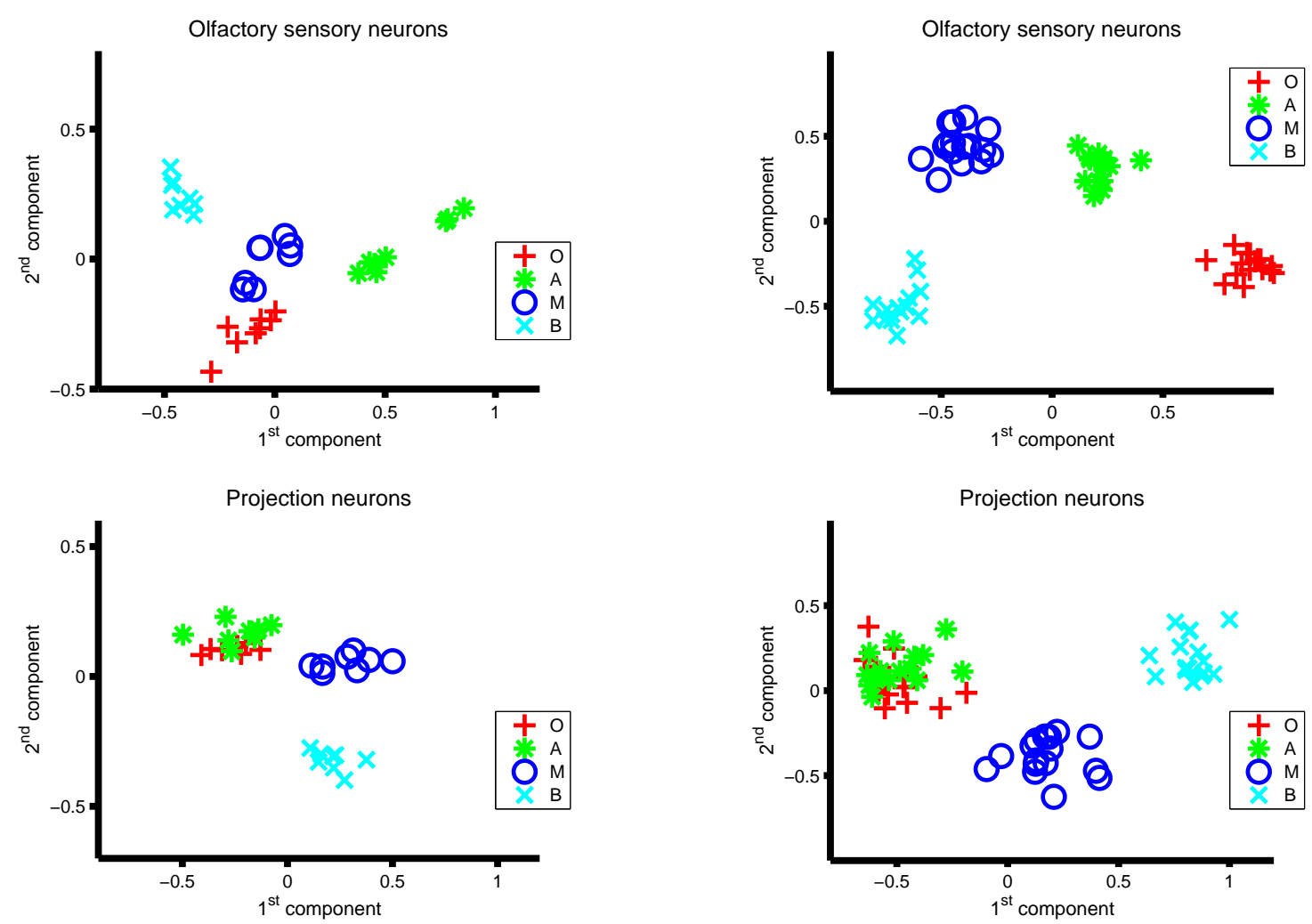

S3. Modulating separation between odors. Left: empirical evidence from [150]; olfactory sensory neurons and projection neurons in Drosophila. Right: theoretical evidence based on our model.

It has long been thought that the antennal lobe or the olfactory bulb enhances odor discrimination and increases separation between olfactory inputs from OSNs. Such mechanisms may result from inhibitory recurrent networks. Our analysis of experimental data suggests that the neuronal signals of one odor pair are similar in PN ensembles although the odorant-evoked patterns of this pair in OSN ensembles are distinct, and thus this odor pair is less separated in PN activity than it was in OSN activity. The heterogeneous recurrent connections of networks may explain how conflicting neural coding strategies - separation and generalization can be functioned in the same neural circuits. One odor pair at output becomes more separated if both of them recruit the same feedback inhibition attenuating the overlap between their input patterns. Conversely, another feedback inhibition simultaneously recruited by an odor pair then suppressing the differences between these odors can generalize their representations. Determining which LNs are activated to inhibit specific PN ensembles in which odors depends on the interactions between the input odor-evoked patterns and the connections of recurrent networks. 



\section{Acknowledgments}

First of all, I am deeply indebted to Dr. Marc Timme, who gives me the possibility to join his outstanding research group and to participate the project investigating the network dynamics of olfactory systems. There are a lot of open questions and possibility in this project, and I really enjoy doing research on this topic. He is a brilliant scientist and always makes an enlightened approach to answer my questions. He is also approachable and is willing to spend time instructing me in doing research. I am always inspired by his enthusiasm and passion for research.

I am grateful to Dr. André Fiala for his advice on my project from his abundant knowledge and direct insight about biology. I enjoy discussing with him about my project and also obtain useful suggestions. He is always friendly and prompts to reply me when I ask him questions via e-mail.

I would like to thank Dr. Ulrich Parlitz for his constructive opinions pointing out the prospect or the weakness of my project. I also thank him for his helpful advice on communication skills in making oral presentations.

I would like to thank the Max Planck society for spending large sums of money purchasing the access to many databases of scientific literature. I can study the original contributions of many scientists which provide solid foundations of my research.

I would like to thank the Göttingen Graduate School for Neurosciences, Biophysics, and Molecular Biosciences (GGNB) for providing financial supports for my $\mathrm{PhD}$ project and conference attendance. I also learned much and diverse knowledge from many methods and skills courses provided by GGNB.

I would like to thank all partners including Secretary Barbara Kutz in the Network Dynamics group. They are so nice to help me solve many problems including science, personal matters, and even German translation.

I am very grateful to my parents by dedicating my $\mathrm{PhD}$ dissertation to them. Without their constant and great support, I cannot be here to engage in doing fascinating scientific research.

Last but not least, I am very grateful to my wife by dedicating my PhD dissertation to her. She is always willing to look after me every day and to prepare authentic and delicious Taiwanese food which really warms up my stomach as well as my 
heart! She is always accompanying me while I am doing research and writing this dissertation. Without her, I cannot finish my dissertation. 


\section{Bibliography}

[1] E. R. Kandel, J. H. Schwartz, T. M. Jessell et al., Principles of neural science. McGraw-Hill New York, 2000, vol. 4.

[2] E. T. Rolls and G. Deco, Computational neuroscience of vision. Oxford university press Oxford, 2002.

[3] R. Fettiplace and C. M. Hackney, "The sensory and motor roles of auditory hair cells," Nat Rev Neurosci, vol. 7, no. 1, pp. 19-29, 2006.

[4] R. I. Wilson and Z. F. Mainen, "Early events in olfactory processing," Annu Rev Neurosci, vol. 29, pp. 163-201, 2006.

[5] R. W. Friedrich, "Neuronal computations in the olfactory system of zebrafish," Annu Rev Neurosci, vol. 36, pp. 383-402, 2013.

[6] R. Axel, "The molecular logic of smell," Scientific American, vol. 273, no. 4, pp. 154-159, 1995.

[7] L. B. Buck, "The molecular architecture of odor and pheromone sensing in mammals," Cell, vol. 100, no. 6, pp. 611-618, 2000.

[8] P. A. Godfrey, B. Malnic, and L. B. Buck, "The mouse olfactory receptor gene family," Proc Natl Acad Sci U S A, vol. 101, no. 7, pp. 2156-61, 2004.

[9] A. Fiala, T. Spall, S. Diegelmann, B. Eisermann, S. Sachse, J. M. Devaud, E. Buchner, and C. G. Galizia, "Genetically expressed cameleon in Drosophila melanogaster is used to visualize olfactory information in projection neurons," Curr Biol, vol. 12, no. 21, pp. 1877-84, 2002.

[10] J. W. Wang, A. M. Wong, J. Flores, L. B. Vosshall, and R. Axel, "Two-photon calcium imaging reveals an odor-evoked map of activity in the fly brain," Cell, vol. 112, no. 2, pp. 271-82, 2003.

[11] R. F. Stocker, G. Heimbeck, N. Gendre, and J. S. de Belle, "Neuroblast ablation in Drosophila P[GAL4] lines reveals origins of olfactory interneurons," $J$ Neurobiol, vol. 32, no. 5, pp. 443-56, 1997.

[12] C. M. Root, J. L. Semmelhack, A. M. Wong, J. Flores, and J. W. Wang, "Propagation of olfactory information in Drosophila," Proceedings of the National Academy of Sciences, vol. 104, no. 28, pp. 11826-31, 2007.

[13] A. M. Packer, B. Roska, and M. Hausser, "Targeting neurons and photons for optogenetics," Nat Neurosci, vol. 16, no. 7, pp. 805-815, 2013.

[14] C. G. Galizia and W. Rossler, "Parallel olfactory systems in insects: anatomy and function," Annu Rev Entomol, vol. 55, pp. 399-420, 2010. 
[15] R. I. Wilson, "Understanding the functional consequences of synaptic specialization: insight from the Drosophila antennal lobe," Curr Opin Neurobiol, vol. 21, no. 2, pp. 254-60, 2011.

[16] H. M. Robertson, C. G. Warr, and J. R. Carlson, "Molecular evolution of the insect chemoreceptor gene superfamily in Drosophila melanogaster," Proc Natl Acad Sci U S A, vol. 100 Suppl 2, pp. 14 537-42, 2003.

[17] C. I. Bargmann, "Comparative chemosensation from receptors to ecology," Nature, vol. 444, no. 7117, pp. 295-301, 2006.

[18] A. Fiala, "Olfaction and olfactory learning in Drosophila: recent progress," Curr Opin Neurobiol, vol. 17, no. 6, pp. 720-6, 2007.

[19] N. Y. Masse, G. C. Turner, and G. S. Jefferis, "Olfactory information processing in Drosophila," Curr Biol, vol. 19, no. 16, pp. R700-13, 2009.

[20] H. M. Robertson and K. W. Wanner, "The chemoreceptor superfamily in the honey bee, Apis mellifera: expansion of the odorant, but not gustatory, receptor family," Genome Res, vol. 16, no. 11, pp. 1395-403, 2006.

[21] D. Müller-Schwarze, Chemical ecology of vertebrates. Cambridge, New York: Cambridge University Press, 2006.

[22] R. F. Stocker, M. C. Lienhard, A. Borst, and K. F. Fischbach, "Neuronal architecture of the antennal lobe in Drosophila melanogaster," Cell and Tissue Research, vol. 262, no. 1, pp. 9-34, 1990.

[23] R. F. Stocker, "The organization of the chemosensory system in Drosophila melanogaster: a review," Cell Tissue Res, vol. 275, no. 1, pp. 3-26, 1994.

[24] E. A. Hallem and J. R. Carlson, "The odor coding system of Drosophila," Trends Genet, vol. 20, no. 9, pp. 453-9, 2004.

[25] A. Couto, M. Alenius, and B. J. Dickson, "Molecular, anatomical, and functional organization of the Drosophila olfactory system," Curr Biol, vol. 15, no. 17 , pp. 1535-47, 2005.

[26] E. Fishilevich and L. B. Vosshall, "Genetic and functional subdivision of the Drosophila antennal lobe," Curr Biol, vol. 15, no. 17, pp. 1548-53, 2005.

[27] M. L. Spletter and L. Luo, "A new family of odorant receptors in Drosophila," Cell, vol. 136, no. 1, pp. 23-5, 2009.

[28] A. Chess, I. Simon, H. Cedar, and R. Axel, "Allelic inactivation regulates olfactory receptor gene expression," Cell, vol. 78, no. 5, pp. 823-834, 1994.

[29] B. Malnic, J. Hirono, T. Sato, and L. B. Buck, "Combinatorial receptor codes for odors," Cell, vol. 96, no. 5, pp. 713-23, 1999.

[30] L. B. Vosshall, A. M. Wong, and R. Axel, "An olfactory sensory map in the fly brain," Cell, vol. 102, no. 2, pp. 147-59, 2000.

[31] G. Laurent, "A systems perspective on early olfactory coding," Science, vol. 286, no. 5440, pp. 723-8, 1999.

[32] Y. H. Chou, M. L. Spletter, E. Yaksi, J. C. Leong, R. I. Wilson, and L. Luo, "Diversity and wiring variability of olfactory local interneurons in the Drosophila antennal lobe," Nat Neurosci, vol. 13, no. 4, pp. 439-49, 2010. 
[33] G. Arnold, C. Masson, and S. Budharugsa, "Comparative study of the antennal lobes and their afferent pathway in the worker bee and the drone (Apis mellifera)," Cell and Tissue Research, vol. 242, no. 3, pp. 593-605, 1985.

[34] C. G. Galizia, S. L. McIlwrath, and R. Menzel, "A digital three-dimensional atlas of the honeybee antennal lobe based on optical sections acquired by confocal microscopy," Cell Tissue Res, vol. 295, no. 3, pp. 383-94, 1999.

[35] P. Mombaerts, "Axonal wiring in the mouse olfactory system," Annu Rev Cell Dev Biol, vol. 22, pp. 713-37, 2006.

[36] Q. Gao, B. Yuan, and A. Chess, "Convergent projections of Drosophila olfactory neurons to specific glomeruli in the antennal lobe," Nat Neurosci, vol. 3, no. 8, pp. 780-5, 2000.

[37] R. Benton, K. S. Vannice, C. Gomez-Diaz, and L. B. Vosshall, "Variant ionotropic glutamate receptors as chemosensory receptors in Drosophila," Cell, vol. 136, no. 1, pp. 149-62, 2009.

[38] A. F. Silbering, R. Rytz, Y. Grosjean, L. Abuin, P. Ramdya, G. S. Jefferis, and R. Benton, "Complementary function and integrated wiring of the evolutionarily distinct Drosophila olfactory subsystems," J Neurosci, vol. 31, no. 38, pp. $13357-75,2011$.

[39] E. A. Hallem and J. R. Carlson, "Coding of odors by a receptor repertoire," Cell, vol. 125, no. 1, pp. 143-60, 2006.

[40] C. A. Yao, R. Ignell, and J. R. Carlson, "Chemosensory coding by neurons in the coeloconic sensilla of the Drosophila antenna," J Neurosci, vol. 25, no. 37, pp. 8359-67, 2005.

[41] T. Miyamoto, Y. Chen, J. Slone, and H. Amrein, "Identification of a Drosophila glucose receptor using $\mathrm{ca}^{2+}$ imaging of single chemosensory neurons," PLoS One, vol. 8, no. 2, p. e56304, 2013.

[42] S. R. Olsen, V. Bhandawat, and R. I. Wilson, "Excitatory interactions between olfactory processing channels in the Drosophila antennal lobe," Neuron, vol. 54, no. 1, pp. 89-103, 2007.

[43] Y. Shang, A. Claridge-Chang, L. Sjulson, M. Pypaert, and G. Miesenbock, "Excitatory local circuits and their implications for olfactory processing in the fly antennal lobe," Cell, vol. 128, no. 3, pp. 601-12, 2007.

[44] H. Kazama and R. I. Wilson, "Homeostatic matching and nonlinear amplification at identified central synapses," Neuron, vol. 58, no. 3, pp. 401-13, 2008.

[45] N. W. Gouwens and R. I. Wilson, "Signal propagation in Drosophila central neurons," J Neurosci, vol. 29, no. 19, pp. 6239-49, 2009.

[46] C. Y. Su, K. Menuz, and J. R. Carlson, "Olfactory perception: receptors, cells, and circuits," Cell, vol. 139, no. 1, pp. 45-59, 2009.

[47] R. I. Wilson, G. C. Turner, and G. Laurent, "Transformation of olfactory representations in the Drosophila antennal lobe," Science, vol. 303, no. 5656, pp. 366-70, 2004.

[48] S. R. Olsen and R. I. Wilson, "Lateral presynaptic inhibition mediates gain control in an olfactory circuit," Nature, vol. 452, no. 7190, pp. 956-60, 2008. 
[49] J. P. McGann, N. Pirez, M. A. Gainey, C. Muratore, A. S. Elias, and M. Wachowiak, "Odorant representations are modulated by intra- but not interglomerular presynaptic inhibition of olfactory sensory neurons," Neuron, vol. 48, no. 6, pp. 1039-53, 2005.

[50] G. J. Murphy, D. P. Darcy, and J. S. Isaacson, "Intraglomerular inhibition: signaling mechanisms of an olfactory microcircuit," Nat Neurosci, vol. 8, no. 3, pp. 354-64, 2005.

[51] M. Wachowiak, J. P. McGann, P. M. Heyward, Z. Shao, A. C. Puche, and M. T. Shipley, "Inhibition of olfactory receptor neuron input to olfactory bulb glomeruli mediated by suppression of presynaptic calcium influx," J Neurophysiol, vol. 94, no. 4, pp. 2700-12, 2005.

[52] D. Vucinic, L. B. Cohen, and E. K. Kosmidis, "Interglomerular centersurround inhibition shapes odorant-evoked input to the mouse olfactory bulb in vivo," J Neurophysiol, vol. 95, no. 3, pp. 1881-7, 2006.

[53] V. Bhandawat, S. R. Olsen, N. W. Gouwens, M. L. Schlief, and R. I. Wilson, "Sensory processing in the Drosophila antennal lobe increases reliability and separability of ensemble odor representations," Nat Neurosci, vol. 10, no. 11, pp. 1474-82, 2007.

[54] A. F. Silbering and C. G. Galizia, "Processing of odor mixtures in the Drosophila antennal lobe reveals both global inhibition and glomerulus-specific interactions," J Neurosci, vol. 27, no. 44, pp. 11966-77, 2007.

[55] S. R. Olsen, V. Bhandawat, and R. I. Wilson, "Divisive normalization in olfactory population codes," Neuron, vol. 66, no. 2, pp. 287-99, 2010.

[56] C. Fonta, X.-J. Sun, and C. Masson, "Morphology and spatial distribution of bee antennal lobe interneurones responsive to odours," Chemical Senses, vol. 18, no. 2, pp. 101-119, 1993.

[57] S. Sachse and C. G. Galizia, "Role of inhibition for temporal and spatial odor representation in olfactory output neurons: a calcium imaging study," $J$ Neurophysiol, vol. 87, no. 2, pp. 1106-17, 2002.

[58] A. F. Silbering, R. Okada, K. Ito, and C. G. Galizia, "Olfactory information processing in the Drosophila antennal lobe: anything goes?" J Neurosci, vol. 28, no. 49, pp. 13075-87, 2008.

[59] R. Okada, T. Awasaki, and K. Ito, "Gamma-aminobutyric acid (GABA)mediated neural connections in the Drosophila antennal lobe," J Comp Neurol, vol. 514, no. 1, pp. 74-91, 2009.

[60] N. K. Tanaka, K. Ito, and M. Stopfer, "Odor-evoked neural oscillations in Drosophila are mediated by widely branching interneurons," J Neurosci, vol. 29, no. 26, pp. 8595-603, 2009.

[61] Y. Seki, J. Rybak, D. Wicher, S. Sachse, and B. S. Hansson, "Physiological and morphological characterization of local interneurons in the Drosophila antennal lobe," J Neurophysiol, vol. 104, no. 2, pp. 1007-19, 2010.

[62] D. Flanagan and A. R. Mercer, "An atlas and 3-D reconstruction of the antennal lobes in the worker honey bee, Apis mellifera L.(Hymenoptera:Apidae)," 
International Journal of Insect Morphology and Embryology, vol. 18, no. 2, pp. 145-159, 1989.

[63] R. I. Wilson and G. Laurent, "Role of GABAergic inhibition in shaping odorevoked spatiotemporal patterns in the Drosophila antennal lobe," J Neurosci, vol. 25, no. 40, pp. 9069-79, 2005.

[64] C. C. Girardin, S. Kreissl, and C. G. Galizia, "Inhibitory connections in the honeybee antennal lobe are spatially patchy," J Neurophysiol, vol. 109, no. 2, pp. 332-43, 2013.

[65] M. Ng, R. D. Roorda, S. Q. Lima, B. V. Zemelman, P. Morcillo, and G. Miesenbock, "Transmission of olfactory information between three populations of neurons in the antennal lobe of the fly," Neuron, vol. 36, no. 3, pp. 463-74, 2002.

[66] A. Acebes, A. Martin-Pena, V. Chevalier, and A. Ferrus, "Synapse loss in olfactory local interneurons modifies perception," J Neurosci, vol. 31, no. 8, pp. 2734-45, 2011.

[67] M. Luo and L. C. Katz, "Response correlation maps of neurons in the mammalian olfactory bulb," Neuron, vol. 32, no. 6, pp. 1165-79, 2001.

[68] J. L. Aungst, P. M. Heyward, A. C. Puche, S. V. Karnup, A. Hayar, G. Szabo, and M. T. Shipley, "Centre-surround inhibition among olfactory bulb glomeruli," Nature, vol. 426, no. 6967, pp. 623-9, 2003.

[69] A. L. Fantana, E. R. Soucy, and M. Meister, "Rat olfactory bulb mitral cells receive sparse glomerular inputs," Neuron, vol. 59, no. 5, pp. 802-14, 2008.

[70] D. H. Kim, M. E. Phillips, A. Y. Chang, H. K. Patel, K. T. Nguyen, and D. C. Willhite, "Lateral connectivity in the olfactory bulb is sparse and segregated," Front Neural Circuits, vol. 5, p. 5, 2011.

[71] C. Linster, S. Sachse, and C. G. Galizia, "Computational modeling suggests that response properties rather than spatial position determine connectivity between olfactory glomeruli," J Neurophysiol, vol. 93, no. 6, pp. 3410-7, 2005.

[72] T. A. Cleland and P. Sethupathy, "Non-topographical contrast enhancement in the olfactory bulb," BMC Neurosci, vol. 7, p. 7, 2006.

[73] P. M. Lledo, G. Gheusi, and J. D. Vincent, "Information processing in the mammalian olfactory system," Physiol Rev, vol. 85, no. 1, pp. 281-317, 2005.

[74] G. Laurent, "Olfactory network dynamics and the coding of multidimensional signals," Nat Rev Neurosci, vol. 3, no. 11, pp. 884-95, 2002.

[75] S. Sachse and C. G. Galizia, "The coding of odour-intensity in the honeybee antennal lobe: local computation optimizes odour representation," Eur $J$ Neurosci, vol. 18, no. 8, pp. 2119-32, 2003.

[76] M. Stopfer, V. Jayaraman, and G. Laurent, "Intensity versus identity coding in an olfactory system," Neuron, vol. 39, no. 6, pp. 991-1004, 2003.

[77] J. P. Martin, A. Beyerlein, A. M. Dacks, C. E. Reisenman, J. A. Riffell, H. Lei, and J. G. Hildebrand, "The neurobiology of insect olfaction: sensory processing in a comparative context," Prog Neurobiol, vol. 95, no. 3, pp. 427-47, 2011. 
[78] H. Spors, D. F. Albeanu, V. N. Murthy, D. Rinberg, N. Uchida, M. Wachowiak, and R. W. Friedrich, "Illuminating vertebrate olfactory processing," J Neurosci, vol. 32, no. 41, pp. 14102-8, 2012.

[79] J. L. Price and T. P. Powell, "The synaptology of the granule cells of the olfactory bulb," J Cell Sci, vol. 7, no. 1, pp. 125-55, 1970.

[80] L. Dryer and P. P. Graziadei, "Mitral cell dendrites: a comparative approach," Anat Embryol (Berl), vol. 189, no. 2, pp. 91-106, 1994.

[81] P. Mombaerts, "Targeting olfaction," Curr Opin Neurobiol, vol. 6, no. 4, pp. 481-6, 1996.

[82] T. W. Chen, B. J. Lin, and D. Schild, "Odor coding by modules of coherent mitral/tufted cells in the vertebrate olfactory bulb," Proc Natl Acad Sci U S A, vol. 106, no. 7, pp. 2401-6, 2009.

[83] J. G. Hildebrand and G. M. Shepherd, "Mechanisms of olfactory discrimination: converging evidence for common principles across phyla," Annu Rev Neurosci, vol. 20, pp. 595-631, 1997.

[84] H. L. Eisthen, "Why are olfactory systems of different animals so similar?" Brain Behav Evol, vol. 59, no. 5-6, pp. 273-93, 2002.

[85] B. W. Ache and J. M. Young, "Olfaction: diverse species, conserved principles," Neuron, vol. 48, no. 3, pp. 417-30, 2005.

[86] U. B. Kaupp, "Olfactory signalling in vertebrates and insects: differences and commonalities," Nat Rev Neurosci, vol. 11, no. 3, pp. 188-200, 2010.

[87] C. Linster and T. A. Cleland, "Decorrelation of odor representations via spike timing-dependent plasticity," Front Comput Neurosci, vol. 4, p. 157, 2010.

[88] C. G. Galizia and R. Menzel, "The role of glomeruli in the neural representation of odours: results from optical recording studies," Journal of Insect Physiology, vol. 47, no. 2, pp. 115-130, 2001.

[89] W. B. Floriano, N. Vaidehi, and W. A. Goddard, "Making sense of olfaction through predictions of the 3-D structure and function of olfactory receptors," Chemical Senses, vol. 29, no. 4, pp. 269-290, 2004.

[90] E. A. Hallem, M. G. Ho, and J. R. Carlson, "The molecular basis of odor coding in the Drosophila antenna," Cell, vol. 117, no. 7, pp. 965-79, 2004.

[91] R. G. Gallager, Discrete stochastic processes. Boston: Kluwer Academic Publishers, 1996.

[92] P. Dayan and L. F. Abbott, Theoretical neuroscience: computational and mathematical modeling of neural systems. Cambridge, MA: MIT press, 2001.

[93] W. Gerstner and W. M. Kistler, Spiking neuron models: Single neurons, populations, plasticity. Cambridge university press, 2002.

[94] M. Oizumi, R. Satoh, H. Kazama, and M. Okada, "Functional differences between global pre- and postsynaptic inhibition in the Drosophila olfactory circuit," Front Comput Neurosci, vol. 6, p. 14, 2012.

[95] A. V. Rangan, "Functional roles for synaptic-depression within a model of the fly antennal lobe," PLoS Comput Biol, vol. 8, no. 8, p. e1002622, 2012. 
[96] M. J. Patel, A. V. Rangan, and D. Cai, "Coding of odors by temporal binding within a model network of the locust antennal lobe," Front Comput Neurosci, vol. 7, p. 50, 2013.

[97] A. N. Burkitt, "A review of the integrate-and-fire neuron model: I. homogeneous synaptic input," Biol Cybern, vol. 95, no. 1, pp. 1-19, 2006.

[98] C. L. Buckley and T. Nowotny, "Transient dynamics between displaced fixed points: an alternate nonlinear dynamical framework for olfaction," Brain Res, vol. 1434, pp. 62-72, 2012.

[99] B. Katz, The release of neural transmitter substances. Springfield, IL: Thomas, 1969.

[100] M. Bazhenov, M. Stopfer, M. Rabinovich, R. Huerta, H. D. Abarbanel, T. J. Sejnowski, and G. Laurent, "Model of transient oscillatory synchronization in the locust antennal lobe," Neuron, vol. 30, no. 2, pp. 553-67, 2001.

[101] M. Yokoi, K. Mori, and S. Nakanishi, "Refinement of odor molecule tuning by dendrodendritic synaptic inhibition in the olfactory bulb," Proc Natl Acad Sci $U S A$, vol. 92, no. 8, pp. 3371-5, 1995.

[102] G. J. Murphy, L. L. Glickfeld, Z. Balsen, and J. S. Isaacson, "Sensory neuron signaling to the brain: properties of transmitter release from olfactory nerve terminals," J Neurosci, vol. 24, no. 12, pp. 3023-30, 2004.

[103] A. A. Biro, N. B. Holderith, and Z. Nusser, "Quantal size is independent of the release probability at hippocampal excitatory synapses," J Neurosci, vol. 25, no. 1, pp. 223-32, 2005.

[104] H. Gu and D. K. O'Dowd, "Cholinergic synaptic transmission in adult Drosophila kenyon cells in situ," J Neurosci, vol. 26, no. 1, pp. 265-72, 2006.

[105] S. A. Kreher, D. Mathew, J. Kim, and J. R. Carlson, "Translation of sensory input into behavioral output via an olfactory system," Neuron, vol. 59, no. 1, pp. 110-24, 2008.

[106] K. Asahina, M. Louis, S. Piccinotti, and L. B. Vosshall, "A circuit supporting concentration-invariant odor perception in Drosophila," J Biol, vol. 8, no. 1, p. 9, 2009 .

[107] J. L. Semmelhack and J. W. Wang, "Select Drosophila glomeruli mediate innate olfactory attraction and aversion," Nature, vol. 459, no. 7244, pp. 21823, 2009 .

[108] M. Wachowiak, L. B. Cohen, and M. R. Zochowski, "Distributed and concentration-invariant spatial representations of odorants by receptor neuron input to the turtle olfactory bulb," J Neurophysiol, vol. 87, no. 2, pp. 1035-45, 2002.

[109] M. de Bruyne, P. J. Clyne, and J. R. Carlson, "Odor coding in a model olfactory organ: the Drosophila maxillary palp," J Neurosci, vol. 19, no. 11, pp. 4520-32, 1999.

[110] C. G. Galizia, S. Sachse, A. Rappert, and R. Menzel, "The glomerular code for odor representation is species specific in the honeybee Apis mellifera," Nat Neurosci, vol. 2, no. 5, pp. 473-478, 1999. 
[111] N. Uchida, Y. K. Takahashi, M. Tanifuji, and K. Mori, "Odor maps in the mammalian olfactory bulb: domain organization and odorant structural features," Nat Neurosci, vol. 3, no. 10, pp. 1035-43, 2000.

[112] M. de Bruyne, K. Foster, and J. R. Carlson, "Odor coding in the Drosophila antenna," Neuron, vol. 30, no. 2, pp. 537-52, 2001.

[113] M. Falasconi, A. Gutierrez-Galvez, M. Leon, B. A. Johnson, and S. Marco, "Cluster analysis of rat olfactory bulb responses to diverse odorants," Chem Senses, vol. 37, no. 7, pp. 639-53, 2012.

[114] B. Malnic, P. A. Godfrey, and L. B. Buck, "The human olfactory receptor gene family," Proceedings of the National Academy of Sciences of the United States of America, vol. 101, no. 8, pp. 2584-2589, 2004.

[115] Y. Niimura and M. Nei, "Evolution of olfactory receptor genes in the human genome," Proc Natl Acad Sci US A, vol. 100, no. 21, pp. 12 235-40, 2003.

[116] R. G. S. P. Consortium, "Genome sequence of the brown norway rat yields insights into mammalian evolution," Nature, vol. 428, no. 6982, pp. 493-521, 2004.

[117] J. Murlis, J. S. Elkinton, and R. T. Cardé, "Odor plumes and how insects use them," Annual Review of Entomology, vol. 37, no. 1, pp. 505-532, 1992.

[118] J. Riffell, L. Abrell, and J. Hildebrand, "Physical processes and real-time chemical measurement of the insect olfactory environment," Journal of Chemical Ecology, vol. 34, no. 7, pp. 837-853, 2008.

[119] J. Niessing and R. W. Friedrich, "Olfactory pattern classification by discrete neuronal network states," Nature, vol. 465, no. 7294, pp. 47-52, 2010.

[120] B. A. Johnson and M. Leon, "Modular representations of odorants in the glomerular layer of the rat olfactory bulb and the effects of stimulus concentration," J Comp Neurol, vol. 422, no. 4, pp. 496-509, 2000.

[121] T. A. Cleland, B. A. Johnson, M. Leon, and C. Linster, "Relational representation in the olfactory system," Proc Natl Acad Sci U S A, vol. 104, no. 6, pp. 1953-8, 2007.

[122] N. Uchida and Z. F. Mainen, "Odor concentration invariance by chemical ratio coding," Frontiers in Systems Neuroscience, vol. 2, 2007.

[123] G. A. Wright, M. G. Thomson, and B. H. Smith, "Odour concentration affects odour identity in honeybees," Proc Biol Sci, vol. 272, no. 1579, pp. 2417-22, 2005.

[124] T. A. Cleland, S. Y. Chen, K. W. Hozer, H. N. Ukatu, K. J. Wong, and F. Zheng, "Sequential mechanisms underlying concentration invariance in biological olfaction," Front Neuroeng, vol. 4, p. 21, 2011.

[125] S. Haykin, Neural Networks: A Comprehensive Foundation, 2nd ed. Upper Saddle River, NJ, USA: Prentice Hall PTR, 1998.

[126] R. W. Moncrieff, The chemical senses, 3rd ed. London: L. Hill, 1967.

[127] R. Gross-Isseroff and D. Lancet, "Concentration-dependent changes of perceived odor quality," Chemical Senses, vol. 13, no. 2, pp. 191-204, 1988. 
[128] M. Bazhenov, M. Stopfer, M. Rabinovich, R. Huerta, H. D. Abarbanel, T. J. Sejnowski, and G. Laurent, "Model of transient oscillatory synchronization in the locust antennal lobe," Neuron, vol. 30, no. 2, pp. 553-67, 2001.

[129] H. Lei, T. A. Christensen, and J. G. Hildebrand, "Local inhibition modulates odor-evoked synchronization of glomerulus-specific output neurons," Nat Neurosci, vol. 5, no. 6, pp. 557-65, 2002.

[130] M. Wachowiak and L. B. Cohen, "Representation of odorants by receptor neuron input to the mouse olfactory bulb," Neuron, vol. 32, no. 4, pp. 723-35, 2001.

[131] J. J. Shynk, Probability, Random Variables, and Random Processes: Theory and Signal Processing Applications. John Wiley \& Sons, 2012.

[132] J. P. Rospars, P. Lansky, H. C. Tuckwell, and A. Vermeulen, "Coding of odor intensity in a steady-state deterministic model of an olfactory receptor neuron," J Comput Neurosci, vol. 3, no. 1, pp. 51-72, 1996.

[133] J. Esslen and K.-E. Kaissling, "Zahl und verteilung antennaler sensillen bei der honigbiene (Apis mellifera L.)."

[134] M. Hammer, "The neural basis of associative reward learning in honeybees," Trends Neurosci, vol. 20, no. 6, pp. 245-52, 1997.

[135] L. M. Kay and S. M. Sherman, "An argument for an olfactory thalamus," Trends Neurosci, vol. 30, no. 2, pp. 47-53, 2007.

[136] P. Duchamp-Viret, A. Duchamp, and M. A. Chaput, "Single olfactory sensory neurons simultaneously integrate the components of an odour mixture," Eur J Neurosci, vol. 18, no. 10, pp. 2690-6, 2003.

[137] N. Deisig, M. Giurfa, H. Lachnit, and J. C. Sandoz, "Neural representation of olfactory mixtures in the honeybee antennal lobe," Eur $J$ Neurosci, vol. 24, no. 4, pp. 1161-74, 2006.

[138] N. Deisig, M. Giurfa, and J. C. Sandoz, "Antennal lobe processing increases separability of odor mixture representations in the honeybee," J Neurophysiol, vol. 103, no. 4, pp. 2185-94, 2010.

[139] L. S. Kuebler, S. B. Olsson, R. Weniger, and B. S. Hansson, "Neuronal processing of complex mixtures establishes a unique odor representation in the moth antennal lobe," Front Neural Circuits, vol. 5, p. 7, 2011.

[140] R. Tabor, E. Yaksi, J. M. Weislogel, and R. W. Friedrich, "Processing of odor mixtures in the zebrafish olfactory bulb," J Neurosci, vol. 24, no. 29, pp. 6611-20, 2004.

[141] I. G. Davison and L. C. Katz, "Sparse and selective odor coding by mitral/tufted neurons in the main olfactory bulb," J Neurosci, vol. 27, no. 8, pp. 2091-101, 2007.

[142] J. P. Rospars, P. Lansky, M. Chaput, and P. Duchamp-Viret, "Competitive and noncompetitive odorant interactions in the early neural coding of odorant mixtures," J Neurosci, vol. 28, no. 10, pp. 2659-66, 2008.

[143] D. Münch, B. Schmeichel, A. F. Silbering, and C. G. Galizia, "Weaker ligands can dominate an odor blend due to syntopic interactions," Chem Senses, vol. 38, no. 4, pp. 293-304, 2013. 
[144] K. Shen, S. Tootoonian, and G. Laurent, "Encoding of mixtures in a simple olfactory system," Neuron, vol. 80, no. 5, pp. 1246-62, 2013.

[145] D. G. Laing and G. W. Francis, "The capacity of humans to identify odors in mixtures," Physiol Behav, vol. 46, no. 5, pp. 809-14, 1989.

[146] A. Livermore and D. G. Laing, "Influence of training and experience on the perception of multicomponent odor mixtures," J Exp Psychol Hum Percept Perform, vol. 22, no. 2, pp. 267-77, 1996.

[147] A. G. Khan, M. Thattai, and U. S. Bhalla, "Odor representations in the rat olfactory bulb change smoothly with morphing stimuli," Neuron, vol. 57 , no. 4, pp. 571-85, 2008.

[148] A. Meyer and C. G. Galizia, "Elemental and configural olfactory coding by antennal lobe neurons of the honeybee (Apis mellifera)," J Comp Physiol A Neuroethol Sens Neural Behav Physiol, vol. 198, no. 2, pp. 159-71, 2012.

[149] A. Capurro, F. Baroni, S. B. Olsson, L. S. Kuebler, S. Karout, B. S. Hansson, and T. C. Pearce, "Non-linear blend coding in the moth antennal lobe emerges from random glomerular networks," Front Neuroeng, vol. 5, p. 6, 2012.

[150] T. Niewalda, T. Voller, C. Eschbach, J. Ehmer, W. C. Chou, M. Timme, A. Fiala, and B. Gerber, "A combined perceptual, physico-chemical, and imaging approach to 'odour-distances' suggests a categorizing function of the Drosophila antennal lobe," PLoS One, vol. 6, no. 9, p. e24300, 2011.

[151] T. A. Cleland, "Early transformations in odor representation," Trends Neurosci, vol. 33, no. 3, pp. 130-9, 2010.

[152] C. Linster and T. A. Cleland, "Glomerular microcircuits in the olfactory bulb," Neural Netw, vol. 22, no. 8, pp. 1169-73, 2009.

[153] S. X. Luo, R. Axel, and L. F. Abbott, "Generating sparse and selective thirdorder responses in the olfactory system of the fly," Proc Natl Acad Sci U S A, vol. 107, no. 23, pp. 10713-8, 2010.

[154] M. T. Wiechert, B. Judkewitz, H. Riecke, and R. W. Friedrich, "Mechanisms of pattern decorrelation by recurrent neuronal circuits," Nat Neurosci, vol. 13, no. 8, pp. 1003-10, 2010.

[155] J. H. Proske, M. Wittmann, and C. G. Galizia, "Olfactory sensor processing in neural networks: lessons from modeling the fruit fly antennal lobe," Front Neuroeng, vol. 5, p. 2, 2012.

[156] T. Tetzlaff, M. Helias, G. T. Einevoll, and M. Diesmann, "Decorrelation of neural-network activity by inhibitory feedback," PLoS Comput Biol, vol. 8, no. 8, p. e1002596, 2012.

[157] A. A. Koulakov and D. Rinberg, "Sparse incomplete representations: a potential role of olfactory granule cells," Neuron, vol. 72, no. 1, pp. 124-36, 2011.

[158] K. Svoboda, W. Denk, D. Kleinfeld, and D. W. Tank, "In vivo dendritic calcium dynamics in neocortical pyramidal neurons," Nature, vol. 385, no. 6612, pp. 161-5, 1997.

[159] S. Charpak, J. Mertz, E. Beaurepaire, L. Moreaux, and K. Delaney, "Odorevoked calcium signals in dendrites of rat mitral cells," Proceedings of the National Academy of Sciences, vol. 98, no. 3, pp. 1230-1234, 2001. 
[160] C. G. Galizia and B. Kimmerle, "Physiological and morphological characterization of honeybee olfactory neurons combining electrophysiology, calcium imaging and confocal microscopy," J Comp Physiol A Neuroethol Sens Neural Behav Physiol, vol. 190, no. 1, pp. 21-38, 2004.

[161] W. Denk, J. H. Strickler, and W. W. Webb, "Two-photon laser scanning fluorescence microscopy," Science, vol. 248, no. 4951, pp. 73-6, 1990.

[162] M. C. Larsson, A. I. Domingos, W. D. Jones, M. E. Chiappe, H. Amrein, and L. B. Vosshall, "Or83b encodes a broadly expressed odorant receptor essential for Drosophila olfaction," Neuron, vol. 43, no. 5, pp. 703-14, 2004.

[163] R. I. Wilson, "Early olfactory processing in Drosophila: mechanisms and principles," Annu Rev Neurosci, vol. 36, pp. 217-41, 2013.

[164] R. Haddad, T. Weiss, R. Khan, B. Nadler, N. Mandairon, M. Bensafi, E. Schneidman, and N. Sobel, "Global features of neural activity in the olfactory system form a parallel code that predicts olfactory behavior and perception," J Neurosci, vol. 30, no. 27, pp. 9017-26, 2010.

[165] M. Nozawa and M. Nei, "Evolutionary dynamics of olfactory receptor genes in Drosophila species," Proc Natl Acad Sci U S A, vol. 104, no. 17, pp. 7122-7, 2007.

[166] K. Mori and Y. Yoshihara, "Molecular recognition and olfactory processing in the mammalian olfactory system," Prog Neurobiol, vol. 45, no. 6, pp. 585-619, 1995.

[167] O. Mazor and G. Laurent, "Transient dynamics versus fixed points in odor representations by locust antennal lobe projection neurons," Neuron, vol. 48, no. 4, pp. 661-73, 2005.

[168] K. Y. Chong, A. Capurro, S. Karout, and T. C. Pearce, "Stimulus and network dynamics collide in a ratiometric model of the antennal lobe macroglomerular complex," PLoS One, vol. 7, no. 1, p. e29602, 2012. 
